\title{
The Galactic WC stars
}

\section{Stellar parameters from spectral analyses indicate a new evolutionary sequence $^{\star}$}

\author{
A. Sander, W.-R. Hamann, and H. Todt
}

\begin{abstract}
Institut für Physik und Astronomie, Universität Potsdam, Karl-Liebknecht-Str. 24/25, 14476 Potsdam, Germany
e-mail: [ansander;wrh]@astro.physik.uni-potsdam.de
\end{abstract}

Received 5 August 2011 / Accepted 30 January 2012

\begin{abstract}
Context. The life cycles of massive stars from the main sequence to their explosion as supernovae or gamma ray bursts are not yet fully clear, and the empirical results from spectral analyses are partly in conflict with current evolutionary models. The spectral analysis of Wolf-Rayet stars requires the detailed modeling of expanding stellar atmospheres in non-LTE. The Galactic WN stars have been comprehensively analyzed with such models of the latest stage of sophistication, while a similarly comprehensive study of the Galactic WC sample remains undone.

Aims. We aim to establish the stellar parameters and mass-loss rates of the Galactic WC stars. These data provide the empirical basis of studies of (i) the role of WC stars in the evolution of massive stars, (ii) the wind-driving mechanisms, and (iii) the feedback of WC stars as input to models of the chemical and dynamical evolution of galaxies.

Methods. We analyze the nearly complete sample of un-obscured Galactic WC stars, using optical spectra as well as ultraviolet spectra when available. The observations are fitted with theoretical spectra, using the Potsdam Wolf-Rayet (PoWR) model atmosphere code. A large grid of line-blanked models has been established for the range of WC subtypes WC4 - WC8, and smaller grids for the WC9 parameter domain. Both WO stars and WN/WC transit types are also analyzed using special models.

Results. Stellar and atmospheric parameters are derived for more than 50 Galactic WC and two WO stars, covering almost the whole Galactic WC population as far as the stars are single, and un-obscured in the visual. In the Hertzsprung-Russell diagram, the WC stars reside between the hydrogen and the helium zero-age main sequences, having luminosities $L$ from $10^{4.9}$ to $10^{5.6} L_{\odot}$. The mass-loss rates scale very tightly with $L^{0.8}$. The two WO stars in our sample turn out to be outstandingly hot $(\approx 200 \mathrm{kK})$ and do not fit into the WC scheme.

Conclusions. By comparing the empirical WC positions in the Hertzsprung-Russell diagram with evolutionary models, and from recent supernova statistics, we conclude that WC stars have evolved from initial masses between 20 solar masses and $45 M_{\odot}$. In contrast to previous assumptions, it seems that WC stars in general do not descend from the most massive stars. Only the WO stars might stem from progenitors that have been initially more massive than $45 M_{\odot}$.
\end{abstract}

Key words. stars: massive - stars: mass-loss - stars: Wolf-Rayet - stars: evolution - stars: atmospheres - stars: winds, outflows

\section{Introduction}

Wolf-Rayet (WR) stars represent advanced evolutionary stages in the evolution of massive stars. Owing to their high temperatures and high mass-loss rates, WR stars provide a large amount of ionizing photons, mechanical momentum, and matter to their environment, making WR stars a keystone in the cosmic evolution. Because of the extreme non-local thermal equilibrium (non-LTE) situations in the expanding atmospheres, their spectral analysis only became possible after adequate, powerful and complex model atmosphere codes had been established. Since the early 90s, these tools have been available. Early analyses have been published, e.g., by Hillier (1987), Schmutz et al. (1989), Hamann et al. (1993), and Hamann et al. (1995) for the WN stars, i.e. WR stars with strong nitrogen emission. For the WC subclass, which are characterized by strong carbon emission-lines, wind models have been applied by, e.g., Hillier (1989), Hamann et al. (1992), and Koesterke et al. (1992).

The models have since been significantly improved. With the inclusion of iron line blanketing and microclumping (Hillier \& Miller 1999; Gräfener et al. 2002), a new generation of models

\footnotetext{
* Appendices A and B are available in electronic form at http://www. aanda.org
}

have allowed better spectral fits, and led to a revision of the empirical stellar parameters. A comprehensive re-analysis of the Galactic WN stars was performed by Hamann et al. (2006). Basically all WN stars in the Large Magellanic Cloud have been analyzed by Rühling (2008, and in prep.). Pasemann et al. (2011) studied the WR stars in the Small Magellanic Cloud. In contrast to the WN spectra, the WC spectra turned out to be much harder to reproduce. Our model spectra can fit most, but not all of the observed features, leaving some ambiguity about the choice of the best-fit model.

The present study is based on models that are not hydrodynamically consistent. The mass-loss rate and wind velocity field are instead treated as free parameters. The only hydrodynamical WC model that has been constructed so far is that developed to study the prototypical WC5 star WR 111 (Gräfener \& Hamann 2005). This model arrived at a much higher stellar temperature $(140 \mathrm{kK})$ and smaller mass-loss rate than the semi-empirical approach of the present paper. Using this same model, Gräfener et al. (2002) had obtained $T_{*}=85 \mathrm{kK}$ for WR 111, while only $62 \mathrm{kK}$ had been derived with non-blanketed models (Koesterke \& Hamann 1995).

In the afore mentioned paper of Koesterke \& Hamann (1995), 25 Galactic WC stars of subtypes WC4 to WC8 were 
analyzed with models containing only carbon and helium. After the inclusion of oxygen and the line-blanketing by iron-group elements, Barniske et al. (2006) re-analyzed a few of these WC stars and found that a carbon mass fraction of $40 \%$ appears to be typical.

The present study now covers for the first time a large part of the Galactic sample of WC stars of all subtypes. The analyses comprise 40 WC stars of subtypes WC4 to WC9. In addition, we consider six stars of the WN/WC transition type and two WO stars. The spectral analyses presented in this work are appropriate for single-star spectra. A few binaries are included in order to demonstrate the bias of the obtained parameters if the composite nature of their spectra is neglected.

The empirical stellar parameters and mass-loss rates of the Galactic WC stars established by our analyses provide the empirical basis for studies of (i) the role of WC stars in the evolution of massive stars, (ii) the wind-driving mechanisms, and (iii) the feedback of WC stars as input to the modeling of the chemical and dynamical evolution of galaxies.

In the next section, we introduce our sample of stars and the observational data used in this work. The model calculations are characterized in Sect. 3. In Sect. 4, we describe the analyses, and in Sect. 5 we present the results and comment on individual stars. Section 6 then discusses the results with regard to stellar evolution, before arriving at our conclusions in the final section (Sect. 7).

\section{Observational data}

The current WR catalog from van der Hucht $(2001,2006)$ lists 298 WR stars in our Galaxy, of which 113 are of WC type. However, most of the WR stars that have been added to the catalog since its previous release (van der Hucht 2001) were discovered in infrared observations, and suffer from high interstellar extinction in the visual range. Since our analyses are based on optical spectra, our sample is basically restricted to the list of van der Hucht (2001).

A significant fraction of the WR stars are known or suspected to have a binary companion, mostly of $\mathrm{O}$ type. Some of the stars analyzed by Koesterke \& Hamann (1995), for instance, have since been found to be binaries. In WR+O binaries, the spectrum is composed of both components. Often the O-star's contribution even dominates the overall spectrum. The decomposition of these spectra is beyond the scope of this paper. Nevertheless, we include a few of these composite spectra in our study and analyze them as if they were single WR stars, to illustrate the effects on the results.

A visible effect of an O-star companion is the so-called "diluted emission lines" (d.e.l.). When the light of a companion contributes significantly to the spectral continuum, the emission lines appear weaker in the normalized spectrum, hence are called "diluted". However, we do not a priori judge a WC star as a binary on the basis of apparently diluted emission lines alone. The proof of binarity can only be derived from radial-velocity variations or an eclipse light curve. The d.e.l. binary criterion is based on the assumption that stars of the same subtype have similarly strong emission lines. For WN stars, Hamann et al. (2006) demonstrated that this is not the case. However, we see below that the WC sequence has rather uniform spectra, making the d.e.l. criterion a valid binary indicator.

For a few putatively single, visually un-obscured WC stars, we have no spectra at our disposal, which leaves a sample of 56 stars for our study. In addition to the proper WC stars, our sample comprises two WO stars, five WN/WC transition types, and one WC/WN star (see Table 1).

For the analyses, we collected optical spectra from both Torres \& Massey (1987) and our own observations performed at the Calar Alto observatory or ESO. The optical spectra typically cover the wavelength range 3300-7300 $\AA$. In some cases however, if a star e.g. is not bright enough in a certain wavelength regime or there were technical difficulties during the observation, only a part of this range is covered. The typical spectral resolution is around $10 \AA$ (Torres \& Massey 1987). For the ultraviolet range, IUE spectra were retrieved from the INES archive. They cover the range from $1150 \AA$ to $3200 \AA$ with a typical resolution of around $6 \AA$. Infrared photometry $(J, H, K)$ was taken from the 2MASS catalog (Skrutskie et al. 2006), and in two cases (WR 52, WR 142) was augmented by Spitzer IRS observations. For some stars, we used additional optical narrowband photometry by Lundström \& Stenholm (1984), MSX infrared photometry (Egan et al. 2003), and/or Spitzer spectra from Ardila et al. (2010).

\section{Model grid}

To reproduce the observed spectra, we used the Potsdam WolfRayet (PoWR) code for expanding stellar atmospheres. The model basics are described in Gräfener et al. (2002) and Hamann \& Gräfener (2004). The non-LTE radiative transfer is calculated for a spherically symmetric and stationary outflow with a predescribed velocity field. In the supersonic part, we adopt the usual $\beta$-law with the terminal velocity $v_{\infty}$. The exponent $\beta$ is set to 1.0 throughout this study, except for the two WO stars for which $\beta=0.8$ gave slightly better fits. In the subsonic part, the velocity field is defined by the requirement that the density stratification approaches the hydrostatic limit, as described by Hamann \& Schmutz (1987). Since the mass-loss rate $\dot{M}$ and the velocity field are treated as free parameters, the models are not hydrodynamically consistent.

Wind inhomogeneities are taken into account in the "microclumping" approximation, assuming that individual "clumps" are optically thin at all frequencies, and that the interclump medium is void. The clumping factor $D$ denotes the density within the clumps, relative to the homogeneous model with the same $\dot{M}$, and is assumed to be constant throughout the atmosphere in this study.

Complex model atoms are taken into account, as summarized in Table 2. Iron-group elements are treated in the superlevel approximation (Gräfener et al. 2002).

The inner boundary of our model atmospheres is located at a radial optical depth of $\tau_{\text {Ross }}=20$, which we define to correspond to the stellar radius $R_{*}$. We define the "stellar temperature" $T_{*}$ as the effective temperature related to that radius, via StefanBoltzmann's law

$L=4 \pi R_{*}^{2} \sigma_{\mathrm{SB}} T_{*}^{4}$

and the stellar luminosity $L$.

As Schmutz et al. (1989) found out, stellar winds produce very similar emission-line spectra when they agree in a certain combination of the mass-loss rate and other parameters. To exploit this scaling invariance, they introduced the so-called "transformed radius"

$R_{\mathrm{t}}=R_{*}\left[\frac{v_{\infty}}{2500 \mathrm{~km} \mathrm{~s}^{-1}} / \frac{\dot{M} \sqrt{D}}{10^{-4} M_{\odot} / \mathrm{yr}}\right]^{\frac{2}{3}}$ 
Table 1. Galactic WC stars, not visually obscured (van der Hucht 2001).

\begin{tabular}{|c|c|c|c|c|c|c|c|}
\hline WR & Subtype $^{1}$ & Figure $^{2}$ & Remarks $^{3}$ & WR & Subtype $^{1}$ & Figure $^{2}$ & Remarks $^{3}$ \\
\hline 4 & WC5 & B.1 & & & & & \\
\hline 5 & WC6 & B. 2 & & $75 \mathrm{a}$ & WC9 & - & no spectra \\
\hline $7 \mathrm{a}$ & WN4h/WC & - & no spectra & 76 & WC9d & - & no spectra \\
\hline 8 & WN7/WCE & B. 3 & binary $\mathrm{WN}+\mathrm{WC}$ ? & 77 & $\mathrm{WC} 8+\mathrm{OB}$ & - & binary \\
\hline 9 & $\mathrm{WC5}+\mathrm{O} 7$ & - & binary & 79 & $\mathrm{WC} 7+\mathrm{O} 5-8$ & - & binary \\
\hline 11 & $\mathrm{WC} 8+07.5$ & - & binary & 80 & WC9d & B. 27 & \\
\hline 13 & WC7 & B. 4 & & 81 & WC9 & B. 28 & \\
\hline 14 & $\mathrm{WC7}+?$ & B. 5 & binary? no d.e.l. & 86 & WC7+B0I-III & B. 29 & binary: pseudo fit $^{5}$ \\
\hline 15 & WC6 & B.6 & & 88 & WC9+? & B. 30 & $\mathrm{WN}+\mathrm{WC}$ or $\mathrm{WN} / \mathrm{WC}^{6}$ \\
\hline 17 & WC5 & B.7 & & 90 & WC7 & B. 31 & \\
\hline 19 & WC4pd+09.6 & - & binary & 92 & WC9 & B. 32 & \\
\hline $20 \mathrm{a}$ & WN7:h/WC & - & now WN6ha+WN6ha ${ }^{4}$ & 93 & $\mathrm{WC} 7+\mathrm{O} 7-9$ & - & binary \\
\hline 23 & WC6 & B. 8 & & 95 & WC9d & B. 33 & \\
\hline 26 & WN7/WCE & B.9 & & 96 & WC9d & - & no spectra \\
\hline 27 & WC6 & B. 10 & & 98 & WN8/WC7 & B. 34 & now WN7/WC+O8-97 : pseudo fit \\
\hline 30 & WC6+O6-8 & - & binary & $98 \mathrm{a}$ & WC8-9vd+? & - & no spectra \\
\hline $30 \mathrm{a}$ & WO4+O5-5.5 & - & binary & 101 & WC8 & - & no spectra \\
\hline $31 \mathrm{c}$ & WC6+OB & - & binary & 102 & WO2 & B. 35 & \\
\hline 32 & $\mathrm{WC} 5+\mathrm{OB}$ & - & binary & 103 & WC9d & B. 36 & \\
\hline 33 & WC5 & B. 11 & & 104 & $\mathrm{WC} 9 \mathrm{~d}+\mathrm{B} 0.5 \mathrm{~V}$ & B. 37 & binary: pseudo fit ${ }^{5}$ \\
\hline 38 & WC4 & B. 12 & binary? & 106 & WC9d & B. 38 & \\
\hline $38 b$ & $\mathrm{WC} 7+\mathrm{OB}$ & - & binary & $107 \mathrm{a}$ & WC6 & - & no spectra \\
\hline 39 & $\mathrm{WC} 7+\mathrm{OB}$ & B. 13 & binary: pseudo $\mathrm{fit}^{5}$ & 111 & WC5 & B. 39 & \\
\hline 41 & $\mathrm{WC} 5+\mathrm{OB}$ & - & binary & 112 & WC9d+OB & - & binary \\
\hline 42 & $\mathrm{WC} 7+\mathrm{O} 7 \mathrm{~V}$ & - & binary & 113 & WC8d+O8-9IV & B. 40 & binary: pseudo fit ${ }^{5}$ \\
\hline 45 & WC6 & B. 14 & & 114 & WC5 & B. 41 & \\
\hline $47 \mathrm{c}$ & WC5 & - & no spectra & 117 & WC9d & B. 42 & \\
\hline 48 & WC6+09.5 & - & binary & 118 & WC9d & - & no spectra \\
\hline $48 \mathrm{a}$ & WC9d & - & no spectra & 119 & WC9d & B. 43 & \\
\hline $48 b$ & WC8ed & - & no spectra & 121 & WC9d & B. 44 & \\
\hline 50 & $\mathrm{WC} 7+\mathrm{OB}$ & B. 15 & binary: pseudo fit ${ }^{5}$ & 125 & WC7ed+O9III & B. 45 & binary: pseudo fit ${ }^{5}$ \\
\hline 52 & WC4 & B. 16 & & 126 & WC5/WN & B. 46 & \\
\hline 53 & WC8d & B. 17 & & 132 & WC6 & B. 47 & \\
\hline 56 & WC7 & B. 18 & & 135 & WC8 & B. 48 & \\
\hline 57 & WC8 & B. 19 & & 137 & WC7pd+O9 & B. 49 & binary: pseudo fit ${ }^{5}$ \\
\hline 58 & WN4/WCE & B. 20 & & 140 & $\mathrm{WC7pd}+\mathrm{O} 4-5$ & - & binary \\
\hline 59 & $\mathrm{WC} 9 \mathrm{~d}+\mathrm{OB} ?$ & B. 21 & binary? & 142 & WO2 & B. 50 & \\
\hline 60 & WC8 & B. 22 & & 143 & $\mathrm{WC} 4+\mathrm{Be}$ & B. 51 & binary: pseudo fit ${ }^{5}$ \\
\hline 64 & WC7 & B. 23 & & 144 & WC4 & B. 52 & \\
\hline 65 & $\mathrm{WC} 9 \mathrm{~d}+\mathrm{OB} ?$ & B. 24 & binary? & 145 & WN7/WCE & B. 53 & \\
\hline 68 & WC7 & B. 25 & & 146 & WC6+O8 & B. 54 & binary: pseudo fit $^{5}$ \\
\hline 69 & WC9d+OB? & B. 26 & binary? & 150 & WC5 & B. 55 & \\
\hline 70 & WC9vd+B0I & - & binary & $153 \mathrm{ab}$ & WN6/WCE+O6I & - & binary \\
\hline 73 & WC9d & - & no spectra & 154 & WC6 & B.56 & \\
\hline
\end{tabular}

Notes. ${ }^{(1)}$ Classification from van der Hucht (2001). ${ }^{(2)}$ Spectral fits, shown in the appendix. ${ }^{(3)}$ Further comments to the individual stars are given in Sect. A. ${ }^{(4)}$ Nazé et al. (2008). ${ }^{(5)}$ Known binary fitted as if it were a single star (as discussed in Sect. 2). ${ }^{(6)}$ Williams et al. (2005). ${ }^{(7)}$ Gamen \& Niemela (2003).

(the clumping factor $D$ has been incorporated in this definition by Hamann \& Koesterke 1998).

The name "transformed radius", historically coined by Schmutz et al. (1989), is actually misleading since $R_{\mathrm{t}}$ does not have the meaning (although the units) of a radius. A more appropriate quantity to consider would be $R_{\mathrm{t}}^{-3}$, which might be called a "normalized emission measure". Since this quantity is proportional to the volume integral of the density squared, divided by the stellar surface, $R_{\mathrm{t}}^{-3}$ scales with the emission from recombination lines normalized to the continuum. This explains why different combinations of $R_{*}, v_{\infty}$, and mass-loss rate $\dot{M}$ result in approximately the same normalized WR emission-line strengths as long as $R_{\mathrm{t}}\left(\right.$ or $\left.R_{\mathrm{t}}^{-3}\right)$ is kept at the same value.

Wolf-Rayet model spectra are most sensitive to $T_{*}$ and $R_{\mathrm{t}}$. When finding systematically the model that provides the closest fit to a given observation, it is therefore most convenient to rely on model grids in which these two parameters are varied. The other parameters, namely the chemical composition and the terminal wind velocity, are kept constant within one model grid.

We established one large model grid for most of the WC parameter space, and two smaller grids for the WC9 domain. The grid resolution is 0.05 in $\log T_{*}$ and 0.1 in $\log R_{\mathrm{t}}$. For the chemical composition, we assumed mass fractions of $55 \%$ helium, $40 \%$ carbon, $5 \%$ oxygen, and $0.16 \%$ iron-group elements (cf. Table 2). To verify that these abundances are adequate to the observed stars, we also calculated smaller grids with $60 \%$ and $20 \%$ carbon fractions, respectively.

For the main WC grid, we set the terminal wind velocity to $2000 \mathrm{~km} \mathrm{~s}^{-1}$, which is in the middle of the range actually observed. The two WC9 grids are calculated for $1000 \mathrm{~km} \mathrm{~s}^{-1}$ and 
$1600 \mathrm{~km} \mathrm{~s}^{-1}$. Owing to their their lower ionization, the WC9 models include an extended $\mathrm{C}_{\text {II }}$ model atom.

Throughout this study, the microturbulence velocity is set to $100 \mathrm{~km} \mathrm{~s}^{-1}$, based on the experience that this yields profile shapes similar to the observed ones. For the clumping density factor, we adopt $D=10$, which generally reproduces the observed strength of the electron-scattering wings. However, the true degree of the wind inhomogeneity remains the subject of debate (cf. Hamann et al. 2008). According to Eq. (2), the empirically obtained mass-loss rates scale with the adopted clumping factor as $\dot{M} \propto D^{-1 / 2}$.

The luminosity was kept fixed over all our grids at $\log L / L_{\odot}=5.3$. According to Eqs. (1) and (2), the scaling of the model to a different luminosity implies a scaling of the mass-loss rate as $\dot{M} \propto L^{3 / 4}$. In a strict sense, the scaling invariance between models of the same $R_{\mathrm{t}}$ is only approximate, but accurate enough to cover the range of luminosities in our sample.

Figures 1 and 2 display contour plots for the strength of the brightest WC emission lines. The small dots represent the calculated models of the standard WC grid. Each contour refers to a certain equivalent width, labeled with $-W_{\lambda}$ in $\AA$. The ratio of the lines $\mathrm{C}_{\text {III }} 5696 \AA$ to $\mathrm{C}_{\mathrm{IV}} 5808 \AA$ is used as the primary criterion for the WC subtype classification (Crowther et al. 1998). For early subtypes, O v $5590 \AA$ is also considered (van der Hucht 2001).

For the first time, we apply the PoWR code when analyzing spectra of the late subtype WC9. Most of these stars show an excess of infrared emission that is attributed to circumstellar dust, as denoted by the letter "d" appended to their classification, WC9d. This circumstellar dust usually leads to strong reddening of the stellar radiation, inhibiting their observation in the UV. The optical spectrum, however, does not suffer much from the dust emission and absorption, and can be analyzed with the help of our models together with the non-dusty WC9 stars.

The WC9 stars have a lower stellar temperature than the earlier WC subtypes, which is evident from the lower ions that are visible in their spectra. We therefore calculated special, small grids of models in the WC9 parameter domain, including here the extended $\mathrm{C}$ II model atom. Compared to the earlier subtypes, the WC9 stars have slower winds, as indicated by the smaller width of their lines. We therefore calculated one WC9 grid with $v_{\infty}=1600 \mathrm{~km} \mathrm{~s}^{-1}$ and another with only $v_{\infty}=1000 \mathrm{~km} \mathrm{~s}^{-1}$.

The two WO-type stars in our sample turned out to be very hot $\left(T_{*} \approx 200 \mathrm{kK}\right)$. We therefore had to include higher ions of the iron-group elements, up to Fe xv, in their models. The extremely broad emission lines indicate wind velocities as high as $v_{\infty} \approx 5000 \mathrm{~km} \mathrm{~s}^{-1}$, which is also much higher than for the WC stars. Hence, a special set of model calculations hat to be used to analyze the WO-type stars.

The synthetic spectra of the main WC grid and the WC9 subgrids will be made available from our website ${ }^{1}$ simultaneously with the release of this paper.

\section{The analysis method}

The stars are analyzed by finding the PoWR model that most closely fits the observations. The first part of the fit procedure refers to the normalized line spectrum, and the second part to the fit of the spectral energy distribution (SED). Since WC spectra are full of lines, a rectification "by eye" can be ambiguous. Fortunately, most of the spectra we used are calibrated in absolute fluxes. We therefore rectify the spectra by dividing them by

\footnotetext{
${ }^{1}$ http://www . astro.physik. uni-potsdam.de/PoWR.html
}

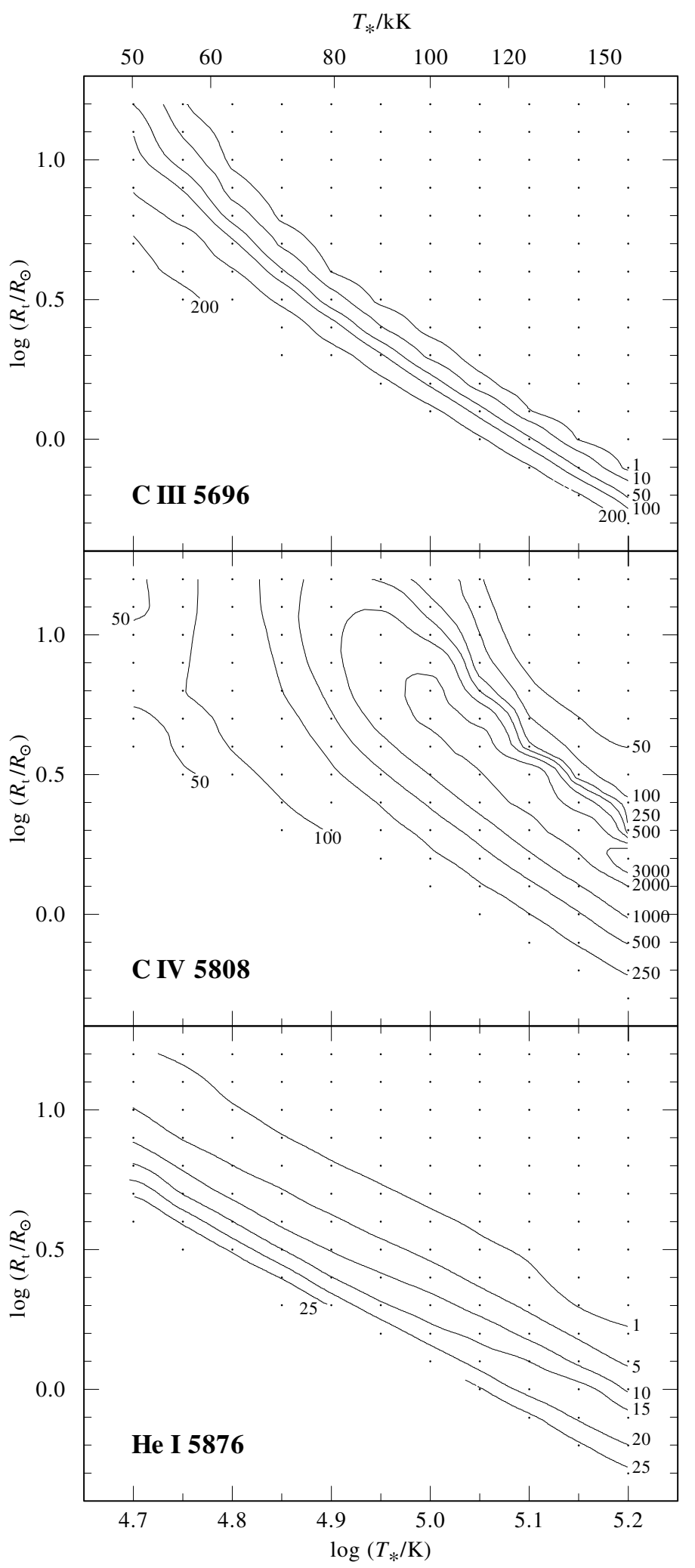

Fig. 1. Contours of constant equivalent widths of the WC emission lines $\mathrm{C}_{\text {III }} 5696 \AA \AA^{2}, \mathrm{C}_{\text {IV }} 5808 \AA$ and He I $5876 \AA$. The small dots represent models of the WC grid. Labels give absolute value of the equivalent width in $\AA$.

the model continuum. Consequently, the two steps (a) line fit and (b) SED fit are actually coupled and have to be iterated.

The WC stars are divided into subtypes based on the ratios of the equivalent widths of certain emission lines. The 


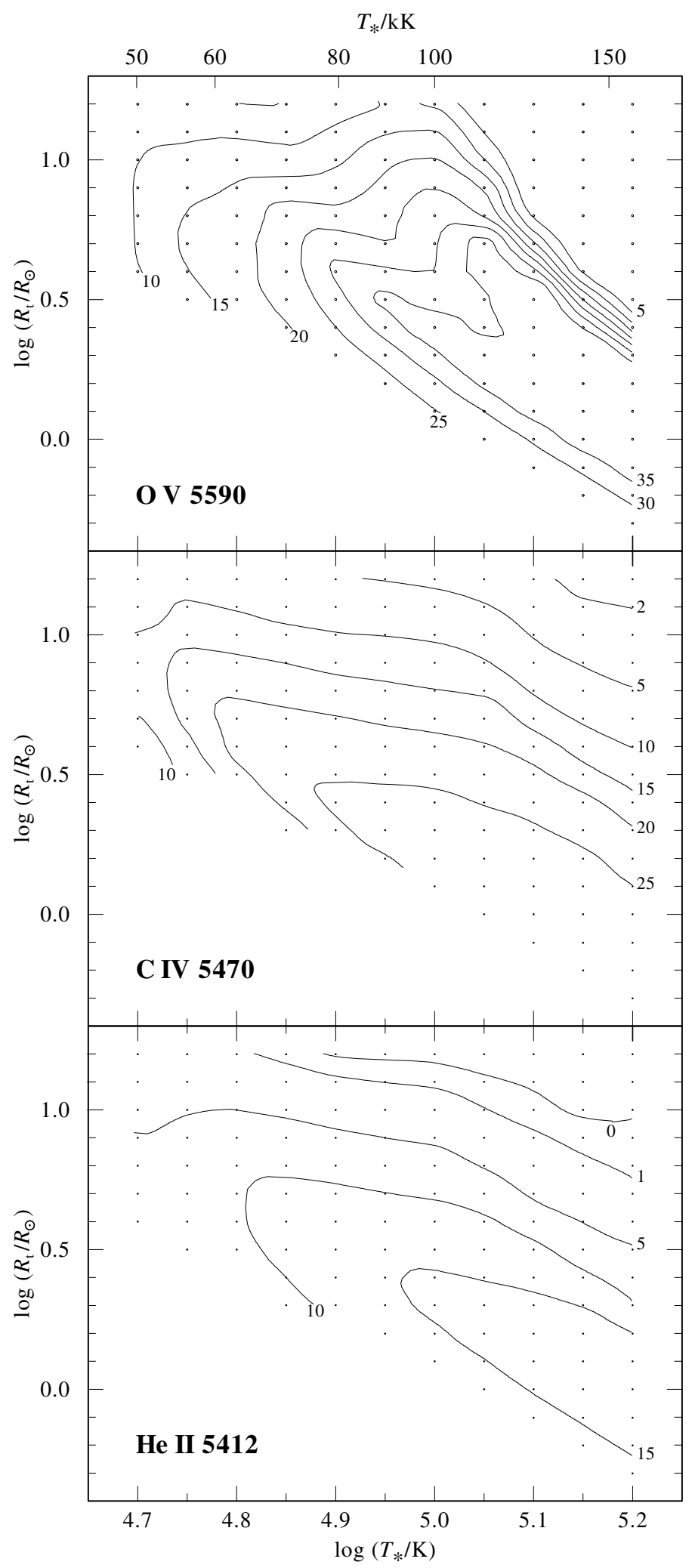

Fig. 2. Same as Fig. 1, but for the lines Ov $5590 \AA, C_{\text {Iv }} 5470 \AA$ and He II $5412 \AA$.

standard classification system was summarized by van der Hucht (2001) and uses the ratio of $C_{\text {IV }}$ to $C_{\text {III }}$ as a primary criterion. The main classification lines are C IV $5808 \AA$ and $C_{\text {III }} 5696 \AA$, where $\mathrm{C}_{\text {IV }} 5808 \AA$ is either one of two lines, together with the $\mathrm{C}$ III/C IV/He II-blend around $4650 \AA$, that are extremely strong in the optical spectrum of early WC subtypes.
Table 2. Model grid parameters.

\begin{tabular}{|c|c|c|}
\hline & WC grid & WC9 grids \\
\hline$X_{\mathrm{He}}$ & \multicolumn{2}{|r|}{$55 \%$} \\
\hline$X_{\mathrm{C}}$ & \multicolumn{2}{|r|}{$40 \%$} \\
\hline$X_{\mathrm{O}}$ & \multicolumn{2}{|c|}{$5 \%$} \\
\hline$X_{\mathrm{Fe}}{ }^{a}$ & \multicolumn{2}{|c|}{$0.16 \%$} \\
\hline $\log L / L_{\odot}$ & \multicolumn{2}{|r|}{5.3} \\
\hline $\begin{array}{l}v_{\infty} / \mathrm{km} \mathrm{s}^{-1} \\
D\end{array}$ & \multicolumn{2}{|r|}{10} \\
\hline \multicolumn{3}{|c|}{ Number of Levels } \\
\hline $\mathrm{He}_{\mathrm{I}}$ & & 17 \\
\hline He II & & 16 \\
\hline Не ІІІ & \multicolumn{2}{|r|}{1} \\
\hline $\mathrm{C}_{\mathrm{II}}$ & 3 & 29 \\
\hline $\mathrm{C}_{\text {III }}$ & \multicolumn{2}{|r|}{40} \\
\hline Civ & \multicolumn{2}{|r|}{19} \\
\hline $\mathrm{Cv}$ & \multicolumn{2}{|r|}{1} \\
\hline $\mathrm{O}_{\text {II }}$ & \multicolumn{2}{|r|}{3} \\
\hline $\mathrm{O}_{\text {III }}$ & \multicolumn{2}{|r|}{33} \\
\hline O IV & \multicolumn{2}{|r|}{25} \\
\hline $\mathrm{Ov}$ & \multicolumn{2}{|r|}{36} \\
\hline $\mathrm{O}_{\text {VI }}$ & \multicolumn{2}{|r|}{15} \\
\hline $\mathrm{O}_{\text {VII }}$ & \multicolumn{2}{|r|}{1} \\
\hline $\mathrm{Fe}$ III- $\mathrm{X}^{a}$ & \multicolumn{2}{|c|}{72 superlevels } \\
\hline
\end{tabular}

Notes. ${ }^{(a)}$ Generic element, representing also Sc, Ti, V, Cr, Mn, Co, and $\mathrm{Ni}$, with relative abundances to $\mathrm{Fe}$ as described in Gräfener et al. (2002). (b) We calculated two WC9 grids with different velocities.

One of our criteria for choosing the best-fit model is to ensure in particular that we can fit these strongest lines. However, the enormous peak heights of in particular the earlier subtypes can only be reached in the models at the price that other emission lines become stronger than observed. Thus, we often had to make some compromise with respect to the overall fit quality, and also paid attention to other significant lines such as Ov $5590 \AA$, the "diagnostic line pair" (He II $5412 \AA$ and $\mathrm{C}_{\text {IV }} 5470 \AA$ ), and the carbon lines in the UV. For some stars in our sample, the red part of the optical spectrum was not available, and our fit was restricted to the $4650 \AA$-blend and neighboring lines such as C IV $4441 \AA$ and sometimes O IV $3412 \AA$.

Hence, the line fit yields the stellar temperature $T_{*}$ and the "transformed radius" $R_{\mathrm{t}}$ as main parameters, together with constraints on the terminal wind velocity $v_{\infty}$ and the chemical composition. Figure 3 shows as an example the observed optical spectrum of the WC6 star WR 13, together with the synthetic spectrum from the best-fit model.

The PoWR models do not only provide a normalized spectrum but also the absolute flux values of a star that can be used to fit the SED if flux-calibrated spectra and/or photometric values are available. The dilution of the model flux depends on the distance and the reddening. For stars with a known distance modulus (D.M.), we can obtain the absolute magnitude $M_{v}$ by

$M_{v}=m_{v}-$ D.M. $-A_{v}$.

We note that the color indices ( $v$ and $b$ ) refer to the monochromatic magnitudes defined by Smith (1968). The value of $A_{v}$ is provided by the SED fit where the reddening parameter $E_{b-v}$ is a free parameter, as well as the detailed reddening law. Whenever adequate, we applied the reddening law from Seaton (1979), which implies a fixed ratio of extinction $A_{V}$ to color excess $E_{B-V}$ of $R_{V}=3.1$. If this turned out to be insufficient in reproducing 

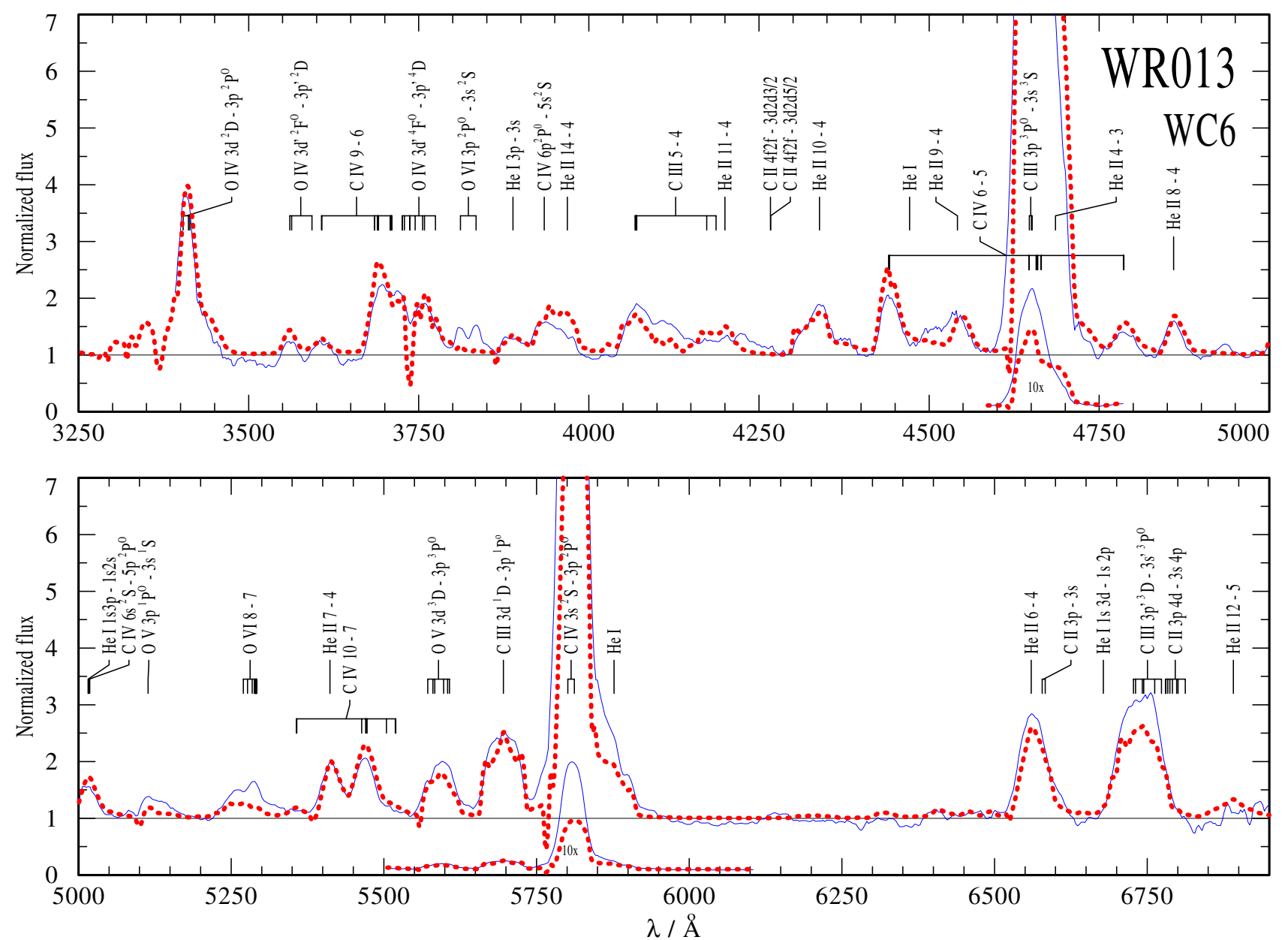

Fig. 3. Optical spectrum of the WC6 star WR 13. The solid thin line is the observed spectrum, the thick dotted line represents the best-fit WC grid model. The primary model parameters are $T_{*}=79.4 \mathrm{kK}$ and $\log R_{\mathrm{t}} / R_{\odot}=0.5$. The same model fits to most of the Galactic WC6 single stars, although it tends to underestimate the peak heights of $\mathrm{C}_{\mathrm{IV}} 5808 \AA$ and the $\mathrm{C}$ III/ $\mathrm{C}$ IV blend around $4650 \AA$.

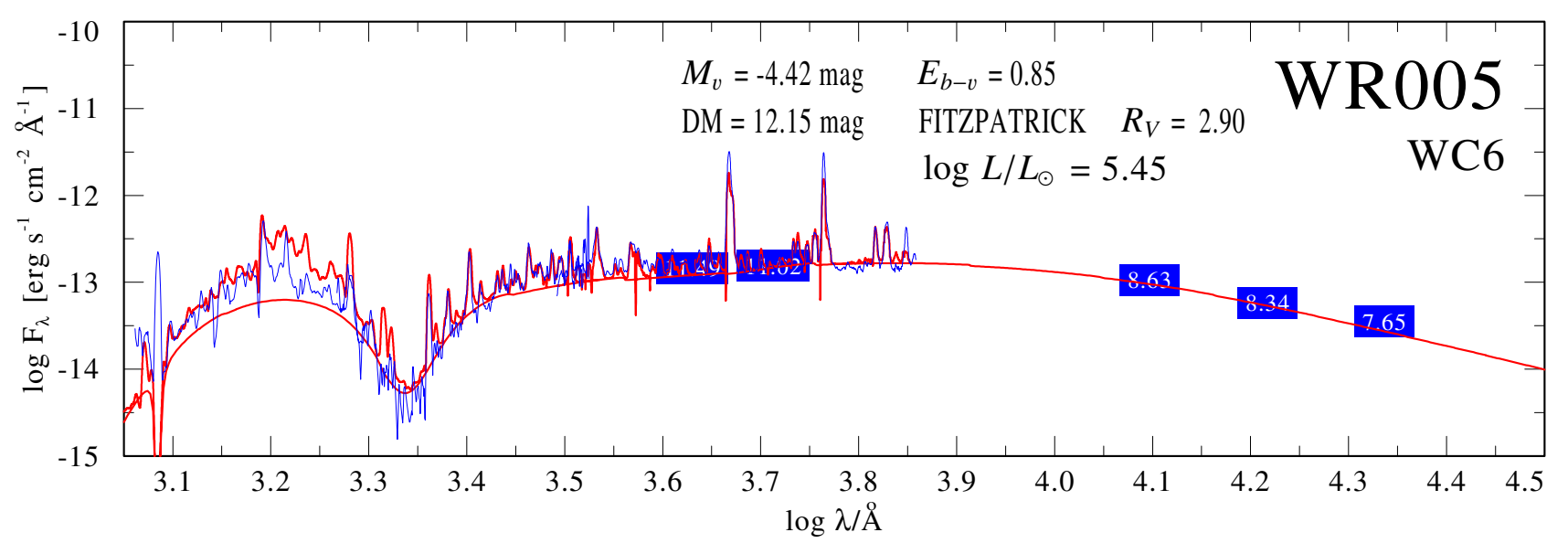

Fig. 4. Spectral energy distribution (SED) for the WC6 star WR 5. Flux-calibrated observations (blue thin and noisy line) are from IUE in the UV, Torres \& Massey (1987) in the optical, and 2MASS $(J, H$, and $K$ photometry) in the near-infrared. The synthetic spectrum is plotted as a red thick line. The continuum-only model flux is also shown for comparison. The model that fits the normalized line spectrum best has been selected from the WC standard grid $\left(T_{*}=79.4 \mathrm{kK}, \log R_{\mathrm{t}} / R_{\odot}=0.5\right)$.

the SED, we used the laws of Cardelli et al. (1989) or Fitzpatrick (1999), which treat $R_{V}$ as a free parameter, and adjusted the latter to optimize the SED fit. (The reddening law and $R_{V}$ are both indicated in Appendix B plots.) If mid-IR spectra were available for the SED fit, we always chose the reddening law of Fitzpatrick (1999).
An example SED fit is shown in Fig. 4. As the distance for this star is not known, an absolute magnitude $M_{v}=-4.42 \mathrm{mag}$ was adopted from our subtype calibration. With the help of Fig. 4, we found that the luminosity of the grid model must be scaled to $\log L / L_{\odot}=5.45$ in order to fit the observed SED. Simultaneously, the color excess $E_{b-v}$ is also properly adjusted, 
Table 3. Calibration of absolute magnitudes for the different WC subtypes, as derived from stars with distances known from their cluster or association membership (cf. Fig. 5).

\begin{tabular}{lc}
\hline \hline Subtype & $M_{v}[\mathrm{mag}]$ \\
\hline WC4 & -3.34 \\
WC5 & -4.12 \\
WC6 & -4.42 \\
WC7 & -4.18 \\
WC8 & -4.48 \\
WC9 & -5.13 \\
WN4/WCE & $-3.84^{a}$ \\
WN7/WCE & $-5.67^{a}$ \\
\hline
\end{tabular}

Notes. ${ }^{(a)}$ Adopted WN calibration from Hamann et al. (2006).

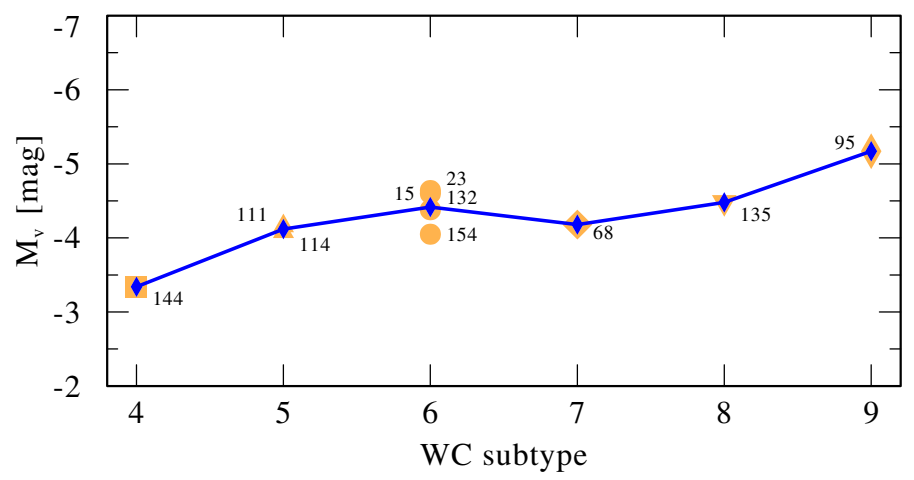

Fig. 5. $M_{v}$ calibration for the different WC subtypes, using stars for which the distance is known from their membership to an open cluster or association (labels: WR number). The symbols connected by the dark line show the calculated average values for $M_{v}$. The obtained values are listed in Table 3.

together with the choice of the reddening law ("CARDELLI") and its parameter $R_{V}$. The fit implies a distance modulus of D.M. = $12.15 \mathrm{mag}$.

The color excess $E_{b-v}$ in the Smith system is related to the more common $E_{B-V}$ value from the Johnson system by $E_{B-V}=$ $1.21 E_{b-v}$, while the extinction $A_{v}$ is given by

$A_{v}=\left(1.21 R_{V}+0.36\right) E_{b-v}$

as described by Lundström \& Stenholm (1984).

In general, it is hard to determine the distances of individual stars in our Galaxy, hence only for a subset of our sample are the distances known based on their membership to open clusters or associations (see Appendix A). For all other stars we follow the classical approach of assuming that all stars of the same subtype have the same absolute visual brightness $M_{v}$.

From the WC stars of our sample with known distances, the $M_{v}$ values are shown in Fig. 5. The average relation is also indicated and tabulated in Table 3 . It is unclear whether the assumption of a unique absolute brightness within each subtype is justified. The scatter in the $M_{v}$ values within one subtype in Fig. 5 may only reflect the errors in the adopted stellar distances, but could also by partly intrinsic to the stars. Within these uncertainties, the resulting $M_{v}$ values increase towards later subtypes. This is similar to the corresponding subtype calibration of the hydrogen-free WN stars (Hamann et al. 2006). We note that even the same visual brightness would still imply a sharp dependence of the luminosity on subtype, because of their different bolometric corrections (see below).
For the two WN/WC stars without known distances, we adopt the $M_{v}$ calibration for the corresponding hydrogen-free WN subtype of Hamann et al. (2006), because their chemical composition is more similar to WN than WC stars.

\section{Results}

\subsection{Stellar parameters}

The results of the analyses are compiled in Table 4 for our whole sample, now sorted by spectral subtype. The spectral fits and comments on individual stars are given in the appendix (see Appendices B and A).

We begin by discussing the results from the line fit, chiefly the stellar temperature and the transformed radius. Figure 6 shows the locations of the analyzed stars in the $\log R_{\mathrm{t}}-\log T_{*^{-}}$ plane. The striking result visible in that diagram is the clear, one-dimensional sequence of the WC subtypes. They are aligned along the linear relation

$\log R_{\mathrm{t}} \propto-2 \log T_{*}$

with little scatter. The stellar temperature of the WC stars correlates tightly with their subtype. The two WO stars in our sample, however, do not appear to follow an extrapolation of the WC sequence, but are much hotter.

Another group of points in Fig. 6 refers to stars that are known or highly suspected to be binaries with a luminous companion. We analyzed their spectra as if they were single stars. The parameters obtained by these "pseudo fits" clearly distinguish the binaries from the sequence of single WC stars. This independently confirms the composite nature of their spectra. Thus, the "diluted emission line" (d.e.l.) criterion, which is insufficient for WN stars as emphasized by Hamann et al. (2006), seems to work fine for WC stars when distinguishing single stars from binaries. We conclude that those stars that follow the WC sequence in Fig. 6 do not suffer significantly from the line dilution effect, i.e. they do not have a luminous companion.

The tight correlation of the parameters with WC subtype visible in Fig. 6 is now much clearer than in the first comprehensive analyses of Galactic WC stars by our group (Koesterke \& Hamann 1995). Moreover, the subclass of weak-lined WC stars (WC-w) that we had introduced in the old paper is no longer necessary: all WC-w stars have since been identified as binaries.

Some deviation from the straight alignment of subtypes is seen in Fig. 6 for the WC9 stars. The WC9 stars are characterized by the appearance of strong $\mathrm{C}$ II-lines, and are significantly cooler than the other WC subtypes.

In most of the WC9 spectra, the lines are narrower than in the simulations of our standard WC grid, which is calculated with a terminal wind speed of $v_{\infty}=2000 \mathrm{~km} \mathrm{~s}^{-1}$. We therefore calculated special grids in this parameter range with $v_{\infty}=1600 \mathrm{~km} \mathrm{~s}^{-1}$ and $v_{\infty}=1000 \mathrm{~km} \mathrm{~s}^{-1}$.

The fit quality for the WC9 spectra differs from those of the other subtypes. While the "IUE long" UV spectra (1900$3300 \AA$ ) are usually accurately reproduced and the "IUE short" range (1200-2000 $\AA$ ) is hardly met, the opposite is often true for the few WC9 stars with available UV spectra (WR 69, WR 92, WR 103).

In the optical part of the spectrum, most lines are reproduced consistently by our models, although the peak heights of C III $4648 \AA$ and some lines between $6500 \AA$ and $7200 \AA$ are not recreated. The temperature steps in our grid are a bit too coarse to find the optimum fit in some cases, e.g. when the lines 


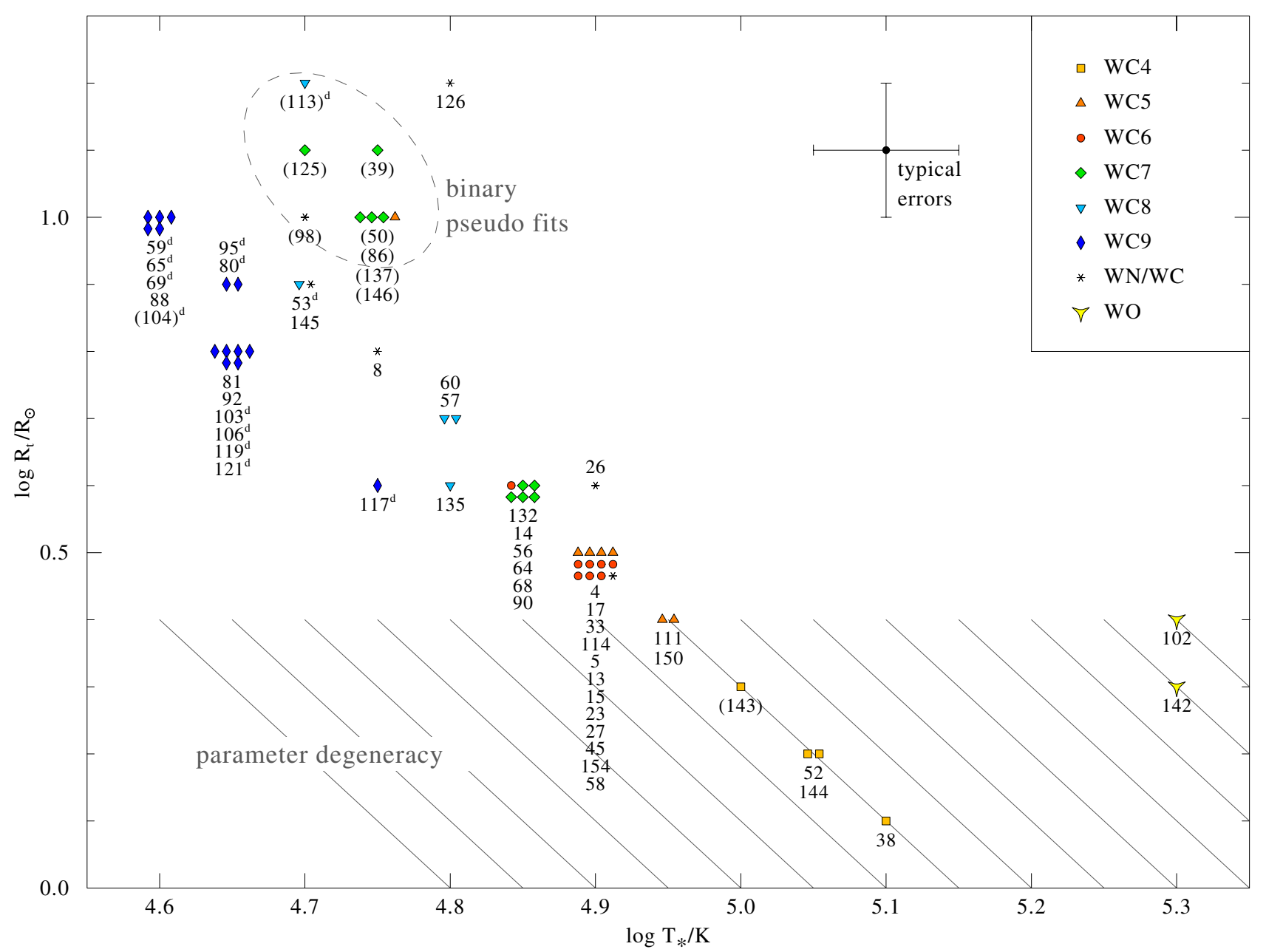

Fig. 6. The results of the WC analyses in the $\log T_{*}-\log R_{\mathrm{t}}$-plane, which corresponds to the basic model parameters. The WC stars form a onedimensional sequence with a slight offset for the WC9 stars. Stars appearing above the sequence turn out to be binaries. The symbol shapes indicate the different WC subtypes. The numbers next to the symbols are the WR numbers according to van der Hucht (2001). Binary systems analyzed as a pseudo fit have WR numbers in brackets. Stars with persistent dust emission are marked with a superscript "d". The thin grey lines in the lower part indicate the thick wind regime where stars can be shifted along these lines without significant changes in the synthetic spectrum.

C Iv $5808 \AA$ and He I $5876 \AA$ are observed with almost the same peak heights.

The WC9 stars of our sample cluster into two distinct groups in the $\log R_{\mathrm{t}}-\log T_{*}$-plane, one cooler group around $T_{*} \approx 40 \mathrm{kK}$ and $\log R_{\mathrm{t}}=1.0$, which we refer to as group $\mathrm{I}$, and another one with higher $T_{*} \approx 45 \mathrm{kK}$ and a denser wind $\left(\log R_{\mathrm{t}}=0.8\right)$, which we call group II. Could it be that group I consists of yet undetected binaries, while only the stars in group II are really single stars?

In group I, only WR 104 (the "pinwheel") is an established binary, but all others are suspected to be binaries (see appendix Sect. A for corresponding details about WR 59, WR 65, WR 69, and WR 88). On the other hand, the WC9d star WR 119 from the hotter group II was checked for binarity by Williams et al. (2005) with a negative result. The same holds for WR 117, which is even hotter than both groups and closer to the WC8 stars in Fig. 6. Two of the three WC9 stars in our sample that do not show dust (WR 81, and WR 92) belong to the hotter group II, which seem to consist of the definitive non-binaries. The third one (WR 88) seems to be a special case as discussed in Sect. A.

For very dense winds $\left(\log R_{\mathrm{t}} / R_{\odot} \lesssim 0.4\right)$, the spectra no longer depend on the two independent parameters $T_{*}$ and $R_{\mathrm{t}}$, but only on the product $R_{\mathrm{t}} T_{*}^{2}$ (Hamann et al. 2003). The physical reason for this approximate parameter degeneracy is that in very dense winds, all radiation (including the continuum) emerges from layers that expand with nearly the terminal wind speed. Hence, under these conditions, the mass-loss rate is the only relevant parameter when comparing models with same luminosity and $v_{\infty}$. Lines of constant $R_{\mathrm{t}} T_{*}^{2}$ (or, equivalently, constant $L / \dot{M}^{4 / 3}$ ) are indicated by thin parallel lines in the lower part of Fig. 6. The model spectra in this range are nearly the same along these lines, hence the specific combination of $R_{\mathrm{t}}$ and $T_{*}^{2}$ is not well-constrained. In other words, we see only rapidly moving layers of the atmosphere, while the slower part of the wind is opaque at all wavelengths, and therefore the radius of the hydrostatic stellar core is not observable. This degeneracy primarily affects the WC4 stars.

When available and not in conflict with our spectral fits, terminal velocities compiled in Table 4 were collected from Prinja et al. (1990) and Niedzielski \& Skorzynski (2002). These values had been derived from UV spectra, using the blue end of P Cygni line profiles. Therefore, they might differ from the terminal velocities obtained from optical emission lines. In these cases and for all remaining stars - mainly those without existing UV observations $-v_{\infty}$ was roughly estimated by ourselves from the widths of the optical lines. We note that the models used for the line fits shown in the Appendix are not calculated with the individual wind velocity of the star, but are taken from the models 


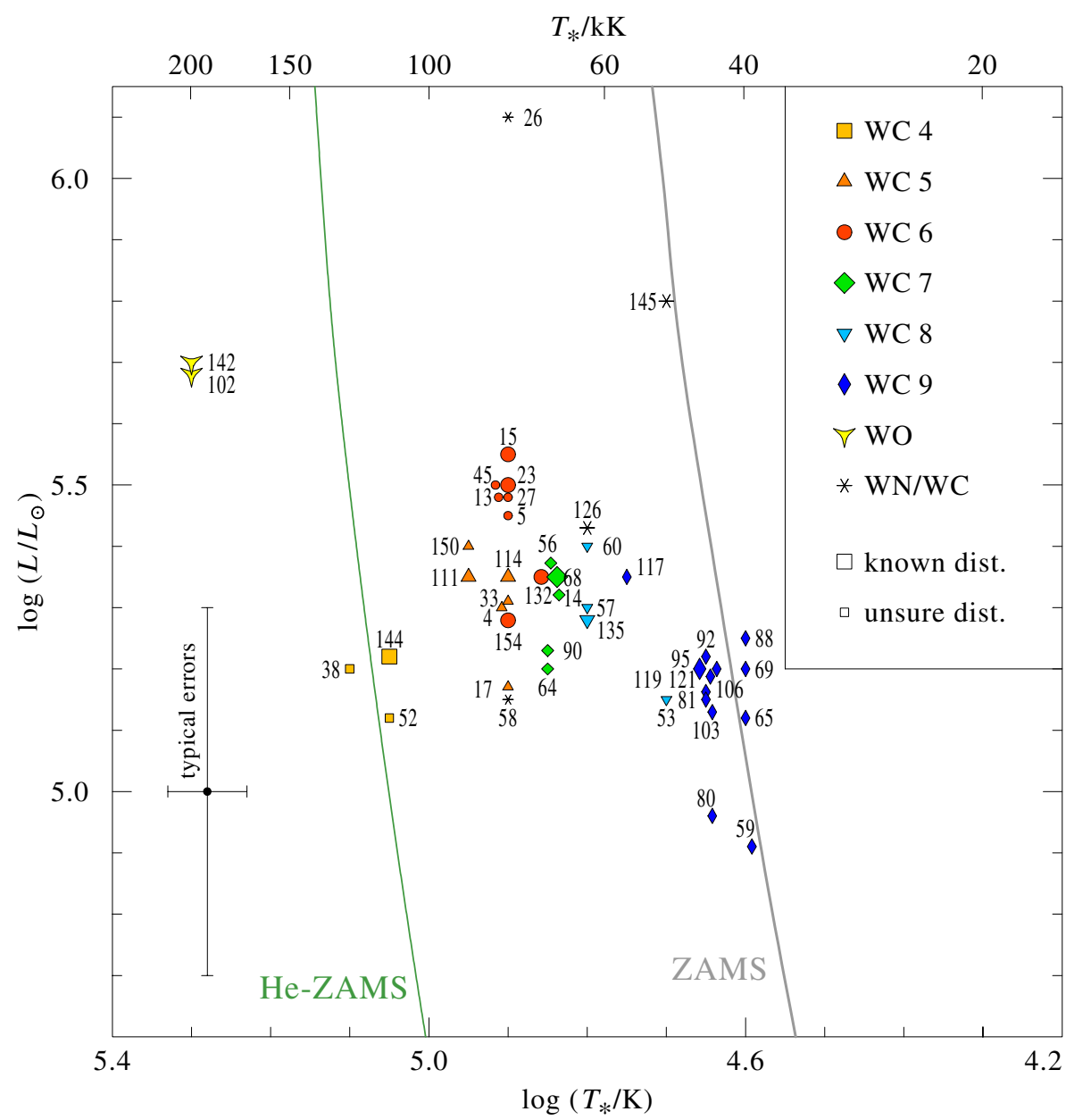

Fig. 7. Hertzsprung-Russell diagram of the Galactic WC stars analyzed in this work. Stars with known distances are represented by larger symbols. The symbols are labeled with the WR catalog number (van der Hucht 2001) of the corresponding stars. grids with fixed $v_{\infty}$. The individual values are inserted, however, when Eq. (2) is applied.

We have so far presented those results derived from fits of the normalized line spectra. We now consider parameters which involve absolute dimensions, namely $R_{*}, \dot{M}$, and $L$, and those required to fit the SED in terms of absolute fluxes.

The color excess $E_{b-v}$, for which we use the monochromatic colors defined by Smith (1968), of the best SED fit is included in Table 4. Further details, i.e. the applied reddening law and its $R_{V}$ parameter if applicable, are printed on the fit plot shown for each star in the appendix.

As explained above in Sect.4, the SED fit relies on either the adopted distance or absolute brightness, as indicated by the direction of the little arrows between the respective columns in Table 4.

The basic free parameter of the SED fit was the stellar luminosity. The models, which were all calculated for a fixed luminosity of $\log L / L_{\odot}=5.3$, were scaled to match the observation. The parameters $L$ and $T_{*}$ were used to derive the stellar radius from Eq. (1), and from the "transformed radius" $R_{\mathrm{t}}$ we obtained the mass-loss rate via Eq. (2) under the assumption of $D=10$. The final values of $R_{*}, \dot{M}$, and $\log L$ are included in Table 4 .

From the luminosities and the stellar temperature, we can construct the empirical Hertzsprung-Russell diagram (HRD, Fig. 7). The stars with distances known from cluster or association memberships are represented by larger symbols, because their luminosities are more trustworthy than those relying on the subtype calibration.

As one can see, the WC stars are located between the hydrogen and helium zero-age main-sequence (ZAMS), except for some of the WC stars that fall on the cooler side of the hydrogen ZAMS. The two WO stars have temperatures above those that would be expected from the He-ZAMS. The WC subtypes form a sequence in the HRD, from the WC9 stars, which are the coolest stars with the lowest luminosities, to the WC4 stars, which are hotter and more luminous. It is unclear whether the scatter is intrinsic to the stars, or caused by wrong distances. Taking the luminosities at face value, they range from about $\log L / L_{\odot}=5.0$ for the faintest WC9 star to about $\log L / L_{\odot}=5.8$ for the brightest WC6 star and the two WOs. Two of the WN/WC transition type stars (WR 26 and WR 145) have exceptionally high luminosities, which are rather typical of WN stars. The HRD positions are discussed further in Sect. 6 as regards stellar evolution.

All our program stars show strong mass-loss. The empirical mass-loss rates from Table 4 are plotted versus their corresponding luminosities in Fig. 8, and follow a tight linear correlation in the double-logarithmic diagram, except for the WO stars. This is unsurprising if we remember that the WC stars basically follow the proportionality $R_{\mathrm{t}} \propto T_{*}^{2}$ (cf. Eq. (5)). If all stars had the same $v_{\infty}$, this would yield the mass loss-luminosity relation $\dot{M} \propto L^{3 / 4}$. This slope with a power of 3/4 was already suggested by Gräfener et al. (1998), who combined analyses from WC stars in the LMC and the Galaxy. The actual trend in Fig. 8 is slightly steeper, because $v_{\infty}$ also increases towards the earlier subtypes. The least square fit to our data gives

$\log \frac{\dot{M}}{M_{\odot} / \mathrm{yr}}=(0.83 \pm 0.11) \log \frac{L}{L_{\odot}}-9.10 \pm 0.57$. 


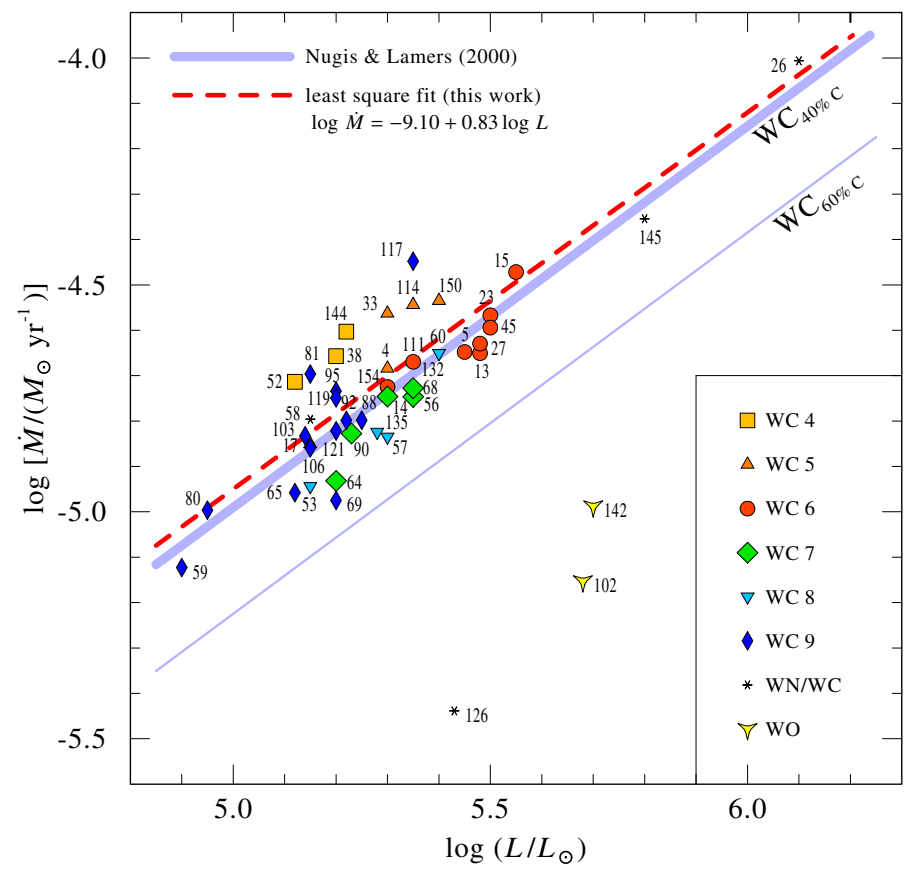

Fig. 8. Empirical mass-loss rates versus luminosity for the analyzed Galactic WC stars. The subtypes are distinguished by different symbols as explained in the inlet. The numbers beside the symbols identify each star by its number in the WR catalog (van der Hucht 2001). The red-dashed red line gives the least square fit to these results. The full, shaded lines represent the relations proposed by Nugis \& Lamers (2000) for WC stars with $40 \%$ and $60 \%$ carbon, respectively.

Our empirical data confirm amazingly well the relation suggested by Nugis \& Lamers (2000) - cf. Eq. (21) therein - based on the empirical data available at that time, when we assume a heavy element mass fraction in their formula of $45 \%$ (thick blue line in Fig. 8). The value of $45 \%$ results from our model assumptions of $40 \%$ carbon and $5 \%$ oxygen.

Two more columns are included in Table 4. The current stellar mass $M$ is deduced from the luminosity. For WC stars, the corresponding relation from Langer (1989) was applied, while for the transition types we adopted the relation for hydrogen-free WN stars from the same reference. The last column in Table 4 lists the values for the wind efficiency parameter $\eta$, defined as the ratio of wind momentum $\dot{M} v_{\infty}$ and photon momentum $L / c$ per time:

$\eta:=\frac{\dot{M} v_{\infty} c}{L}$

The "single scattering limit", at which every photon is scattered exactly once to accelerate the wind, corresponds to $\eta=1$. Hence, each photon has to be scattered on average $\eta$ times to drive the wind with the empirical parameters given in the table. Gräfener \& Hamann (2005) constructed a hydrodynamically self-consistent model for the WC5 star WR 111 that achieved $\eta=$ 2.54 , thus showing that efficiency values of a few can be reached theoretically when multiple-scattering effects are properly taken into account. However, it seems questionable whether values of $\eta \gtrsim 5$ can be explained. In our results many stars have wind efficiencies of $\eta>5$, or even $\eta>10$, especially among the early WC subtypes. Interestingly, the two WO stars have only moderate $\eta$ values.

Possible solutions to this wind-driving problem include: (a) multiple scattering is even more efficient than hitherto thought, (b) there is an additional, yet unidentified wind-driving mechanism, and (c) our mass-loss rates are drastic overestimates because the clumping factor is much higher than the value of $D=10$ that we adopted.

An individual and detailed determination of the chemical composition of our program stars is beyond the scope of the present paper. For the WC stars, we checked that the composition adopted for our model grid (with $40 \%$ carbon, cf. Table 2) is roughly adequate. This can best be seen from the neighboring "diagnostic line pair" He II $5412 \AA$ and C IV $5470 \AA$. These two lines form in the same zone of the wind and display a very similar dependence on $T_{*}$ and $R_{\mathrm{t}}$, while the ratio of their line strengths depends sensitively on the $\mathrm{C} / \mathrm{He}$ abundance ratio. We find that a mass fraction ratio $\mathrm{C}: \mathrm{He}$ of $40: 55$ is adequate for all WC stars in our sample, including the WC9 subtypes. As suggested by Crowther et al. (2006), there is no indication that WC9 stars are chemically less evolved than the earlier subtypes.

Overall, models with an oxygen mass fraction of $5 \%$ seem to be appropriate for WC stars. However, the situation for the individual oxygen-line fit differs. The O Iv-line at $3411 \AA$ is usually nicely reproduced, while the fit quality of the O v line at $5590 \AA$, which is used for classification, varies even within the subtypes. For the WC4 stars WR 52 and WR 144, the line is significantly stronger. Thus, we used a model with an enhanced oxygen fraction of $15 \%$. This raises the question of whether there might be an oxygen trend throughout the WC subtype sequence. There are indeed some stars of the subtypes WC5 and WC6 where we see that O v $5590 \AA$ is significantly stronger than predicted by the model, but there are also several examples where this line is perfectly reproduced with no more than the standard $5 \%$ oxygen mass fraction. We therefore conclude that there is no general increase in oxygen throughout the WC sequence, although there may possibly be an increate for the WC4 subtype.

The WO stars, however, require models with even more oxygen, roughly $30 \%$ (by mass) instead of the 5\% chosen for the WC grid. Interestingly the carbon abundance does not increase but stays at $40 \%$. The remaining approximately $30 \%$ of the mass consists of helium, as the fraction of iron group elements is also the same as in the WC models, namely $0.16 \%$.

The spectra of the three WN/WC transition-type stars in our sample could not be fitted with models of WC-type composition. Their spectral appearance resembles more those of the WN than the WC stars. To simulate these spectra, we started from our models of hydrogen-free WN stars (see Hamann et al. 2006). These models contain mostly $(98 \%)$ helium, plus $1.5 \% \mathrm{~N}$ and only $0.01 \% \mathrm{C}$. While this nitrogen abundance also complies with the $\mathrm{N}$ features in our WN/WC spectra, the carbon abundance has to be increased in order to reproduce the $\mathrm{C}$ lines.

We found that the adequate carbon abundances differ from star to star in this very special group. In particular, WR 58 (WN4/WCE) and WR 145 (WN7/WCE) contain a small amount of carbon, namely $0.1 \%$ and $0.5 \%$ by mass, respectively. In contrast, WR 26 (WN7/WCE) even requires models with a carbon mass fraction as high as $20 \%$ to reproduce its spectrum. We note that we have omitted oxygen in our WN/WC special models to accelerate our calculations, which seems justified because the observed WN/WC spectra show hardly any oxygen features.

The WC/WN star WR 126 is unique to our sample. The only notable emission lines are the C-He-blend around $4650 \AA$, the C Iv $5808 \AA$-line, and three minor lines. Two of them can easily be associated with He II. The peak around $7100 \AA$, however, does not match any line usually seen in WC spectra. However, WN stars display a blend of N Iv lines in this place. For this 
Table 4. Parameters of the Galactic single WC stars.

\begin{tabular}{|c|c|c|c|c|c|c|c|c|c|c|c|c|c|}
\hline WR & Subtype & $\begin{array}{c}T_{*} \\
{[\mathrm{kK}]}\end{array}$ & $\begin{array}{c}\log R_{\mathrm{t}} \\
{\left[R_{\odot}\right]}\end{array}$ & $\begin{array}{c}v_{\infty} \\
{\left[\mathrm{km} \mathrm{s}^{-1}\right]}\end{array}$ & $\begin{array}{c}E_{b-v} \\
{[\mathrm{mag}]}\end{array}$ & $\begin{array}{l}\text { D.M. } \\
\text { [mag] }\end{array}$ & & $\begin{array}{c}M_{v} \\
{[\mathrm{mag}]}\end{array}$ & $\begin{array}{c}R_{*} \\
{\left[R_{\odot}\right]}\end{array}$ & $\begin{array}{c}\log \dot{M} \\
{\left[M_{\odot} / \mathrm{yr}\right]}\end{array}$ & $\begin{array}{c}\log L \\
{\left[L_{\odot}\right]} \\
\end{array}$ & $\begin{array}{c}M^{a} \\
{\left[M_{\odot}\right]}\end{array}$ & $\eta$ \\
\hline 102 & WO2 & 200 & 0.4 & 5000 & 1.08 & 12.39 & $\rightarrow$ & -1.71 & 0.58 & -5.15 & 5.68 & 19 & 3.6 \\
\hline 142 & WO2 & 200 & 0.3 & 5000 & 1.43 & 10.45 & $\rightarrow$ & -2.49 & 0.59 & -4.99 & 5.7 & 20 & 5.0 \\
\hline 38 & WC4 & 126 & 0.1 & 3200 & 1.11 & 14.20 & $\leftarrow$ & -3.34 & 0.84 & -4.66 & 5.2 & 10 & 21.9 \\
\hline 52 & WC4 & 112 & 0.2 & $3225^{b}$ & 0.56 & 11.35 & $\leftarrow$ & -3.34 & 0.96 & -4.71 & 5.12 & 9 & 23.3 \\
\hline 144 & WC4 & 112 & 0.2 & 3500 & 1.6 & 11.3 & $\rightarrow$ & -3.34 & 1.08 & -4.60 & 5.22 & 11 & 25.9 \\
\hline 4 & WC5 & 79 & 0.5 & $2528^{c}$ & 0.6 & 11.83 & $\leftarrow$ & -4.12 & 2.37 & -4.68 & 5.3 & 12 & 12.9 \\
\hline 17 & WC5 & 79 & 0.5 & $2231^{c}$ & 0.31 & 13.11 & $\leftarrow$ & -4.12 & 1.99 & -4.85 & 5.15 & 10 & 11.0 \\
\hline 33 & WC5 & 79 & 0.5 & $3342^{c}$ & 0.6 & 14.07 & $\leftarrow$ & -4.12 & 2.37 & -4.56 & 5.3 & 12 & 22.5 \\
\hline 111 & WC5 & 89 & 0.4 & $2398^{c}$ & 0.34 & 11.0 & $\rightarrow$ & -4.16 & 1.99 & -4.67 & 5.35 & 12 & 11.3 \\
\hline 114 & WC5 & 79 & 0.5 & 3200 & 1.35 & 11.5 & $\rightarrow$ & -4.08 & 2.51 & -4.54 & 5.35 & 12 & 20.1 \\
\hline 150 & WC5 & 89 & 0.4 & 3000 & 0.8 & 13.83 & $\leftarrow$ & -4.12 & 2.11 & -4.53 & 5.4 & 13 & 17.1 \\
\hline 5 & WC6 & 79 & 0.5 & $2120^{c}$ & 0.85 & 12.17 & $\leftarrow$ & -4.42 & 2.81 & -4.65 & 5.45 & 14 & 8.3 \\
\hline 13 & WC6 & 79 & 0.5 & 2000 & 1.21 & 13.24 & $\leftarrow$ & -4.42 & 2.91 & -4.65 & 5.48 & 15 & 7.3 \\
\hline 15 & WC6 & 79 & 0.5 & $2675^{c}$ & 1.23 & 11.28 & $\rightarrow$ & -4.60 & 3.16 & -4.47 & 5.55 & 16 & 12.5 \\
\hline 23 & WC6 & 79 & 0.5 & $2342^{c}$ & 0.55 & 11.8 & $\rightarrow$ & -4.64 & 2.98 & -4.57 & 5.5 & 15 & 9.9 \\
\hline 27 & WC6 & 79 & 0.5 & 2100 & 1.4 & 12.63 & $\leftarrow$ & -4.42 & 2.91 & -4.63 & 5.48 & 15 & 8.0 \\
\hline 45 & WC6 & 79 & 0.5 & 2200 & 1.44 & 13.32 & $\leftarrow$ & -4.42 & 2.98 & -4.59 & 5.5 & 15 & 8.7 \\
\hline 132 & WC6 & 71 & 0.6 & 2400 & 1.15 & 13.16 & $\rightarrow$ & -4.38 & 3.15 & -4.67 & 5.35 & 12 & 11.3 \\
\hline 154 & WC6 & 79 & 0.5 & 2300 & 0.78 & 12.2 & $\rightarrow$ & -4.05 & 2.37 & -4.72 & 5.3 & 12 & 10.7 \\
\hline 14 & WC7 & 71 & 0.6 & $2194^{c}$ & 0.65 & 10.55 & $\leftarrow$ & -4.18 & 2.98 & -4.75 & 5.3 & 12 & 9.7 \\
\hline 56 & WC7 & 71 & 0.6 & $2009^{c}$ & 0.7 & 15.25 & $\leftarrow$ & -4.18 & 3.15 & -4.75 & 5.35 & 12 & 7.9 \\
\hline 64 & WC7 & 71 & 0.6 & 1700 & 1.2 & 14.85 & $\leftarrow$ & -4.18 & 2.65 & -4.93 & 5.2 & 10 & 6.2 \\
\hline 68 & WC7 & 71 & 0.6 & 2100 & 1.4 & 12.57 & $\rightarrow$ & -4.18 & 3.15 & -4.73 & 5.35 & 12 & 8.6 \\
\hline 90 & WC7 & 71 & 0.6 & $2053^{c}$ & 0.4 & 9.50 & $\leftarrow$ & -4.18 & 2.75 & -4.83 & 5.23 & 11 & 8.8 \\
\hline 53 & WC8d & 50 & 0.9 & 1800 & 0.75 & 12.29 & $\leftarrow$ & -4.48 & 5.00 & -4.94 & 5.15 & 10 & 7.1 \\
\hline 57 & WC8 & 63 & 0.7 & $1787^{c}$ & 0.38 & 12.72 & $\leftarrow$ & -4.48 & 3.75 & -4.84 & 5.3 & 12 & 6.4 \\
\hline 60 & WC8 & 63 & 0.7 & 2300 & 1.45 & 11.79 & $\leftarrow$ & -4.48 & 4.21 & -4.65 & 5.4 & 13 & 10.1 \\
\hline 135 & WC8 & 63 & 0.6 & $1343^{c}$ & 0.4 & 11.2 & $\rightarrow$ & -4.48 & 3.66 & -4.82 & 5.28 & 11 & 5.2 \\
\hline 59 & WC9d & 40 & 1.0 & 1300 & 2.0 & 10.73 & $\leftarrow$ & -5.17 & 5.94 & -5.12 & 4.9 & 7 & 6.1 \\
\hline 65 & WC9d & 40 & 1.0 & 1300 & 2.0 & 10.73 & $\leftarrow$ & -5.17 & 7.66 & -4.96 & 5.12 & 9 & 5.3 \\
\hline 69 & WC9d & 40 & 1.0 & $1089^{c}$ & 0.55 & 12.39 & $\leftarrow$ & -5.17 & 8.40 & -4.97 & 5.2 & 10 & 3.6 \\
\hline 80 & WC9d & 45 & 0.9 & 1600 & 1.8 & 12.20 & $\leftarrow$ & -5.17 & 4.99 & -5.00 & 4.95 & 8 & 8.9 \\
\hline 81 & WC9 & 45 & 0.8 & 1600 & 1.5 & 11.40 & $\leftarrow$ & -5.17 & 6.28 & -4.70 & 5.15 & 10 & 11.2 \\
\hline 88 & WC9 & 40 & 1.0 & 1500 & 1.4 & 12.13 & $\leftarrow$ & -5.17 & 8.89 & -4.80 & 5.25 & 11 & 6.6 \\
\hline 92 & WC9 & 45 & 0.8 & $1121^{c}$ & 0.52 & 13.60 & $\leftarrow$ & -5.17 & 6.81 & -4.80 & 5.22 & 11 & 5.3 \\
\hline 95 & WC9d & 45 & 0.9 & 1900 & 1.74 & 11.61 & $\rightarrow$ & -5.17 & 6.66 & -4.73 & 5.2 & 10 & 10.9 \\
\hline 103 & WC9d & 45 & 0.8 & $1190^{b}$ & 0.52 & 11.90 & $\leftarrow$ & -5.17 & 6.21 & -4.83 & 5.14 & 10 & 6.2 \\
\hline 106 & WC9d & 45 & 0.8 & 1100 & 1.2 & 12.43 & $\leftarrow$ & -5.17 & 6.28 & -4.86 & 5.15 & 10 & 5.3 \\
\hline 117 & WC9d & 56 & 0.6 & 2000 & 1.56 & 12.95 & $\leftarrow$ & -5.17 & 5.00 & -4.45 & 5.35 & 12 & 15.7 \\
\hline 119 & WC9d & 45 & 0.8 & 1300 & 0.9 & 13.98 & $\leftarrow$ & -5.17 & 6.66 & -4.75 & 5.2 & 10 & 7.2 \\
\hline 121 & WC9d & 45 & 0.8 & 1100 & 1.40 & 11.84 & $\leftarrow$ & -5.17 & 6.66 & -4.82 & 5.2 & 10 & 5.1 \\
\hline 26 & WN7/WCE & 79 & 0.6 & 2700 & 1.25 & 15.19 & $\leftarrow$ & -5.67 & 5.95 & -4.01 & 6.1 & 37 & 10.4 \\
\hline 58 & WN4/WCE & 79 & 0.5 & 1600 & 0.55 & 14.50 & $\leftarrow$ & -3.84 & 1.99 & -4.80 & 5.15 & 10 & 8.9 \\
\hline 126 & WC5/WN & 63 & 1.2 & 2000 & 0.95 & 13.22 & $\rightarrow$ & -3.82 & 4.35 & -5.44 & 5.43 & 14 & 1.3 \\
\hline 145 & WN7/WCE & 50 & 0.9 & 1440 & 1.86 & 11.3 & $\rightarrow$ & -6.38 & 10.57 & -4.35 & 5.8 & 25 & 5.0 \\
\hline
\end{tabular}

Notes. ${ }^{(a)}$ Masses are calculated from luminosity after Langer (1989) using his WC relation for WC stars and his WNE relation for WN/WC stars. ${ }^{(b)}$ Prinja et al. (1990). ${ }^{(c)}$ Niedzielski \& Skorzynski (2002). The arrows indicate whether we infer the absolute magnitude $\left(M_{v}\right)$ from a known distance modulus D.M., or whether the distance is calculated from $M_{v}$ as obtained by the subtype magnitude calibration (cf. Table 3).

reason, this star is classified as WC5/WN. For our fit, we used a nitrogen-free WC star model with $20 \%$ (i.e. reduced) carbon mass fraction and $5 \%$ oxygen.

Hence, it seems that for the transition-type stars neither the carbon abundance nor the position in the $\log R_{\mathrm{t}}-\log T_{*}$-plane (see Fig. 6) correlates with the spectral subtype.

Summarizing the results for our whole sample, we found that all parameters show a close correlation with the spectral subtype (except for the transition types to WN). In Table 6, we compile the mean parameters for each subtype. These results are discussed further in Sect. 6 as regards the stellar evolution.

\subsection{Error estimations}

As explained in Sect. 5.1, the spectroscopic parameters $\left(T_{*}\right.$, $R_{\mathrm{t}}$ ) were determined by selecting the best-fitting synthetic line 
spectrum from our model grids. Those grids are spaced by 0.05 in $\log T_{*}$ and 0.1 dex in $R_{\mathrm{t}}$, which corresponds to our experience that this is roughly the accuracy with which the optimum line fit can be identified.

For very dense winds $\left(\log R_{\mathrm{t}} / R_{\odot} \lesssim 0.4\right)$, all emergent radiation originates from the fast-moving parts of the stellar wind, as discussed in Sect. 5.1. Hence, in this regime of parameter degeneracy only the product $R_{\mathrm{t}} T_{*}^{2}$ is constrained by the line fit.

While the spectroscopic parameters are derived from the (normalized) line spectrum, the luminosity is obtained by fitting the SED. For most of our program stars, the photometrically calibrated observations cover a wide spectral range from the UV to the IR, hence the color excess $E_{b-v}$ can be determined to an accuracy of $\pm 0.02 \mathrm{mag}$. The uncertainty in the distance is larger, which enters the derived luminosity $\propto d^{2}$ and the mass-loss rate $\propto d^{3 / 2}$. For many of our stars we must rely on the assumption that the $v$ magnitude is constant per subtype (Sect. 4). This whole calibration, in turn, is based on those stars that presumably belong to a certain open cluster or association. Apart from the possibility that the membership assignment may be erroneous in individual cases, the distance to the clusters or associations are also continue to be debated between different authors, as particularly mentioned in Appendix A. To our impression, the distance moduli remain uncertain by about $0.75 \mathrm{mag}$, leading to an error margin of \pm 0.3 dex for the luminosity.

Systematic errors of our analyses are extremely difficult to quantify. Atomic data are one source of uncertainties. A more important question is the degree to the basic model assumptions, such as spherical symmetry and homogeneity, are adequate for real stars. A plethora of recent work deals with clumping in hot star winds (cf. Hamann et al. 2008). Clumping can bias in particular the empirical mass-loss rates.

\subsection{Comparison with previous WC analyses}

A larger sample of Galactic WC stars were analyzed in our group by Koesterke \& Hamann (1995), yet with un-blanketed PoWR model atmospheres. Their results did not reveal the clear correlation between the subtypes and the stellar parameters that we see now. The former analysis was partly confused by a couple of objects that have since been identified as binaries. In the case of WN stars, the improvement from un-blanketed to iron-lineblanketed models led to about $10 \mathrm{kK}$ higher stellar temperatures (Hamann et al. 2006). Surprisingly, this is not the case for the WC stars. The newly obtained mass-loss rates are lower, mainly because of the microclumping correction (cf. Sect. 3).

A couple of Galactic WC stars were analyzed more recently with the help of the line-blanketed CMFGEN model atmosphere code by Hillier \& Miller (1998). WR 111 (WC5) was analyzed by Hillier \& Miller (1999). Dessart et al. (2000) studied another four Galactic WC stars, two of them, WR 90 (WC7) and WR 135 (WC8), being single stars. Crowther et al. (2006) analyzed the WC9d star WR 103. Their results are compared in Table 5 with those obtained in this work ("t.w."). The agreement among the stellar temperatures is very good. We tend to obtain slightly higher luminosities by 0.1 dex. The mass-loss rates have a considerable scatter; we note that they were derived with the same assumption for the clumping parameter $(D=10)$ as in this work.

Interestingly, Dessart et al. (2000) also concluded that there is no correlation between stellar temperature and subtype, as in our Koesterke \& Hamann (1995) paper, and in sharp contrast to our present result. The reason is obviously that their study was also confused by binaries that have diluted emission lines.

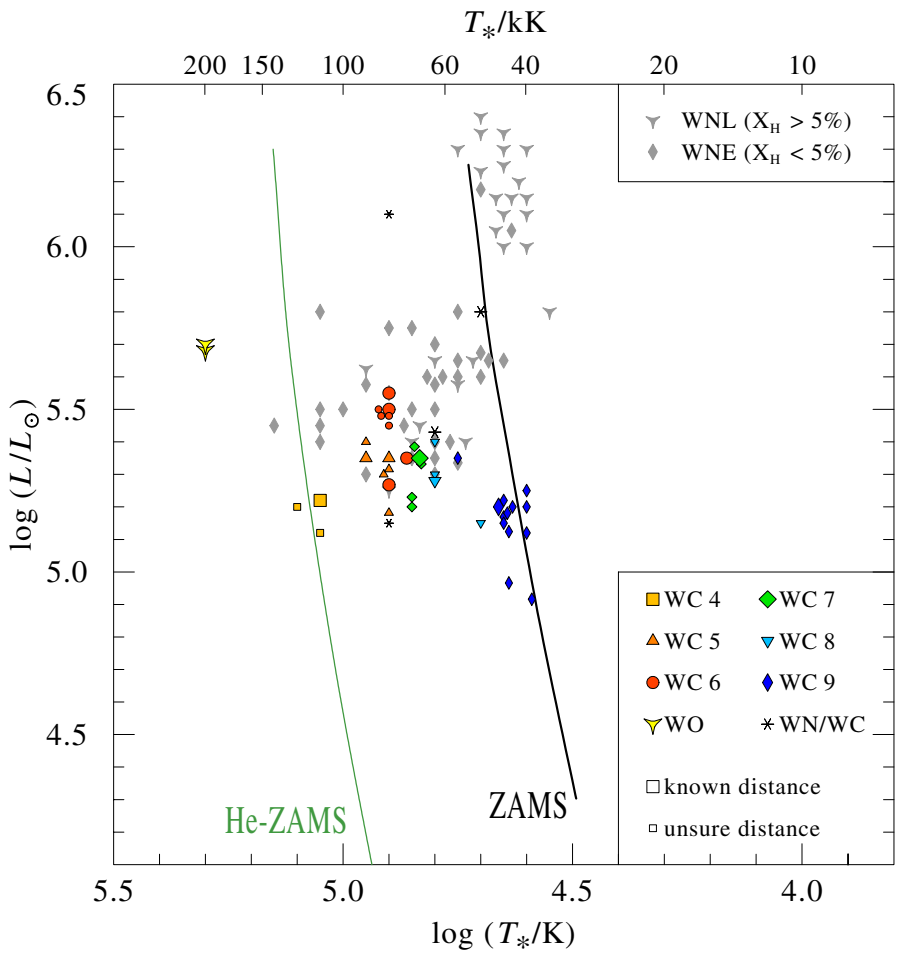

Fig. 9. HRD with the WC star positions from this work and the Galactic WN star positions from Hamann et al. (2006).

Table 5. Comparison of stellar parameters with previous analyses

\begin{tabular}{ccccccc}
\hline \hline WR & Subtype & Ref. & $\begin{array}{c}T_{*} \\
{[\mathrm{kK}]}\end{array}$ & $\begin{array}{c}\log L \\
{\left[L_{\odot}\right]}\end{array}$ & $\begin{array}{c}v_{\infty} \\
{\left[\mathrm{km} \mathrm{s}^{-1}\right]}\end{array}$ & $\begin{array}{c}\log \dot{M} \\
{\left[M_{\odot} / \mathrm{yr}\right]}\end{array}$ \\
\hline 111 & WC5 & t.w. & 89 & 5.35 & $2398^{4}$ & -4.67 \\
& & 1 & 91 & 5.3 & 2300 & -4.8 \\
90 & \multirow{2}{*}{ WC7 } & t.w. & 71 & 5.23 & $2053^{4}$ & -4.83 \\
& & 2 & 71 & 5.5 & 2045 & -4.6 \\
135 & \multirow{2}{*}{ WC8 } & t.w. & 63 & 5.28 & $1343^{4}$ & -4.82 \\
& & 2 & 63 & 5.2 & 1400 & -4.9 \\
103 & \multirow{2}{*}{ WC9d } & t.w. & 45 & 5.14 & $1190^{5}$ & -4.83 \\
& & 3 & 48 & 4.90 & 1140 & -4.50 \\
\hline
\end{tabular}

References. (1) Hillier \& Miller (1999); (2) Dessart et al. (2000); (3) Crowther et al. (2006); ${ }^{(4)}$ Niedzielski \& Skorzynski (2002); ${ }^{(5)}$ Prinja et al. (1990).

\section{Stellar evolution}

To discuss the evolutionary origin of the WC stars, we now compare their empirical positions in the HRD with those of their potential progenitors, the WN stars. The results of this work are shown in Fig. 9, together with those of the Galactic WN stars studied by Hamann et al. (2006). The WN stars form two distinct groups, the very luminous "late" subtypes (WNL) with a significant fraction of hydrogen in their atmosphere, and the less-luminous "early" subtypes (WNE), most of which are completely hydrogen-free.

Like the WNE stars, the WC stars group at the left, hotter side of the ZAMS, while the hydrogen-containing WNL stars lie on the cooler side of the main sequence. The WO stars are even hotter than stars on the helium zero-age main sequence would be. We note that the luminosity range for the WC stars of early subtypes (WC4-7) and two WO stars is almost the same as for the hydrogen-free WNE subclass. The latest WC subtypes, WC8-9, are less luminous than any WN star. The large group of 
WNL stars, which are very luminous $\left(\log L / L_{\odot} \geq 6\right)$, have no counterparts to similarly luminous WC stars.

These results provide an interesting test case for stellar evolution models. The stellar evolution tracks calculated by Meynet \& Maeder (2003) from the Geneva group are the standard reference for massive-star evolution. In Figs. 10 and 11, we compare the HRD of our WC sample with the tracks of Meynet \& Maeder (2003) in the different versions with and without accounting for rotation, respectively. According to the chemical composition at the surface, the tracks are drawn in different styles: hydrogenrich phases are represented by thin black lines. When the hydrogen mass fraction of the atmosphere drops below $40 \%$ as in the WNL stars, the track is shown as a thick gray line. The hydrogen-free WN stage $\left(X_{\mathrm{H}}<5 \%\right)$ is indicated by a thick dark line. In the WC stage (thick black/blue line), carbon reaches more than $20 \%$ in the stellar envelope. We chose this limit to be higher than in Hamann et al. (2006) to ensure that the WC part of the tracks exclude any WN/WC transition stage.

The evolutionary tracks predict that in the non-rotating scenario, massive stars with an initial mass of $60 M_{\odot}$ and above undergo a luminous blue variable (LBV) phase before entering the WNL stage. However, in the scenarios with rotation these stars skip the LBV phase and enter the WNL stage directly after the main sequence (Meynet \& Maeder 2003). Afterwards, the WNL stars evolve to hydrogen-free WNE stars at slightly lower luminosities, depending on the initial mass. They eventually enter the WC stage at roughly the helium main-sequence. According to the continuous mass-loss and the mass-luminosity relation, their luminosity decreases by a few tenths in $\log L$ before the gravitational core-collapse.

Comparing now the Geneva tracks with our empirical HRD (Figs. 10 and 11), we find relatively good agreement for the two WO stars of our sample. In particular the tracks without rotation and for initial masses $\geq 60 M_{\odot}$ reach very hot and luminous endpoints prior to the supernova (SN) explosion.

For the WC stars, however, the comparison ends less favorably. The first problem is posed by the luminosities. The evolutionary calculations without rotation (Fig. 11) predict that only tracks for initial masses above $37 M_{\odot}$ return from the red supergiant (RSG) stage to the blue side of the HRD. The implied luminosities are higher than those inferred from our WC sample. The tracks with rotation (Fig. 10) predict WR stars for initial masses above $22 M_{\odot}$. Hence, the tracks are at least compatible with the most luminous WC stars of our sample, the WC6 subtypes. The bulk of WC stars, however, are less luminous than predicted by any of the tracks in their post-RSG phase. If we apply these relations between mass and luminosity from the Geneva tracks, the conclusion is that WC stars mainly arise from initial masses lower than $40 M_{\odot}$.

Our comparison of effective temperatures has some caveats, since these depend on the choice of the reference radius. The evolutionary tracks are plotted over the stellar effective temperature that refers to the hydrostatic stellar core. Our stellar temperature $T_{*}$ refers to the radius of Rosseland optical depth 20 (cf. Sect. 3). For the adopted velocity (and implied density) structure, this is close to the hydrostatic radius for most of the bestfit models. However, there are indications (Ishii et al. 1999; Petrovic et al. 2006) that WR stars may have very extended, sub-photospheric layers, i.e. the hydrostatic core may actually be much smaller and hotter than derived from the observation of the stellar atmosphere. Moreover, for stars in the domain of the parameter degeneracy (i.e. WC4-5) discussed in Sect. 5, the hydrostatic stellar radius can only be estimated from a significant inward extrapolation of the velocity field into optically thick

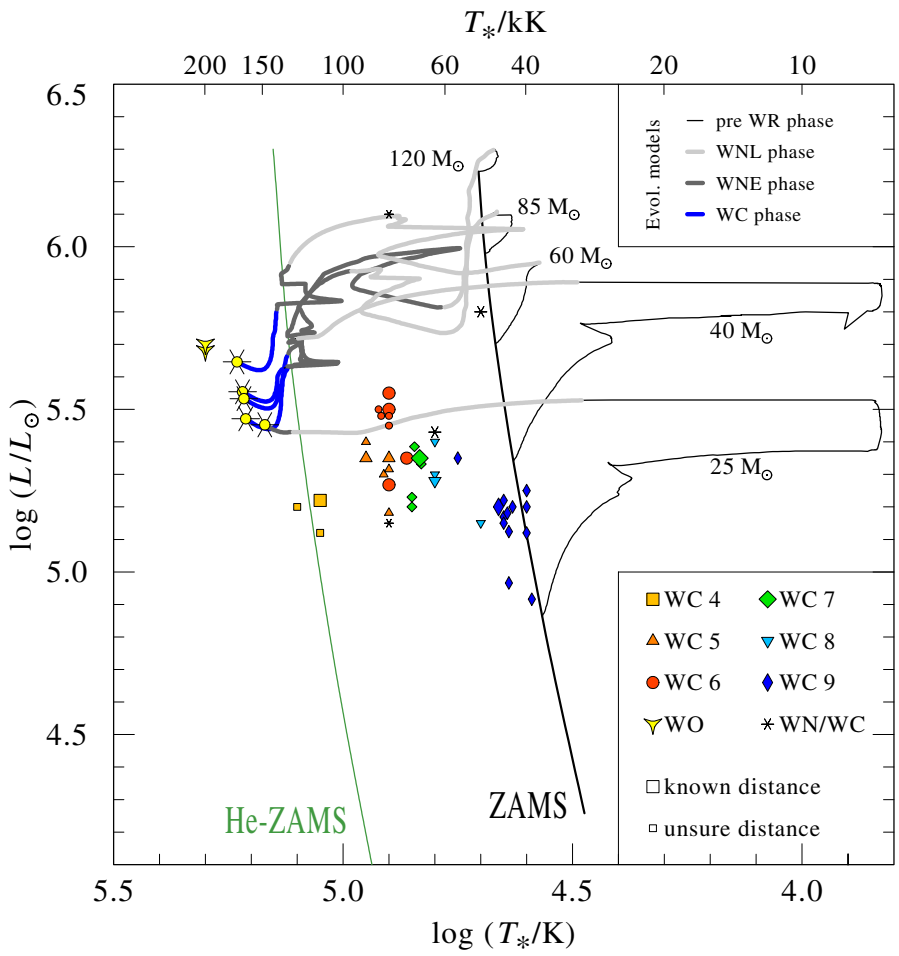

Fig. 10. HRD with the WC star positions and the tracks from Meynet \& Maeder (2003) with an initial rotational velocity of $v_{\text {rot }}=300 \mathrm{~km} \mathrm{~s}^{-1}$. The thick lines indicate the WR phases of the tracks.

layers, which can lead to either an over- or under-estimation of the corresponding effective temperature.

The empirical HRD of the WC stars shows a temperature gap between the helium main-sequence and the WC positions. While the WO subtypes are located to the left of the He-ZAMS (presumably indicating the position of a hypothetical CO-ZAMS), the later WC subtypes are progressively cooler. The later the WC subtype, the lower the luminosity (implying a lower current mass according to the mass-luminosity relation), and the larger must be the sub-photospheric layer enhancing the stellar radius on top of the hydrostatic core.

One could argue that all luminosity values are affected by (almost) all distance values for Galactic WR stars being inferred from cluster memberships. Even if some of the distances for individual stars were wrong due to a false assignment to an association or cluster, only their HRD positions would change while the overall picture would not be drastically affected, as long as cluster distances are not systematically underestimated.

In contrast to the Geneva stellar evolution models, Vanbeveren et al. (1998) showed that it is indeed possible to reach the WR stage for stars with a minimum initial mass of only $15 M_{\odot}$, if the mass-loss rate during the red super-giant (RSG) stage is higher than given by the formula of de Jager et al. (1988) adopted for the Geneva tracks. When adopting a higher RSG mass-loss, the resulting tracks (see Fig. 12) predict, for instance, a luminosity of $\log L / L_{\odot} \approx 5.2$ for a star with $M_{\text {init }}=20 M_{\odot}$ entering the post-RSG stage, and a final luminosity of $\log L / L_{\odot} \approx 4.8$ before explosion. Although in the models of Vanbeveren et al. (1998), WC phases only occur for stars with $M_{\text {init }} \geq 25 M_{\odot}$, the tracks cover almost the whole luminosity range of our WC sample. As for the results from the Geneva tracks, there is a discrepancy in the temperature $T_{*}$ between the tracks and the evolutionary models. To date, none of the evolutionary calculations performed have been able 


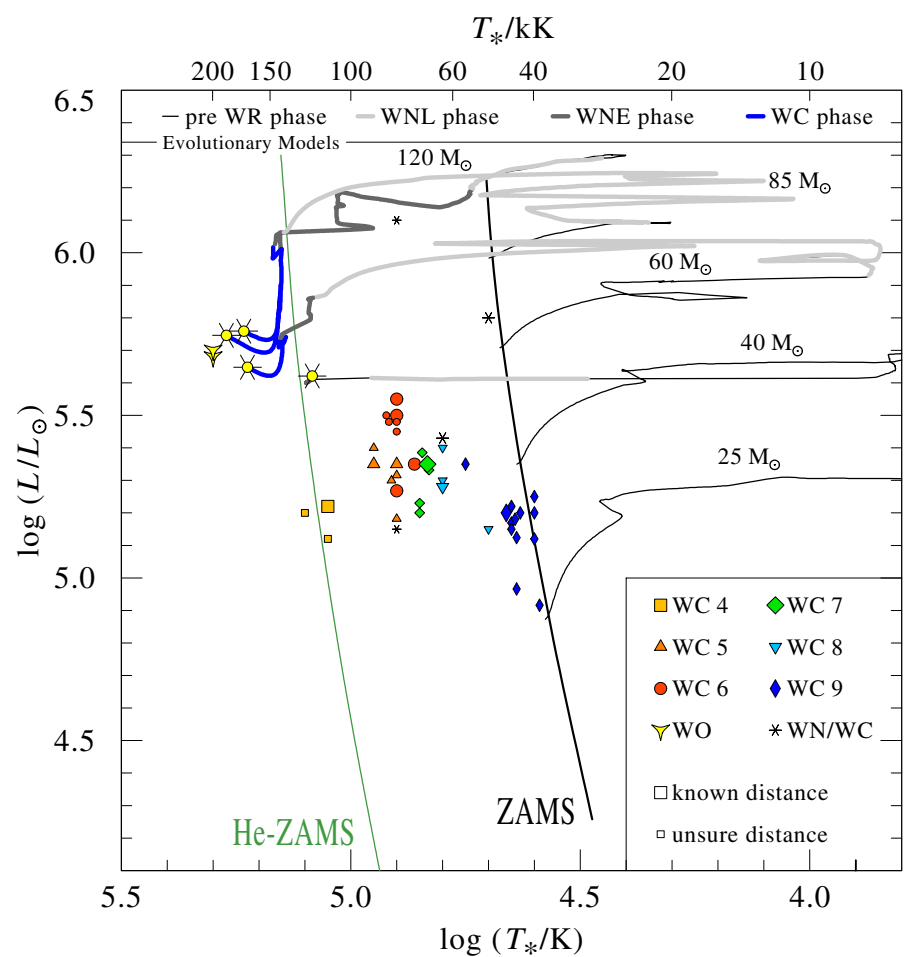

Fig. 11. HRD with the WC star positions and the tracks from Meynet \& Maeder (2003) without rotation. The thick lines indicate the WR phases of the tracks.

to fully explain the obtained HRD positions, as they fail to reproduce the temperatures but provide enough indications that WC stars originate from a lower mass range. Both the Geneva and the Vanbeveren models imply that the WC phase is close to the He-ZAMS. Gräfener et al. (2012) showed that extended sub-photospheric layers are not limited to hydrogen-rich stars on the upper part of the main sequence, but also occur for $\mathrm{H}$ free stars on the He-ZAMS. This happens even in a lower mass regime if the clumping factor is sufficiently high. As briefly discussed above, such an envelope inflation would strongly reduce the effective temperatures of the stars, placing them exactly in the temperature regime we obtained in our work. Inflation might be a key factor in solving the temperature discrepancy.

Another crucial aspect is the highly uncertain mass-loss rates during the RSG stage pointed out by Meynet et al. (2011), which is partly caused by dust. For objects covered with dust, van Loon et al. (2005) discovered mass-loss rates that are significantly higher than those of visually bright ones. Such high rates would lead to stars evolving back from the red supergiant stage to the blue side of the HRD instead of exploding as a type II-P supernova. This would be in line with the observational constraints for type II-P supernovae provided by Smartt et al. (2009), who found several SN II-P progenitors to be red supergiants, but none to have an initial mass of $18 M_{\odot}$ or higher. However, as there are known RSGs at least in the mass range between $18 M_{\odot}$ and $25 M_{\odot}$, it is likely that they do not explode, but instead evolve to blue supergiants and eventually WR stars. Blue supergiants that have previously undergone an RSG stage would be He-enriched. Objects with such an appearance have indeed been found by Przybilla et al. (2010).

Vanbeveren et al. (2007) also compared the parameters of the Galactic WN stars from our previous study (Hamann et al. 2006) with his tracks, which incorporate a higher RSG mass-loss rate (but no rotationally induced mixing), and concluded that their

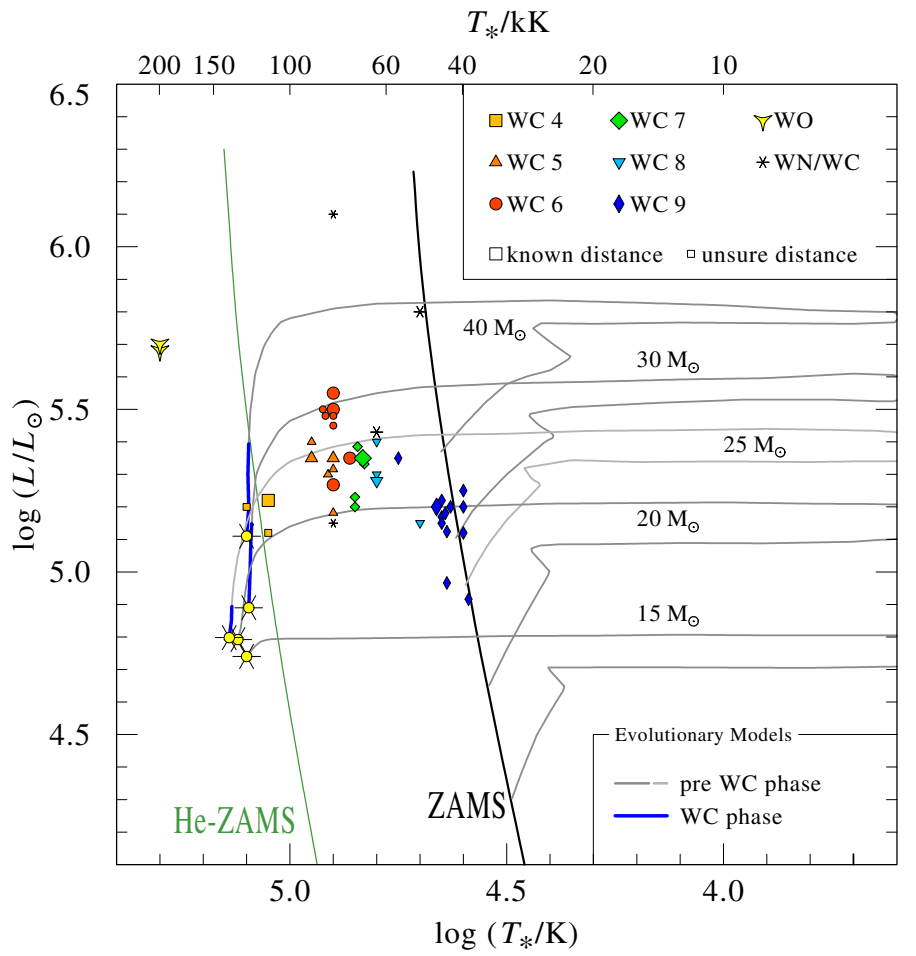

Fig. 12. HRD with the WC star positions and the tracks (thick lines) from Vanbeveren et al. (1998) using higher mass-loss rates during the RSG phase. The track for $M_{\text {init }}=25 M_{\odot}$ is shown in light grey for clarity. In the WC phase, all tracks are drawn as thick dark/blue lines.

own tracks are in much better agreement with our results than the Geneva tracks.

Tracks that lead to WC stars with lower initial masses question the evolutionary scenarios of Conti (1979) and also the revised version of Langer et al. (1994) that WNL stars evolve to WNE stars. The clear separation of WNL and WNE stars in the HRD found by Hamann et al. (2006) suggests instead that WNL stars may not manage to get rid of their hydrogen layers, but explode as type II supernovae either directly after their WNL phase or, more likely, after excursions into the LBV domain. More recent results (e.g. Liermann et al. 2010; Crowther \& Walborn 2011) indeed often discuss or assume that hydrogenrich WNL (also labeled WNh) stars are core-hydrogen burning and cover the high-mass end of the main sequence. Simply put, with increasing initial mass, the spectral types at the high-mass end of the main sequence change from Of to Of/WN and finally WNL.

Moreover, our conclusions as regards stellar evolution can be explained in consistence with the interpretation of supernovae statistics by Smith et al. (2011). They analyzed the different fractions of core-collapse supernovae (CCSN) using the data from the Lick Observatory Supernovae Search (LOSS), covering mostly host galaxies with metallicities similar to Galactic metallicities $\left(0.5-2 Z_{\odot}\right)$. If the WNL stars do explode as supernovae without losing their hydrogen envelope, they will produce a type II supernova instead of the Ib and Ic supernovae that are usually considered to be the end of WR evolution, because there is no hydrogen at all left in the WC stars and little in the WNE stars. Smith (2008) discussed how type IIn supernovae require stars with $M_{\text {init }}>50 M_{\odot}$ and these high mass stars can indeed explode without losing their hydrogen envelope. As type IIn supernovae require huge eruptive mass-loss that exceeds even the high WR mass-loss rates, the primary candidates are 
LBVs, as suggested by Gal-Yam et al. (2007) and eventually proven for SN 2005gl (Gal-Yam \& Leonard 2009). The HRD positions obtained for the Galactic WNL stars (Hamann et al. 2006) corroborate our findings that the hydrogen-rich WNL stars are LBV progenitors which has also been suggested by Smith \& Conti (2008). All of the analyzed Galactic WNL stars seem to have initial masses higher than $60 M_{\odot}$. Their evolutionary sequence in the high mass range should therefore be

Of $\rightarrow$ Of $/ \mathrm{WNL} \leftrightarrow \mathrm{LBV}\left[\rightarrow \mathrm{WNL}_{\mathrm{H}-\text { poor }}\right] \rightarrow \mathrm{SN}$ IIn.

There is an ongoing controversial debate about whether LBVs can be direct SN progenitors. In general, evolutionary calculations do not predict such a direct connection. The LBVs are characterized by their variability, which one cannot directly measure after the star has exploded, as e.g. in the case of SN2005gl (GalYam \& Leonard 2009). Kotak \& Vink (2006) suggested LBVs as progenitors of certain supernovae due to signatures of progenitor variations in the SN spectrum. For SN 2005gj, Trundle et al. (2008) argued that line profiles similar to the observed ones could only be found in LBVs. Finally, luminosity and color, if these data exist, may indicate whether a SN progenitor was a blue star beyond the Humphrey-Davidson limit.

Since we know WNL stars with very different hydrogen abundances (Hamann et al. 2006; Martins et al. 2008; Liermann et al. 2010), it is likely that there are multiple excursions between a quiet WNL stage and LBV eruptions, as indicated by the double-side arrow in Eq. (8).

Dwarkadas (2011) questioned the scenario of LBVs being direct SN progenitors. He argued that the deduced mass-loss rates are usually based on the assumption of $r^{-2}$-density stratifications, which might not be correct. He further argues that there should be at least a short WR phase between an LBV outburst and a SN explosion. For a type IIn supernova, this phase would correspond to a WNL type with a low hydrogen abundance. We therefore add the suffix "H-poor" to this possible post-LBV WNL stage in Eq. (8).

Smith et al. (2011) discussed that there seem to be two ways for LBVs, either by exploding or turning into another WR stage to eventually explode as SN Ib or Ic. Since the results of our WC analyses suggest that WNE and WC stars have progenitors of lower mass, this decision conflict would be solved. For an initial mass range from approximately $20 M_{\odot}$ to $50 M_{\odot}$, the WR stars would arise as post-RSG stars with most of their hydrogen being lost during the RSG stage, possibly skipping a hydrogen-rich WN stage that is not observed for this mass range. The evolutionary sequence leading to WC stars might therefore be

$\mathrm{O} \rightarrow \mathrm{RSG} \rightarrow \mathrm{WNE} \rightarrow \mathrm{WC} \rightarrow \mathrm{SN} \mathrm{Ib} / \mathrm{c}$.

However, there is an upper mass limit for red supergiants. Although the exact value is unclear, it might be somewhere between $25 M_{\odot}$ and $50 M_{\odot}$ (e.g. Meynet \& Maeder 2003; Smartt 2009), meaning that stars with higher initial masses do not become RSGs. Stars in the range above the RSG mass limit but below the WNL mass range might therefore undergo an LBV phase, but do not appear as WNL stars at any earlier stage. In addition, these "lower mass LBVs" do not seem to explode as type II supernovae but instead lose their hydrogen layers and eventually become hydrogen-poor stars, such as WNE, WC, and WO stars. Assuming a mass limit for red supergiants significantly below $50 M_{\odot}$, the evolutionary sequence for the WC stars above would be

$\mathrm{O} \rightarrow \mathrm{LBV} \rightarrow \mathrm{WNE} \rightarrow \mathrm{WC} \rightarrow \mathrm{SN} \mathrm{Ib} / \mathrm{c}$.
Our empirical HRD positions for the Galactic WC stars instead support the post-RSG origin of Eq. (9). All WC stars are located below the $40 M_{\odot}$ tracks, independently of their detailed scenario assumptions. Even if the RSG upper mass limit is somewhat lower, most if not all WC stars of our sample must be attributed to the post-RSG evolution.

Regardless of how the WC stars are produced, if they explode as a SN, it clearly has to be of type Ib or Ic, as these stars are hydrogen-free. While there are known progenitor stars for type II supernovae, there are none for type Ib and Ic. Therefore it is not yet clear if WNE or WC stars will undergo a supernova explosion at all. Instead they might just collapse to a black hole without a high luminosity outburst.

To support the mass ranges for this new sequences, we take a closer look at the discussions from Smith et al. (2011), assuming that WC stars always end up in a supernova. They tried to assign the different core collapse supernova types to mass ranges, so that the fractions fit with a Salpeter IMF. With the assumption all supernovae arise from single-star evolution they end up with a maximum mass of $22 M_{\odot}$ for type II supernovae, even including the SNe IIn here. That would imply that all stars above $22 M_{\odot}$ would completely lose their hydrogen, probably becoming a WNE and finally a WC or WO star. Although this range would fit with the lower end of our WC initial mass range, this scenario ignores the previously suggested WNL evolution and contradicts not only the idea, but also the observation of a $\mathrm{SNe}$ IIn with a high-mass progenitor.

Even though they are only referring to core-collapse supernovae, it is clear that binaries have to be taken into account. In contrast to the assumption that only single-star evolution dominates the $\mathrm{SNe}$ statistic, one could assume that binary evolution is the only possible way for type Ib/c SNe. That would of course assume that all WNE, WC, and WO stars are binary systems or have at least been formed by close binary evolution. Although a number of systems might have formed in this way, it is highly unlikely that all WR stars (except WNL stars) share this origin. Stellar evolution models might not cover the right mass range for these stars yet, but they do show that WNE and WC stars can be formed without requiring close binary evolution.

Smith et al. (2011) therefore presented two ad hoc scenarios combining the ideas of single star and binary evolution. A fraction of $8.8 \%$ of their SN sample are of type IIn while a total fraction of $26 \%$ are type I CCSNe (hereafter labeled SNe Ib/c). Among these $26 \%$, some originate from single stars and others from binaries. As an ad hoc approach, they assumed that the single star fraction should be as large as the SNe IIn fraction, namely $8.8 \%$. The remaining fraction is considered as a result of close binary evolution, together with the SNe IIb, which are actually type Ib SNe with small amounts of hydrogen visible only in the early stage of the SN. For the type II-P and II-L SNe associated with the low initial masses, they ended up with an upper limit to $M_{\text {init }}$ of $23.1 M_{\odot}$. Above this initial mass, all single stars explode as either type Ib/c or IIn supernovae. Were a strict mass limit assumed to separate the $\mathrm{SNe} \mathrm{Ib/c} \mathrm{and} \mathrm{IIn} \mathrm{sce-}$ narios, this would be $M_{\text {init }}=37 M_{\odot}$ under the assumptions stated above. Smith et al. (2011) assigned the SNe IIn to the lower mass range between $23 M_{\odot}$ and $37 M_{\odot}$, but according to the results of this work and the observation of Gal-Yam \& Leonard (2009), it seems likely that the converse is true. The IIn might then belong to stars with $M_{\text {init }}>37 M_{\odot}$, possibly following the evolutionary scenario (8), and the $\mathrm{SNe} \mathrm{Ib} / \mathrm{c}$ come from WC stars with initial masses between 23 and $37 M_{\odot}$, which is not so far from our proposal based on the HRD positions relative to the evolutionary tracks. 
The limits of the mass ranges are of course only rough estimations. Neither our track comparisons, nor the ad hoc assumption of the single star fraction in the $\mathrm{SNe} \mathrm{Ib} / \mathrm{c}$ fraction are based on detailed calculations. The limits therefore can change in both directions, e.g. depending on the values of the RSG mass-loss rates and the number of binaries causing type $\mathrm{Ib} / \mathrm{c} \mathrm{SNe}$. A larger single-star fraction in the ad hoc assumption would decrease the minimum initial mass, while a lower RSG mass loss would raise it. As an additional channel leading to $\mathrm{SNe}$ of type $\mathrm{Ib} / \mathrm{c}$, Smith et al. (2011) suggested underluminous H-poor stars. We consider this idea as speculative, because these objects have never been observed. Admittedly, if they formed by Roche-lobe overflow in close-binary systems, such stars might escape detection. However, on the basis of the SN statistics, the latter case is covered by the binary fraction that was already subtracted from the number of $\mathrm{Ib} / \mathrm{c}$ supernovae. Despite all the remaining uncertainties, the scenario demonstrates that the observed CCSN statistics in general can be explained in-line with our empirical results.

We have so far neglected two stars that do not seem to follow one of the newly introduced scenarios (8) or (9), namely the two WO2 stars WR 102 and WR 142. In contrast to the early WC subtypes they appear as hot, but also very luminous stars. Their positions are close to the end of the tracks of Meynet \& Maeder (2003) with favoring the non-rotational models. Their positions therefore indicate that these two WO stars at least are stars with $M_{\text {init }}>40 M_{\odot}$. The end of each Geneva track is marked by a SN symbol, which is not entirely appropriate as the track calculations end when there is no more helium left in the stellar core. It has been suggested that WO stars might already be in a state of carbon burning (e.g. Barlow \& Hummer 1982). The low number of known WO stars - just four of them in the Milky Way - and the high temperature of the corresponding PoWR model compared to the Geneva track endings support this idea. However, owing to the absence of unambiguously identified helium lines in the optical spectrum, our models might overestimate the helium abundance in the WO stars. Only if these stars were completely helium-free, we would have a clear evidence of a carbon-burning star.

With the sequences (8) and (9), we have evolutionary scenarios for the WC stars with masses of up to $40 M_{\odot}$ or maybe $50 M_{\odot}$ and another for the WNL stars with $M_{\text {init }}>60 M_{\odot}$, suggested by the WNL positions and the SN IIn constraints. The observed WC luminosities also roughly agree with the tracks of Vanbeveren et al. (1998) for this mass range. The tracks do not reproduce the obtained temperatures but this might be due to envelope inflation, which has never been included in any evolutionary track to date. The WO stars might actually originate from stars with initial masses between the two mass bins. To end up as WO star, a star might fellow a scenario close to the one of Langer et al. (1994), which is also a kind of mixture of scenarios (9) and (8)

$$
\mathrm{O} \rightarrow \mathrm{WNL} \rightarrow \mathrm{LBV} \rightarrow \mathrm{WNE} ? \rightarrow \mathrm{WO} \rightarrow \mathrm{SN} \mathrm{Ib} / \mathrm{c} .
$$

In contrast to the original assumption of Langer et al. (1994), the WC phase is skipped here. If the WO stars were to evolve from WC stars, we would expect to see WC stars with even higher luminosities than the WO stars, which are not observed. The situation is not much better for WNE stars, hence this stage is tagged with a question mark in (11) as there are no stars observed in a luminosity range that would correspond to the luminosities of the WO stars. However, this might be just a consequence of the low number statistics, as the WNE lifetimes are a factor of between three and ten shorter than those of the WC stars, according to the calculations of Meynet \& Maeder (2003). In the case of
He-burning WO stars, their lifetimes would be comparable to those of WC stars and with only two known WO2 stars in the Milky Way, the absence of such WNE stars in the corresponding luminosity range would be merely a statistical effect.

The final SN explosion, if there is one, should be of either type Ib or Ic. The latter would assume that such stars have lost all their helium. This is not the case in our current WO models, which contain a helium mass fraction of $30 \%$. However, as mentioned above, we cannot rule out that these stars might already be helium-depleted.

Alternatively, one could imagine that WO stars form from rapidly rotating WNL stars, with a very short or even without a WNE phase. This would be in line with our findings that both WO stars in our sample require a very high rotational velocity of $1000 \mathrm{~km} \mathrm{~s}^{-1}$ to reproduce their round-shaped spectral lines. On the basis of these assumptions, the evolutionary scenario would be

$$
\mathrm{O} \rightarrow \mathrm{WNL} \rightarrow \mathrm{WO} \rightarrow \mathrm{GRB}
$$

Stellar evolution calculations currently support neither scenario (11) nor (12). The most recent Geneva models (Maeder $\&$ Meynet 2011) predict a spin-down during the main-sequence stage, leading to lower rotational velocities at higher masses. However, we do see extremely broad lines in the WO spectra that can only explained by very rapid rotation. We speculate that these stars were either spun up by mass transfer from a companion, or that the transfer and loss of angular momentum remains inadequately described by the current evolutionary models.

In total, the modified scenario of Smith et al. (2011) corroborates our conclusion that WC stars arise from those of lower initial masses than previously expected, as raised by the HRD positions. It is also in line with the assumption that the very luminous WNL stars are core hydrogen-burning stars that will never reach the WNE and WC stages. However, the SN scenario does not include metallicity effects and their binary assumptions are just ad-hoc. As the formation of WR stars might strongly depend on the metallicity of the host galaxy, the SN statistics show a plausible and interesting scenario that is in line with our results, but one that should not be taken as an independent argument for the WC mass range.

Metallicity effects may be illustrated by comparing WC stars in the Galaxy and the Large Magellanic Cloud (LMC). The six WC4 stars in the LMC were analyzed by Gräfener et al. (1998) and Crowther et al. (2002). According to the latter paper, the luminosities of these stars range from $\log L=5.4$ to 5.7. Gräfener et al. (1998) obtained slightly lower values (typically by $0.2 \mathrm{dex}$ ), but used models without clumping and iron-line blanketing at that time. Compared to the average luminosity of the WC4 stars in our Galactic sample (see Table 6), it seems that their counterparts in the LMC are significantly brighter. The mass-loss rates, on the other hand, are about the same (the assumptions on clumping are identical in both works), which is obviously the reason why their line spectra are similar. One can conclude that (i) WC4 stars in the LMC have a higher current mass than Galactic stars of the same type and (ii) a higher luminosity is needed at LMC metallicity to drive the same mass loss. For probably related reasons, WC stars of later subtypes and thus larger pseudo-photospheric radii do not exist in the LMC.

\section{Conclusions}

We have analyzed 56 Galactic WC stars by comparing their line spectra to simulations from a grid of line-blanketed PoWR 
Table 6. Mean WC star parameters per subtype

\begin{tabular}{lcccccc}
\hline \hline Subtype & $\begin{array}{c}T_{*} \\
{[\mathrm{kK}]}\end{array}$ & $\begin{array}{c}v_{\infty} \\
{\left[\mathrm{km} \mathrm{s}^{-1}\right]}\end{array}$ & $\begin{array}{c}R_{*} \\
{\left[R_{\odot}\right]}\end{array}$ & $\begin{array}{c}\log \dot{M}^{a} \\
{\left[M_{\odot} / \mathrm{yr}\right]}\end{array}$ & $\begin{array}{c}\log L \\
{\left[L_{\odot}\right]}\end{array}$ & $\begin{array}{c}M_{\mathrm{WC}}{ }^{b} \\
{\left[M_{\odot}\right]}\end{array}$ \\
\hline WO2 & 200 & 5000 & 0.6 & -5.06 & 5.7 & 19 \\
WC4 & 117 & 3310 & 1.0 & -4.65 & 5.2 & 10 \\
WC5 & 83 & 2780 & 2.2 & -4.62 & 5.3 & 12 \\
WC6 & 78 & 2270 & 2.9 & -4.61 & 5.5 & 14 \\
WC7 & 71 & 2010 & 2.9 & -4.79 & 5.3 & 11 \\
WC8 & 60 & 1810 & 4.2 & -4.80 & 5.3 & 11 \\
WC9 & 44 & 1390 & 6.6 & -4.80 & 5.2 & 10 \\
\hline
\end{tabular}

Notes. ${ }^{(a)}$ Mass-loss rates are calculated with a volume-filling factor of $f_{\mathrm{V}}=0.1$. ${ }^{(b)} \mathrm{WC}$ masses are calculated from luminosities using the relation of Langer (1989).

model atmospheres to derive the stellar parameters. In addition, the SED was fitted by adjusting the reddening and scaling the luminosity to match flux-calibrated spectra and photometric measurements. For the first time, a clear correlation between the spectroscopic subtype and the stellar parameters (Table 6) has been found.

Our results also show that for single WC stars the subtypes are aligned along a strip in the $\log T_{*}-\log R_{\mathrm{t}}$-plane with the WC9 stars having a slight offset. Moreover, in the $\dot{M}$ - $L$-plane, we obtained an $\dot{M}$ - $L$-relation for WC stars in the form of $\dot{M} \propto L^{0.8}$, which is in good agreement with Nugis \& Lamers (2000).

For the chemical composition, the helium-to-carbon ratio seems to be rather uniform within the WC subclass (about 55:40 by mass). The oxygen abundance is of the order of 5\%, but might be slightly higher for the earliest subtypes and is about $30 \%$ for the WO stars.

For the terminal wind velocity, we used values from Prinja et al. (1990) and Niedzielski \& Skorzynski (2002) when available and scaled them for the remaining stars before calculating the resulting stellar parameters. Table 6 reveals a significant increase in the mean value of $v_{\infty}$ per subtype. Starting with values of about $v_{\infty} \approx 1400 \mathrm{~km} \mathrm{~s}^{-1}$ for the WC9 stars with some of them having even smaller values (close to $1000 \mathrm{~km} \mathrm{~s}^{-1}$ ), $v_{\infty}$ quickly increases for earlier subtypes, ending up as $\approx 3300 \mathrm{~km} \mathrm{~s}^{-1}$ for WC4 stars. The masses obtained from the luminosity via the relation of Langer (1989) seem to roughly increase from late to early subtypes, but these values have to be interpreted with care. The underlying mass-luminosity relation is indeed independent of the scenario that led to the WR stage.

The stars marked as WC9d display a significant excess even in the near-infrared, a finding that remains unexplained. Some of these stars might be part of a binary system. We obtained similar parameters as in our a binary pseudo fit of the well-known binary system WR 104 for some stars that are thought to be single stars. However, Williams et al. (2005) found indications that not all WC9d-stars might have a companion.

The WO stars WR 102 and WR 142 (both of WO2 subtype) differ significantly from the WC stars. They contain much more oxygen $\left(X_{\mathrm{O}} \approx 30 \%\right)$ and have both much higher temperatures $\left(T_{*} \approx 200 \mathrm{kK}\right)$, and extremely fast winds with $v_{\infty} \approx 5000 \mathrm{~km} \mathrm{~s}^{-1}$. They are very compact objects with masses higher than those of the WC stars and stellar radii smaller than $1 R_{\odot}$. Their round emission-line profiles indicate that these stars are rapid rotators.

The WN/WC stars are WN stars with an enhanced carbon fraction that have WN-type spectra. They are fitted with special models that reflect these compositions, revealing that their basic parameters $\left(T_{*}, R_{\mathrm{t}}\right)$ are already close to those of the
WC sequence. The star WR 126 is sometimes thrown into this scheme because it is classified as WC/WN. In all cases, WR 126 does not fit into any of the former groups, and seems to be an exception that has either its own unique history or is a so far undetected binary system, this last possibility being supported by its proximity to the binary-pseudo-fit region in the $\log T_{*^{-}}$ $\log R_{\mathrm{t}}$-plane.

The obtained positions of the WC stars in the HRD challenge the standard scenario that WC stars generally arise from very massive stars. Our results instead indicate that WC stars mostly originate from an intermediate-high mass range with initial masses of between $20 M_{\odot}$ and $40 M_{\odot}$ or maybe $50 M_{\odot}$. This suggests that they are descendants of post-RSG or - for the higher masses - LBV stars, which have lost enough of their hydrogen envelope that they directly enter the WNE stage before they eventually become WC stars.

Although rotation decreases the minimum mass that is required to reach the WR stage, it seems that the scenarios without initial rotation provide a closer fit to the observations. For the WC stars, the tracks of Vanbeveren et al. (1998) did not include rotation, but instead higher RSG mass-loss rates. These tracks are in much better agreement with our WC results than the Geneva tracks. The positions of the WO stars in the HRD are closer to the non-rotational Geneva tracks of Meynet \& Maeder (2003), which appears to contradict the afore mentioned results for their line profiles, but it could simply indicate that fast rotation occurs only during the later stages of evolution.

The evolutionary status of the WC9 stars is quite obscure, at least if they are single stars. They might originate from stars with initial masses of around $20 M_{\odot}$, but their evolutionary fate is completely unclear. As a first approach, one could imagine that they evolve to earlier WC subtypes, but that would have to be consistent with their increasing luminosity, as for early WC subtypes the luminosities are much higher than for the WC9 stars. Such an increase in luminosity would contradict the current assumptions of the mass-luminosity relation that correlates larger masses with larger luminosities.

The WNL stars, using the term for WN stars containing hydrogen, seem to be different from the rest of the WR stars. Their HRD positions suggest that these stars might not actually be in the phase of helium burning traditionally expected of all WR stars. They might instead simply be core-hydrogen burning stars with very high masses and mass-loss rates, making them a kind of extension of the $\mathrm{O}$ stars at the high-mass end of the main sequence. They are therefore sometimes not considered as "real" Wolf-Rayet stars. While this might seem logical in the way of using the term Wolf-Rayet stars for helium-burning stars, it is incorrect, as WR stars are not defined in terms of their core burning status but only their spectral characteristics. Nevertheless, the differences in the evolutionary status between the WNL and the other WR stars should be carefully taken into account for future stellar evolution calculations.

The WO stars, at least those of WO2 subtype, seem to differ significantly from WC stars, as we conclude from the different parameters and HRD positions. However, their evolutionary status remains unclear. As a tentative explanation, we suggest the scenarios (11) and (12). With only four known WO stars in our Galaxy, the observational basis is much worse than for either WN or WC stars, especially as our results indicate that WO stars are not descendants of WC stars. Scenario (11) probably leads only in special situations to a WO star, making WO stars such a rare subclass.

When comparing the obtained HRD positions with evolutionary models, we see a significant temperature discrepancy for 
Table 7. Suggested single-star evolution scenarios based on WN and WC analyses.

\begin{tabular}{lc}
\hline \hline$M_{\text {init }}\left[M_{\odot}\right]$ & Stellar evolution \\
\hline $8-15$ & OB $\rightarrow$ RSG $\rightarrow$ SN II-P \\
$15-20$ & OB $\rightarrow$ RSG $\rightarrow$ BSG $\rightarrow$ SN II-L \\
$20-45$ & O $\rightarrow$ RSG $\rightarrow$ WNE $\rightarrow$ WC $\rightarrow$ SN Ib/c \\
$45-60$ & O $\rightarrow$ WNL $\rightarrow$ LBV $/ \mathrm{WNE} ? \rightarrow$ WO $\rightarrow$ SN Ib/c \\
$>60$ & O $\rightarrow$ Of $/ \mathrm{WNL} \leftrightarrow$ LBV $\rightarrow$ WNL $\rightarrow$ SN IIn \\
\hline
\end{tabular}

WC5 stars and all later subtypes. A promising candidate to solve this problem is envelope inflation, as described for $\mathrm{H}$-free stars in Gräfener et al. (2012). Unlike the later subtypes, the WC4 stars and also the very different WO stars do not seem to be inflated.

A summary of the suggested single-star evolutionary scenarios for massive stars based on the discussion in Sect. 6 can be found in Table 7. As we found the upper mass limit for the WC stars to be somewhere in-between $40 M_{\odot}$ and $50 M_{\odot}$, we simply give the mean value of $45 M_{\odot}$. All of the mass limits specified in Table 7 are just rough suggestions, which are based on our results and the referenced comparisons to $\mathrm{SN}$ statistics and stellar evolution models.

Detailed stellar evolution models, including higher RSG mass-loss rates and rotation, would enable us to check whether our suggested evolutionary scenarios can be numerically reproduced. More reliable distance determinations for the Galactic WR stars would help to verify the low luminosities obtained in this work. Detailed analyses of WC and WO stars in other galaxies would help us to circumvent the distance problems and enhance our proposed scenario in order to check how it depends on metallicity.

Both the WC and WO star parameters can provide constraints on the wind-driving mechanisms and the WC massloss rates can influence the chemical evolution of our Galaxy. Furthermore, WC and WO stars are keystones in the present theories for the evolution of massive stars. In the past years, our picture of massive stars has changed and new evolutionary scenarios have been proposed. We hope that our results and suggestions can contribute to these discussions and help us to better understand the origin and evolution not only of the WC stars, but of Wolf-Rayet stars in general.

PoWR model remark: The model grid used for the Galactic WC stars will be published on our PoWR website ${ }^{2}$ with the model files being ready-to-use. The interface will allow you to select and view the basic model parameters before downloading the model itself.

Acknowledgements. We acknowledge the helpful suggestions of the anonymous referee. We would like to thank Dany Vanbeveren for providing us with additional evolutionary track material. This research has made use of the SIMBAD database, operated at CDS, Strasbourg, France. Most UV spectra used in this work are based on INES data from the IUE satellite. This publication makes use of data products from the Two Micron All Sky Survey, which is a joint project of the University of Massachusetts and the Infrared Processing and Analysis Center/California Institute of Technology, funded by the National Aeronautics and Space Administration and the National Science Foundation.

\section{References}

Ardila, D. R., van Dyk, S. D., Makowiecki, W., et al. 2010, VizieR Online Data Catalog, 219, 10301

Arnal, E. M. 1992, A\&A, 254, 305

Bakker, R., \& Thé, P. S. 1983, A\&AS, 52, 27

\footnotetext{
2 http://wWw .astro.physik. uni-potsdam.de/PoWR.html
}

Barlow, M. J., \& Hummer, D. G. 1982, in Wolf-Rayet Stars: Observations, Physics, Evolution, ed. C. W. H. De Loore, \& A. J. Willis (The Netherlands: Springer), IAU Symp., 99, 387

Barniske, A., Hamann, W., \& Gräfener, G. 2006, in Stellar Evolution at Low Metallicity: Mass Loss, Explosions, Cosmology, ed. H. J. G. L. M. Lamers, N. Langer, T. Nugis, \& K. Annuk (San Francisco: ASP), ASP Conf. Ser., 353, 243

Cardelli, J. A., Clayton, G. C., \& Mathis, J. S. 1989, ApJ, 345, 245

Chapman, J. M., Leitherer, C., Koribalski, B., Bouter, R., \& Storey, M. 1999, ApJ, 518, 890

Cherepashchuk, A. M., \& Karetnikov, V. G. 2003, Astron. Rep., 47, 38

Chu, Y., \& Treffers, R. R. 1981, ApJ, 250, 615

Conti, P. S. 1979, in Mass Loss and Evolution of O-Type Stars, ed. P. S. Conti, \& C. W. H. De Loore (Springer Netherlands), IAU Symp., 83, 431

Conti, P. S., \& Vacca, W. D. 1990, AJ, 100, 431

Crowther, P., \& Walborn, N. 2011, MNRAS, 416, 1311

Crowther, P. A., De Marco, O., \& Barlow, M. J. 1998, MNRAS, 296, 367

Crowther, P. A., Dessart, L., Hillier, D. J., Abbott, J. B., \& Fullerton, A. W. 2002, A\&A, 392, 653

Crowther, P. A., Morris, P. W., \& Smith, J. D. 2006, ApJ, 636, 1033

de Jager, C., Nieuwenhuijzen, H., \& van der Hucht, K. A. 1988, A\&AS, 72, 259

Dessart, L., Crowther, P. A., Hillier, D. J., et al. 2000, MNRAS, 315, 407

Dopita, M. A., McGregor, P. J., Rawlings, S. J., \& Lozinskaia, T. A. 1990, ApJ, 351,563

Dougherty, S. M., Williams, P. M., van der Hucht, K. A., Bode, M. F., \& Davis, R. J. 1996, MNRAS, 280, 963

Dougherty, S. M., Williams, P. M., \& Pollacco, D. L. 2000, MNRAS, 316, 143

Drew, J. E., Barlow, M. J., Unruh, Y. C., et al. 2004, MNRAS, 351, 206

Dwarkadas, V. V. 2011, MNRAS, 412, 1639

Egan, M. P., Price, S. D., Kraemer, K. E., et al. 2003, VizieR Online Data Catalog, 5114, 0

Fitzpatrick, E. L. 1999, PASP, 111, 63

Gal-Yam, A., \& Leonard, D. C. 2009, Nature, 458, 865

Gal-Yam, A., Leonard, D. C., Fox, D. B., et al. 2007, ApJ, 656, 372

Gamen, R. C., \& Niemela, V. S. 2003, in A Massive Star Odyssey: From Main Sequence to Supernova, ed. K. van der Hucht, A. Herrero, \& C. Esteban (San Francisco: ASP), IAU Symp., 212, 184

Garmany, C. D., \& Stencel, R. E. 1992, A\&AS, 94, 211

Gräfener, G., \& Hamann, W. 2005, A\&A, 432, 633

Gräfener, G., Hamann, W., Hillier, D. J., \& Koesterke, L. 1998, A\&A, 329, 190

Gräfener, G., Koesterke, L., \& Hamann, W. 2002, A\&A, 387, 244

Gräfener, G., Owocki, S. P., \& Vink, J. S. 2012, A\&A, 538, A40

Hamann, W., \& Gräfener, G. 2004, A\&A, 427, 697

Hamann, W., \& Koesterke, L. 1998, A\&A, 335, 1003

Hamann, W., \& Schmutz, W. 1987, A\&A, 174, 173

Hamann, W., Leuenhagen, U., Koesterke, L., \& Wessolowski, U. 1992, A\&A, 255,200

Hamann, W. R., Koesterke, L., \& Wessolowski, U. 1993, A\&A, 274, 397

Hamann, W., Koesterke, L., \& Wessolowski, U. 1995, A\&A, 299, 151

Hamann, W., Gräfener, G., \& Koesterke, L. 2003, in A Massive Star Odyssey: From Main Sequence to Supernova, ed. K. van der Hucht, A. Herrero, \& C. Esteban (San Francisco: ASP), IAU Symp., 212, 198

Hamann, W., Gräfener, G., \& Liermann, A. 2006, A\&A, 457, 1015

Hamann, W., Feldmeier, A., \& Oskinova, L. M. 2008, Clumping in hot-star winds (Universitätsverlag Potsdam)

Hillier, D. J. 1987, ApJS, 63, 947

Hillier, D. J. 1989, ApJ, 347, 392

Hillier, D. J., \& Miller, D. L. 1998, ApJ, 496, 407

Hillier, D. J., \& Miller, D. L. 1999, ApJ, 519, 354

Ishii, M., Ueno, M., \& Kato, M. 1999, PASJ, 51, 417

Knödlseder, J., Cerviño, M., Le Duigou, J.-M., et al. 2002, A\&A, 390, 945

Koesterke, L., \& Hamann, W. 1995, A\&A, 299, 503

Koesterke, L., Hamann, W., \& Wessolowski, U. 1992, A\&A, 261, 535

Kotak, R., \& Vink, J. S. 2006, A\&A, 460, L5

Langer, N. 1989, A\&A, 210, 93

Langer, N., Hamann, W., Lennon, M., et al. 1994, A\&A, 290, 819

Liermann, A., Hamann, W.-R., Oskinova, L. M., Todt, H., \& Butler, K. 2010, A\&A, 524, A82

Linder, N., Rauw, G., Manfroid, J., et al. 2009, A\&A, 495, 231

Lortet, M.-C., Georgelin, Y. P., \& Georgelin, Y. M. 1987, A\&A, 180, 65

Lundström, I., \& Stenholm, B. 1984, A\&AS, 58, 163

Lynga, G. 1968, The Observatory, 88, 20

Maeder, A., \& Meynet, G. 2011, Rev. Mod. Phys., accepted [arXiv: 1109.6171]

Martín, M. C., Cappa, C. E., \& Testori, J. C. 2007, Rev. Mex. Astron. Astrofis., 43, 243

Martins, F., Hillier, D. J., Paumard, T., et al. 2008, A\&A, 478, 219 
Massey, P., \& Johnson, J. 1993, AJ, 105, 980

Massey, P., DeGioia-Eastwood, K., \& Waterhouse, E. 2001, AJ, 121, 1050

Meynet, G., \& Maeder, A. 2003, A\&A, 404, 975

Meynet, G., Georgy, C., Hirschi, R., et al. 2011, Bull. Soc. Roy. Sci. Liege, 80,266

Miller, G. J., \& Chu, Y. 1993, ApJS, 85, 137

Moffat, A. F. J., \& Vogt, N. 1973, A\&A, 23, 317

Moffat, A. F. J., Fitzgerald, M. P., \& Jackson, P. D. 1977, ApJ, 215, 106

Nazé, Y., Rauw, G., \& Manfroid, J. 2008, A\&A, 483, 171

Niedzielski, A., \& Skorzynski, W. 2002, Acta Astron., 52, 81

Niemela, V. S. 1991, in Wolf-Rayet Stars and Interrelations with Other Massive

Stars in Galaxies, ed. K. A. van der Hucht, \& B. Hidayat (The Netherlands: Springer), IAU Symp., 143, 201

Nugis, T., \& Lamers, H. J. G. L. M. 2000, A\&A, 360, 227

Oskinova, L. M., \& Hamann, W. 2008, MNRAS, 390, L78

Oskinova, L. M., Hamann, W., Feldmeier, A., Ignace, R., \& Chu, Y. 2009, ApJ, 693, L44

Pasemann, D., Rühling, U., \& Hamann, W. 2011, Bull. Soc. Roy. Sci. Liege, 80, 180

Petrovic, J., Pols, O., \& Langer, N. 2006, A\&A, 450, 219

Prinja, R. K., Barlow, M. J., \& Howarth, I. D. 1990, ApJ, 361, 607

Przybilla, N., Firnstein, M., Nieva, M. F., Meynet, G., \& Maeder, A. 2010, A\&A, 517, A38

Radoslavova, T. 1989, Astron. Nachr., 310, 223

Rühling, U. 2008, Diplomarbeit, Universität Potsdam

Rustamov, D. N., \& Cherepashchuk, A. M. 1989, AZh, 66, 67

Schmutz, W., Hamann, W., \& Wessolowski, U. 1989, A\&A, 210, 236

Seaton, M. J. 1979, MNRAS, 187, 73P

Shorlin, S. L., Turner, D. G., \& Pedreros, M. H. 2004, PASP, 116, 170

Shylaja, B. S. 1990, Ap\&SS, 164, 63

Skrutskie, M. F., Cutri, R. M., Stiening, R., et al. 2006, AJ, 131, 1163

Smartt, S. J. 2009, ARA\&A, 47, 63

Smartt, S. J., Eldridge, J. J., Crockett, R. M., \& Maund, J. R. 2009, MNRAS, 395,1409

Smith, L. F. 1968, MNRAS, 140, 409

Smith, N. 2006, ApJ, 644, 1151

Smith, N. 2008, in Massive stars as cosmic engines, ed. F. Bresolin, P. A. Crowther, \& J. Puls (Cambridge University Press), IAU Symp., 250, 193
Smith, N., \& Conti, P. S. 2008, ApJ, 679, 1467

Smith, L. F., Shara, M. M., \& Moffat, A. F. J. 1990, ApJ, 358, 229

Smith, N., Li, W., Filippenko, A. V., \& Chornock, R. 2011, MNRAS, 412, 1522

Thé, P. S., \& Stokes, N. 1970, A\&A, 5, 298

Torres, A. V., \& Massey, P. 1987, ApJS, 65, 459

Trundle, C., Kotak, R., Vink, J. S., \& Meikle, W. P. S. 2008, A\&A, 483, L47

Turner, D. G. 1996, AJ, 111, 828

Turner, D. G., \& Forbes, D. 1982, PASP, 94, 789

Turner, D. G., Rohanizadegan, M., Berdnikov, L. N., \& Pastukhova, E. N. 2006, PASP, 118,1533

van der Hucht, K. A. 2001, New Astron. Rev., 45, 135

van der Hucht, K. A. 2006, A\&A, 458, 453

van Genderen, A. M., Verheijen, M. A. W., van der Hucht, K. A., et al. 1991, in Wolf-Rayet Stars and Interrelations with Other Massive Stars in Galaxies, ed. K. A. van der Hucht, \& B. Hidayat (The Netherlands: Springer), IAU Symp., 143,129

van Loon, J. T., Cioni, M.-R. L., Zijlstra, A. A., \& Loup, C. 2005, A\&A, 438, 273

Vanbeveren, D., De Donder, E., van Bever, J., van Rensbergen, W., \& De Loore, C. 1998, New Astron., 3, 443

Vanbeveren, D., Van Bever, J., \& Belkus, H. 2007, ApJ, 662, L107

Varricatt, W. P., \& Ashok, N. M. 2006, MNRAS, 365, 127

Vazquez, R. A., Will, J.-M., Prado, P., \& Feinstein, A. 1995, A\&AS, 111, 85

Vázquez, R. A., Baume, G. L., Feinstein, C., Nuñez, J. A., \& Vergne, M. M. 2005, A\&A, 430, 471

Wallace, D. J., Moffat, A. F. J., \& Shara, M. M. 2002, in Interacting Winds from Massive Stars, ed. A. F. J. Moffat, \& N. St-Louis (San Francisco: ASP), ASP Conf. Ser., 260, 407

Wallace, D. J., Gies, D. R., Moffat, A. F. J., Shara, M. M., \& Niemela, V. S. 2005, AJ, 130, 126

Williams, P. M., van der Hucht, K. A., \& The, P. S. 1987, A\&A, 182, 91

Williams, P. M., van der Hucht, K. A., Kidger, M. R., Geballe, T. R., \& Bouchet, P. 1994, MNRAS, 266, 247

Williams, P. M., Kidger, M. R., van der Hucht, K. A., et al. 2001, MNRAS, 324, 156

Williams, P. M., van der Hucht, K. A., \& Rauw, G. 2005, in Massive Stars and High-Energy Emission in OB Associations, ed. G. Rauw, Y. Nazé, \& R. E. Blomme Gosset, 65 


\section{Appendix A: Comments on individual stars}

In this appendix we compile some additional information about individual stars of our sample, especially their distance and binary status:

WR 4 is a WC5 star that is listed as SB1 in van der Hucht (2001) owing to its short-periodic photometric variability measured by Rustamov \& Cherepashchuk (1989), but it displays neither diluted emission nor absorption lines from a possible companion. Smith et al. (1990) discussed whether WR 4 belongs to an $\mathrm{H}_{\mathrm{I}}$ bubble, for which they estimate a distance of between $1.6 \mathrm{kpc}$ and $2.73 \mathrm{kpc}$ depending on the method. The smaller value is close to our spectroscopic distance of $1.64 \mathrm{kpc}$, based on the $M_{v}$ calibration.

WR 8 is classified as WN7/WCE. Its spectrum resembles a WN7 type star, but with stronger carbon lines than usual. Niemela (1991) measured large amplitude radial-velocity variations with a possible period of $38.4 \mathrm{~d}$, where the carbon and nitrogen lines are in antiphase. This is indicative of a $\mathrm{WN}+\mathrm{WC}$ binary system. We attempted to reproduce the spectrum using a single-star model, which resembles a hydrogen-free WN star with enhanced carbon, but failed to obtain a convincing fit (Fig. B.3). Lundström \& Stenholm (1984) estimated the modulus to be D.M. $=12.7 \mathrm{mag}$ based on a membership of the Anon Pup a association.

WR 14 is a WC7 star, for which photometric variability has been reported by various authors, and discussed by Shylaja (1990) in terms of a possible compact companion. It displays non-thermal radio emission (Chapman et al. 1999), which can normally be attributed to colliding winds in binary systems. We found that its spectrum can be reproduced with a typical WC model for single stars, and conclude that there is at least no bright companion that dilutes the WC emission lines. Lundström \& Stenholm (1984) identified the star as a member of Anon Vel a, estimating a distance of D.M. = $11.5 \mathrm{mag}$.

WR 15 has the spectral type WC6. From the width of its lines, we estimated a terminal wind velocity of $2600 \mathrm{~km} \mathrm{~s}^{-1}$, slightly higher values than typical for this subtype. The distance of D.M. = $12.0 \mathrm{mag}$ is based on the possible membership of Anon Vel b suggested by Lundström \& Stenholm (1984). We prefer to used this distance method over that used to calculate the spectroscopic parallaxes obtained by Conti \& Vacca (1990), who derived a value of D.M. = $11.28 \mathrm{mag}$ for WR 15 .

WR 23 is another a WC6 star and belongs to the Car OB1 association, for which Smith (2006) obtained a distance modulus of D.M. = $11.8 \mathrm{mag}$ for the Homunculus nebula around $\eta$ Car. Earlier distance calculations for Car OB1 were D.M. = 12.55 mag (Massey \& Johnson 1993) and 12.1 mag (Lundström \& Stenholm 1984).

WR 26 is classified as WN7/WCE. The spectrum contains a very strong $\mathrm{C}$ IV $5808 \AA$ line, which requires a model with about a $20 \%$ carbon mass fraction to be reproduced. The fit to our single-star model is satisfactory. The high carbon abundance might indicate that WR 26 is undergoing a transition from a WN to a WC star.

WR 33 has the spectral type WC5. The UV spectrum is indicative of a high wind velocity of about $3000 \mathrm{~km} \mathrm{~s}^{-1}$, but otherwise looks normal. The optical spectrum (from Torres \& Massey 1987) shows very broad emission features between $4000 \AA$ and $4600 \AA$ that cannot be reproduced by any of our models.

WR 38 displays a WC4 spectrum. Our fit is not very consistent, possibly indicating that the lines are diluted by a companion's continuum. From a weak cluster around WR 38, Shorlin et al. (2004) derived a very large distance of D.M. = $15.8 \mathrm{mag}$, which would imply a very high luminosity of this star. In contrast, Wallace et al. (2005) obtained a distance modulus of $15.0_{-1.0}^{+1.5} \mathrm{mag}$ from the HST photometry of the same cluster. Our photometric distance, based on the subtype calibration, yields D.M. = $15.1 \mathrm{mag}$, in perfect agreement with the latter work.

WR 39, classified as WC7+OB? (van der Hucht 2001), is an interesting test case for the "diluted emission-line" binarity criterion. When we analyze its line spectrum assuming that the star is single, the obtained parameters place the star distinctly above the sequence of single WC stars in the $\log T_{*}-\log R_{\mathrm{t}^{-}}$ plane (see Fig. 6). Moreover, WR 39 shows non-thermal radio emission (Chapman et al. 1999), which is normally attributed to colliding winds in binary systems. We therefore conclude that WR 39 is indeed most likely a binary.

WR 45 is a WC6 star. Unfortunately, the only spectrum available to us is limited to $3410-4730 \AA$ and does not cover many diagnostic lines. Therefore, the parameters we obtained for this star are uncertain.

WR 50 is a double-lined spectroscopic binary WC7+OB (van der Hucht 2001) with a light-curve period of $1 \mathrm{~d}$ (van Genderen et al. 1991). We analyzed its spectrum as if it were from a single star, and obtained parameters that are obviously affected by the d.e.l. effect (cf. Fig. 6). The distance modulus is D.M. = 12.8 mag according to Vázquez et al. (2005).

WR 52 is one of only five known Galactic WC4 stars, and does not show indications of binarity. The spectral fit Fig. B.16) requires models with higher oxygen abundances $(10-15 \%$ by mass) than we used for our standard WC grid (5\%). This agrees with our findings of higher oxygen abundances for WC4 and WO stars. As discussed by Chu \& Treffers (1981), a kinematic distance of $2 \mathrm{kpc}$ and a photometric distance of $4 \mathrm{kpc}$ were derived from the associated nebula. From our subtype calibration, we obtained a distance modulus of D.M. $=12.67 \mathrm{mag}$, corresponding to $3.4 \mathrm{kpc}$.

WR 53 is classified as WC8 with the " $\mathrm{d}$ " indicating persistent dust. In contrast to the WC9 stars, dust formation is uncommon for this subtype, and might indicate colliding winds. The fit to our models, calculated with the full $\mathrm{C}_{\text {II }}$ ion, remains remarkably poor (Fig. B.17) for unknown reasons. Martín et al. (2007) found an expanding $\mathrm{H}_{\mathrm{I}}$ shell probably associated with this star, encircling an optical emission nebula, and estimated the distance of the shell to be $4 \pm 1 \mathrm{kpc}$. From our subtype calibration, we obtained $2.9 \mathrm{kpc}$.

WR 58 is a transition-type star, classified as WN4/WC. For this star, a carbon mass fraction of $0.1 \%$ is enough to reproduce the C IV 5808 A-line.

WR 59 is classified as WC9d. The star might be a binary, as Williams et al. (2005) found Balmer absorption features by comparing the spectral lines to those of a non-dusty WC9 star.

WR 64 is classified as WC7. The only spectrum available to us is that of Torres \& Massey (1987) and covers only a short wavelength range, making our analysis less precise.

WR 65, classified as WC9d, shows variable X-ray emission. According to Oskinova \& Hamann (2008), the emission originates from the wind-wind collision in a massive binary system, and the variability is caused by the different absorption columns along the orbit. Williams et al. (2005) detected absorption features from the Balmer lines of hydrogen. We analyzed the spectrum as if it were from a single WC star and found no indication of a composite nature. However, if WR 65 were a member of the Cir OB1 association as suggested by Lortet et al. (1987), it would be by far the brightest WC star in our sample $\left(M_{v}=-7.02 \mathrm{mag}\right.$ adopting a distance modulus from Turner 1996 of $12.57 \mathrm{mag}$ ). 
As this seems unlikely, we conclude that WR 65 is located in the foreground of Cir OB1, or it has a companion that contributes significantly to its total brightness. In any case, we cannot employ this star for our $M_{v}$ versus subtype calibration.

WR 68 is of subtype WC7 and a possible member of the Cir OB1 association. Turner (1996) deduced a distance of D.M. $=12.57 \mathrm{mag}$, based on the cluster Pismis 20, which is part of Cir OB1. Adopting this number leads to a plausible luminosity and HRD position. However, the distance of Cir OB1 has been debated in the past with distance moduli ranging from $11.58 \mathrm{mag}$ (Moffat \& Vogt 1973) to $12.8 \mathrm{mag}$ (Vazquez et al. 1995), $13.00 \mathrm{mag}$ (Lortet et al. 1987), and 13.22 mag (Lynga 1968).

WR 69 is one of the few WC9d stars for which a useful IUE spectrum exists, since the reddening for this star is relatively moderate. Williams et al. (2005) measured differences in the radial velocities between different observations, possibly indicating binarity.

WR 81 is another WC9 star, but one of the few Galactic examples without dust emission. Our line fit is remarkably much more consistent than for the dusty WC9d stars.

WR 86 is a visual binary classified as WC7 that has a B0 companion. The star is another example that helps to demonstrate which model parameters are obtained when a composite spectrum is fitted with a single-star model. We again find that the "diluted emission-line" effect places the results from this pseudo-fit in the characteristic domain of the $\log T_{*}-\log R_{\mathrm{t}}$-plane (Fig. 6).

WR 88 is classified as WC9 and does not show dust emission. Thus it resembles WR 81. Williams et al. (2005) attribute a couple of emission lines to nitrogen, and conclude that WR 88 is either a WC+WN binary or belongs to a previously unobserved transitional WN/WC9 subtype. We fitted the spectrum with our WC models, and obtained parameters that agree with those of other WC9 stars (Fig. 6). Given the poor quality of the Torres \& Massey (1987) spectrum we use, we refrain from test calculations with enhanced nitrogen abundances.

WR 90 shows non-thermal radio emission (Chapman et al. 1999), but otherwise no indications of binarity. Our single-star fit and the derived parameters are typical of its WC7 type.

WR 95 has the spectral subtype WC9d. According to Moffat et al. (1977), it belongs to the open cluster Trumpler 27 and has a distance of $2.1 \pm 0.2 \mathrm{kpc}$ implying a distance modulus of D.M. $=11.61 \mathrm{mag}$. Thé \& Stokes (1970) and Bakker \& Thé (1983) obtained lower values of $10.17 \mathrm{mag}$ and $11.09 \mathrm{mag}$, which would lead to very low luminosities.

WR 98 was originally classified as a single star of WN8/WC7 transition type. Gamen \& Niemela (2003) detected O8-9 type absorption lines superimposed on its spectrum. From the SB2 radial velocity curves, they derived the orbital elements. We tried to fit the spectrum with our single-star models. According to the WN/WC transition type of the spectrum, the best-fitting model is basically a hydrogen-free WN model with enhanced carbon ( $5 \%$ by mass). However, some spectral features cannot be reproduced by any of our single-star models, most likely because the contribution of the O-star companion cannot be neglected. We therefore consider Fig. B.34 as a "pseudo fit" that is in fact unsuitable for this composite spectrum, and omit WR 98 from our single-star analysis.

WR 102, also known as Sand 4, is one of two WO stars analyzed in this work. Classified as WO2, it has the same subtype as WR 142. To reproduce its spectrum, we needed to use models with an enhanced oxygen abundance and very high wind velocities. Moreover, the observed emission line profiles of WR 142 have a round top, which differs from the Gaussian-like shape usually encountered in WR spectra. Our models can only reproduce this round shape when we convolve the synthetic spectrum for rotational broadening with a $v \sin i$ of about $1000 \mathrm{~km} \mathrm{~s}^{-1}$. (For wind spectra, flux convolution is only a rough approximation to account for rotation.) We note that the WO stars are very compact, since their radius is smaller than the solar radius. Interestingly, the same effect has also been found for the most compact WN star in our Galaxy, the WN2 star WR 2 (Hamann et al. 2006). This rapidly rotating, bare, and compact stellar core is certainly a gamma-ray burst candidate. For the distance of WR 102, we adopt $3 \pm 1 \mathrm{kpc}$ from Dopita et al. (1990), which is based on the nebula G2.4+1.4 that was originally classified as a supernova remnant, but is now considered to be stellar ejecta from WR 102 despite this star not being located in its center. Drew et al. (2004) calculated a distance of $4.6 \mathrm{kpc}$ based on an IR photometry scaling relative to WR 142 . We did not use this distance as it does not rely on an independent measurement. Using a value of $4.6 \mathrm{kpc}$ would infer an extremely high luminosity of $\log L / L_{\odot}=6.1$.

WR 104 is well known for its pinwheel nebula. It is a binary system consisting of a WC9d and a B0.5V star. The latter is visually brighter than the WC star (Williams et al. 1987). A third, fainter component was resolved with HST (Wallace et al. 2002), hence the WC emission lines are expected to be "diluted". Nevertheless, an acceptable fit can still be achieved for WR 104 with a single-star model that has quite "typical" WC9 parameters (cf. Fig. 6). The distance (D.M. = $11.0 \mathrm{mag}$ ) is adopted from Lundström \& Stenholm (1984), who assigned WR 104 as a possible member to the association Sgr OB1.

WR 111 is a prototypical WC5 star, which has been frequently studied. Gräfener et al. (2002) basically used the same models as the present study, and therefore obtained similar results. Gräfener \& Hamann (2005) constructed a hydrodynamically consistent model for WR 111, thus showed for the first time that WC winds can be explained in terms of radiation-driven mass loss. Their model provided a more consistent fit of the line spectrum than the semi-empirical models used in the present paper. The hydrodynamical model of Gräfener \& Hamann (2005) has a much higher stellar temperature $T_{*}=140 \mathrm{kK}$ than our present study $(89 \mathrm{kK})$. This mainly reflects the different radial structures of the two respective models in the deepest zones of the wind - we recall that $T_{*}$ is defined as the effective temperature corresponding to the radius $R_{*}$, where the Rosseland optical depth reaches 20 (cf. Eq. (1)).

The model of Gräfener \& Hamann (2005) has nearly the same luminosity as we obtain from our empirical fit. The massloss rate of the hydrodynamically consistent model $(\log \dot{M}=$ $-5.14)$ is considerably lower than that of our empirical model (-4.67). This difference is mainly due to the much higher clumping contrast assumed by Gräfener \& Hamann (2005) $(D=50$ in the outer parts instead of 10 in this work). Such strong clumping is probably unrealistic, but was needed to compensate for the incomplete line opacities and achieve sufficient radiative driving. A minor part of the mass-loss rate differences is due to the terminal wind velocity, which was slightly smaller in the hydrodynamic model $\left(2050 \mathrm{~km} \mathrm{~s}^{-1}\right)$ than the value adopted in the present paper $\left(2398 \mathrm{~km} \mathrm{~s}^{-1}\right)$. For the model of Gräfener \& Hamann (2005), a much higher stellar temperature $\left(T_{*}=\right.$ $140 \mathrm{kK}$ ) was assumed than the $89 \mathrm{kK}$ given in the present paper. Different stellar radii $R_{*}$ compensate for the effect of luminosity. The similarity of the emergent spectra demonstrate again the parameter degeneracy for very dense winds discussed in Sect. 5.1. The star WR 111 is assumed to be a member of the 
Sgr OB1 association (Lundström \& Stenholm 1984) with a distance modulus of D.M. = $11.0 \mathrm{mag}$.

WR 113 is a WC8d+O8-9 binary system (Cherepashchuk \& Karetnikov 2003), which has an excess in its 2MASS K-band magnitude most probably caused by dust emission. The pseudofit of its spectrum obtained from our single-star models leads to parameters in the binary domain of the $\log T_{*}-\log R_{\mathrm{t}}$-plane (Fig. 6), obviously due to the dilution of the emission lines.

WR 114, classified as WC5, is a member of the Ser OB1 association (Lundström \& Stenholm 1984) with a distance modulus of D.M. = $11.5 \mathrm{mag}$. The spectrum was found to have diluted emission lines, and the star was therefore listed as a binary candidate in van der Hucht (2001). However, our spectral fit and the obtained parameters are normal for a single WC5 star.

WR 117 is a "dusty" WC9d star. Williams et al. (2005) did not find any evidence of an OB companion, and our single-star model also fits most features in the observed spectrum with typical parameters. The lines are significantly stronger than in other WC9 stars. The best-fitting model has a stellar temperature of $T_{*}=56 \mathrm{kK}$, which is relatively high for the WC9 subclass. The position of WR 117 in the $\log T_{*}-\log R_{\mathrm{t}}$-plane, as well as the high terminal wind velocity, are close to or maybe already in the WC8 parameter region. Some previous papers (e.g. Conti \& Vacca 1990) have indeed classified WR 117 as type WC8. Unfortunately, our available spectrum does not comprise any $\mathrm{C}$ II-lines, which would provide the criterion to distinguish the subtype WC8 from WC9.

WR 121 is another "dusty" WC9d star that is apparently single. Williams et al. (2005) could not find any evidence of an OB companion, and our single-star model fits most features in the observed spectrum with typical parameters.

WR 125 is an SB2 binary system (WC7ed+O9III, Williams et al. 1994), where the letters "ed" stand for episodic dust formation. Our pseudo fit with a single-star model gives parameters that are characteristic of composite spectra (cf. Fig. 6).

WR 126 shows a unique spectrum that differs from those of all other Wolf-Rayet subtypes. Its designation as WC5/WN indicates that it has predominantly a WC-type spectrum (albeit the emission lines are unusually weak), but also relatively strong nitrogen. We tentatively fit the spectrum with a WC-type model from our low-carbon (20\% mass fraction) grid (Fig. B.46). The N Iv lines at 7005-7031 reveal obviously a significant abundance of nitrogen, which is not included in our WC models. The mass-loss rate is much lower than those typically found for WC5 stars. A thorough spectral analysis of this transitiontype star is beyond the scope of the present paper. Following Radoslavova (1989), the star is probably a member of the Vul OB2 association, which has a distance modulus of D.M. = $13.2 \mathrm{mag}$.

WR 132 is classified as WC6 and might be associated with an H I-bubble. Based on that bubble, Arnal (1992) estimated a kinematic distance of $4.3 \mathrm{kpc}$, which we adopt for our luminosity scaling.

WR 135 is one of only four WC8 stars analyzed in this work. The star is a possible member of Cyg OB3, leading to a distance of D.M. = $11.2 \mathrm{mag}$ (Garmany \& Stencel 1992). The earlier calculations of Lundström \& Stenholm (1984) obtained a slightly higher value of $11.6 \mathrm{mag}$. WR 135 is the only WC8 single star with an independent distance estimate.

WR 137 is a binary system (WC8pd+O9) showing periodic dust ("pd") formation. Under the assumption that the O star is a main-sequence star, Williams et al. (2001) estimated a distance modulus of D.M. = $11.1 \mathrm{mag}$. The pseudo-fit with a single-star model yields parameters that are typical for composite spectra (cf. Fig. 6).

WR 142, classified as WO2, is the other WO star analyzed in this work. It has the same subtype as WR 102. The spectra of both stars are very similar, hence so are the results of their analyses. We achieved the best fit with a very high terminal wind velocity $\left(v_{\infty}=5000 \mathrm{~km} \mathrm{~s}^{-1}\right)$ and a very high stellar temperature of $T_{*}=200 \mathrm{kK}$. As for WR 102, we had to convolve the emergent spectrum with a high rotational broadening velocity of $1000 \mathrm{~km} \mathrm{~s}^{-1}$ in order to reproduce the round shape of the emission lines. Oskinova et al. (2009) detected weak but hard $\mathrm{X}$-rays from this object. These X-rays cannot be attributed to colliding stellar winds since there is apparently no companion. WR 142 is a member of the Berkeley 87 cluster. The distance of this cluster has been disputed in the past. Turner \& Forbes (1982) derived a cluster distance of $946 \pm 26 \mathrm{pc}$, which would imply $\log L / L_{\odot}=5.5$. Massey et al. (2001) and Knödlseder et al. (2002) obtained $1.58 \mathrm{kpc}$ and $1.8 \mathrm{kpc}$, raising the luminosity to 5.9 or even 6.0 for the latter distance. In our work, we used the latest value of Turner et al. (2006) of a distance of $1230 \pm 40 \mathrm{pc}$ leading to $\log L / L_{\odot}=5.7$.

WR 143 was revealed as a binary $(\mathrm{WC} 4+\mathrm{Be})$ by Varricatt \& Ashok (2006). The analysis with our single-star models yields parameters that closely fit to the WC sequence, possibly indicating that the contribution of the Be-type companion to the composite spectrum is relatively weak.

WR 144 is one of two Galactic WC4 stars that are not suspected to be binaries. According to Lundström \& Stenholm (1984), the star is a possible member of the Cyg OB2 association with a distance modulus of $11.3 \mathrm{mag}$, which we adopt for our luminosity scaling. This distance implies a luminosity of $\log L / L_{\odot}=5.22$. An alternative distance modulus of D.M. = 9.8 mag for Cyg OB2 claimed by Linder et al. (2009) would lead to an implausibly low luminosity of $\log L / L_{\odot}=4.6$.

WR 145 is of the transition type WN7/WC. Its spectrum is nicely fitted by a WN-type model with enhanced carbon. The distance modulus of $11.3 \mathrm{mag}$ is taken from Lundström \& Stenholm (1984) based on the possible membership of WR 145 in the Cyg OB2 association. The star is located in a nebula (Miller \& Chu 1993).

WR 146 is a visual binary system consisting of a WC5 star with an O8 companion. Dougherty et al. (1996) also resolved two components with a high-spatial-resolution radio observation and classified WR 146 as a colliding wind binary due to its nonthermal radio emission. Dougherty et al. (2000) performed an indepth analysis of the different types of radio emission from both components and their colliding wind region. They suggested that the companion might actually not be a single $\mathrm{O} 8 \mathrm{star}$, but a composite system itself, possibly consisting of an $\mathrm{O} 8$ and another WC star to explain the high mass-loss rate. The poor pseudo fit that can be achieved with single-star models yields parameters which are typical of binaries if the d.e.l. effect is neglected (cf. Fig. 6). Dessart et al. (2000) tried to account for the companion's continuum when they analyzed this WR star, but still arrived at parameters that are atypical of a WC5 star. WR 146 is also listed as a possible member of the Cyg OB2 association with D.M. = 11.3 mag (Lundström \& Stenholm 1984), although this membership has since been questioned. Dougherty et al. (1996) obtained D.M. = 10.4 mag, which would place WR 146 in front of Cyg OB2 and is the value that we used in our pseudo fit.

WR 154 is a WC6 single star and a possible member of the Cep OB1 association, for which Garmany \& Stencel (1992) give a distance modulus of $12.2 \mathrm{mag}$. Earlier calculations obtained 
Table B.1. Appendix overview: Galactic WC star fits.

\begin{tabular}{lccc}
\hline \hline Identifier & Page & Identifier & Page \\
\hline WR 4 & 24 & WR 86 & 52 \\
WR 5 & 25 & WR 88 & 53 \\
WR 8 & 26 & WR 90 & 54 \\
WR 13 & 27 & WR 92 & 55 \\
WR 14 & 28 & WR 95 & 56 \\
WR 15 & 29 & WR 98 & 57 \\
WR 17 & 30 & WR 102 & 58 \\
WR 23 & 31 & WR 103 & 59 \\
WR 26 & 32 & WR 104 & 60 \\
WR 27 & 33 & WR 106 & 61 \\
WR 33 & 34 & WR 111 & 62 \\
WR 38 & 35 & WR 113 & 63 \\
WR 39 & 36 & WR 114 & 64 \\
WR 45 & 37 & WR 117 & 65 \\
WR 50 & 38 & WR 119 & 66 \\
WR 52 & 39 & WR 121 & 67 \\
WR 53 & 40 & WR 125 & 68 \\
WR 56 & 41 & WR 126 & 69 \\
WR 57 & 42 & WR 132 & 70 \\
WR 58 & 43 & WR 135 & 71 \\
WR 59 & 44 & WR 137 & 72 \\
WR 60 & 45 & WR 142 & 73 \\
WR 64 & 46 & WR 143 & 74 \\
WR 65 & 47 & WR 144 & 75 \\
WR 68 & 48 & WR 145 & 76 \\
WR 69 & 49 & WR 146 & 77 \\
WR 80 & 50 & WR 150 & 78 \\
WR 81 & 51 & WR 154 & 79 \\
\hline
\end{tabular}

D.M. = 13.7 mag (Smith et al. 1990) and 12.53 mag (Conti \& Vacca 1990).

\section{Appendix B: Spectral fits}

This section lists the spectral fits of all stars analyzed in this work. For each star, a composite plot is available that consists of an SED fit (top panel, similar to Fig. 4) and several panels that show the best-fitting grid model together with the normalized UV and visual spectra when available. In all panels, the observed spectra are plotted in blue, while the model is printed in red. The flux of the SED has been decreased according to the distance and reddened with $E_{b-v}$ as given in the figures. The applied reddening law is indicated by the keywords SEATON, CARDELLI, or FITZPATRICK. The parameters $R_{V}$ is also given as the last two laws use it as a free parameter.

In most cases, the best-fitting model was taken from the standard WC grid described in Sect. 3. If a special model was required to reproduce the observed spectrum, this is clearly noted in the corresponding figure. 
WR004

$\mathrm{T}_{*}=79 \mathrm{kK} \log \mathrm{R}_{\mathrm{t}}=0.5$

WC5

$v_{\infty}=2000 \mathrm{~km} / \mathrm{s}-\mathrm{He}: \mathrm{C}: \mathrm{O}=55: 40: 5$
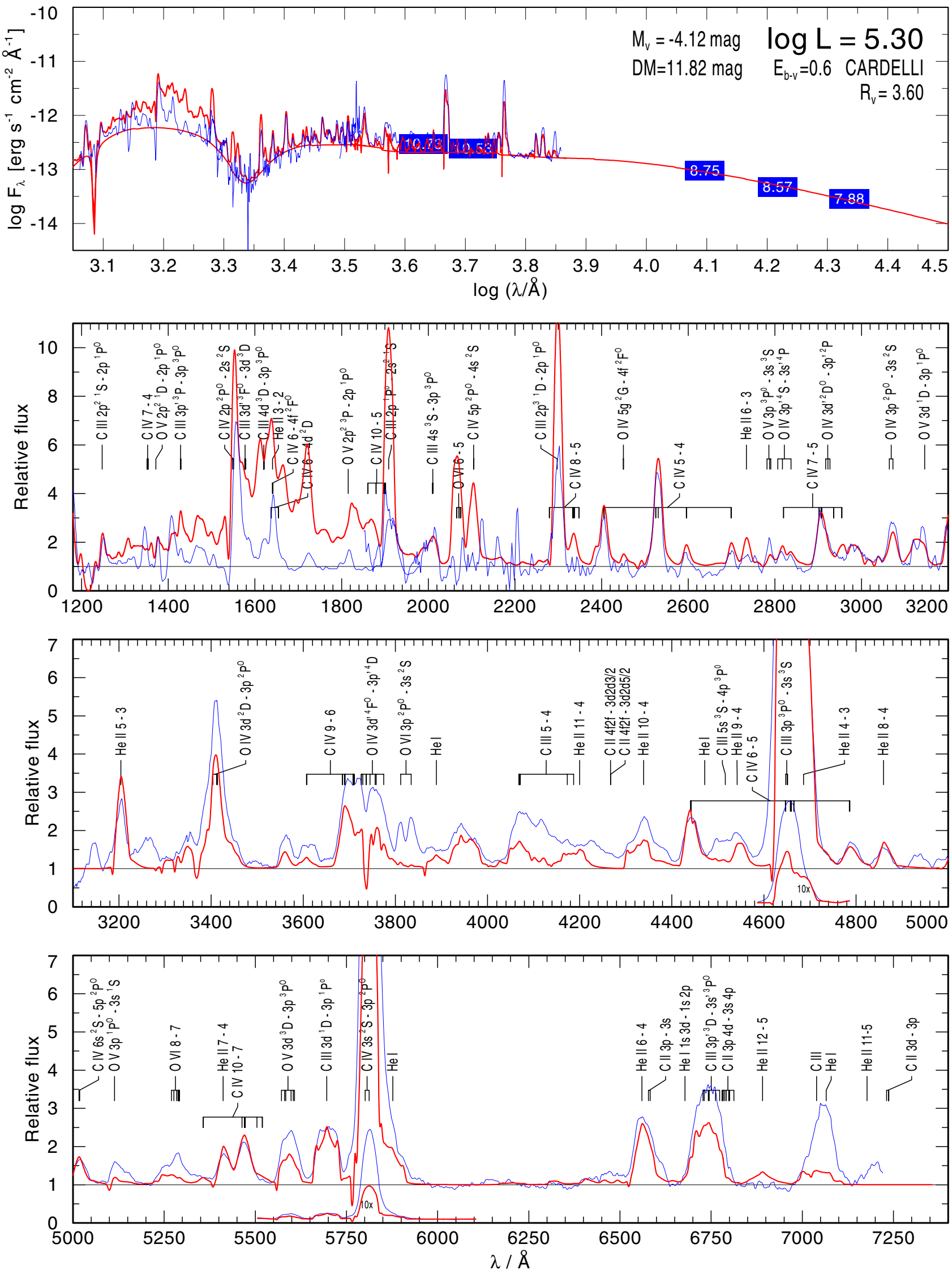

Fig. B.1. Spectral fit for WR 4. 
A. Sander et al.: The Galactic WC stars

WR005

$\mathrm{T}_{*}=79 \mathrm{kK} \log \mathrm{R}_{\mathrm{t}}=0.5$

WC6

$v_{\infty}=2000 \mathrm{~km} / \mathrm{s}-\mathrm{He}: \mathrm{C}: \mathrm{O}=55: 40: 5$
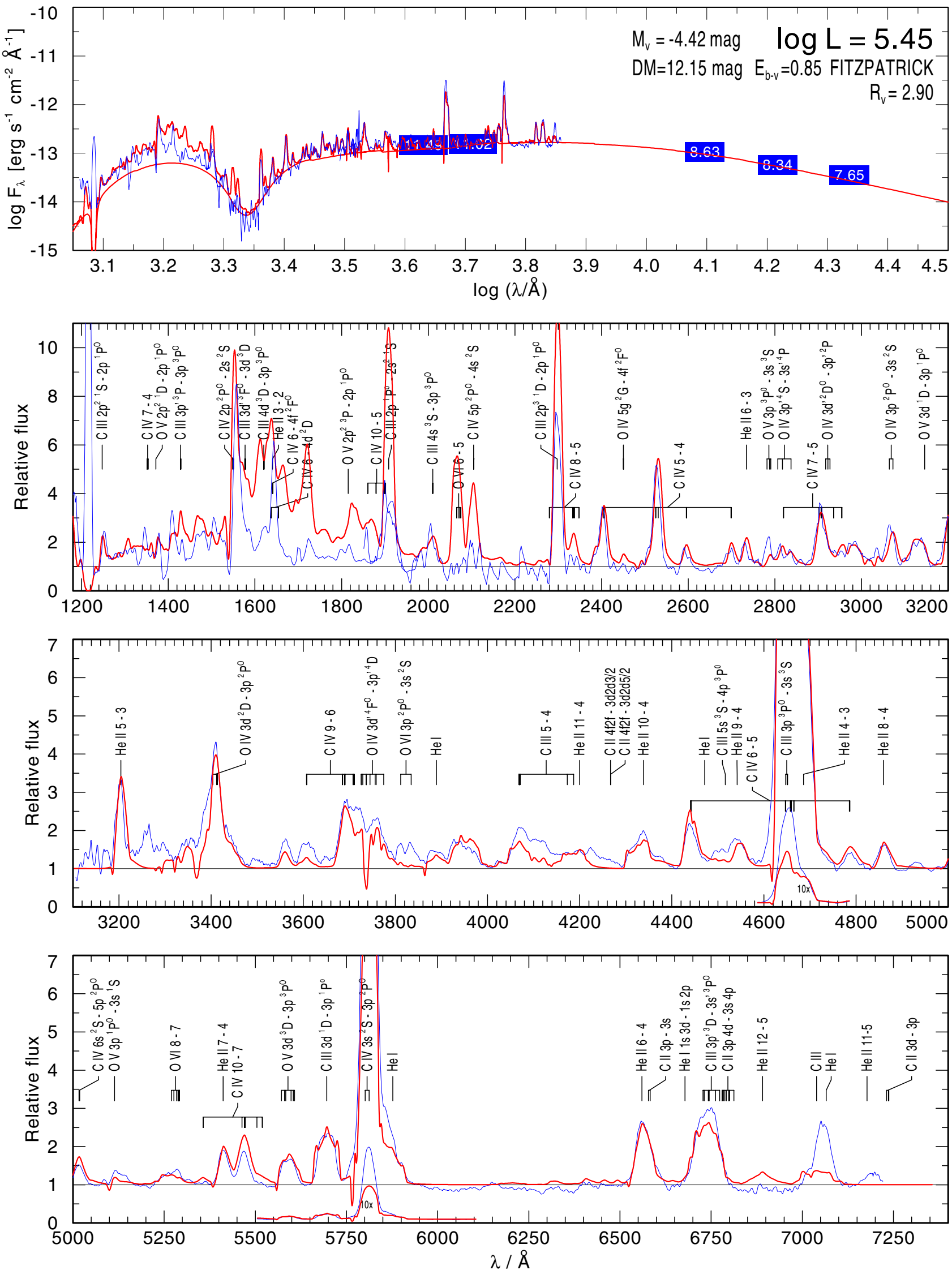

Fig. B.2. Spectral fit for WR 5. 
WR008 (Binary)

$T_{*}=56 \mathrm{kK} \log R_{t}=0.8$
WN7/WCE $v_{\infty}=1600 \mathrm{~km} / \mathrm{s}-\mathrm{He}: \mathrm{C}: \mathrm{N}=95.5: 5: 1.5$
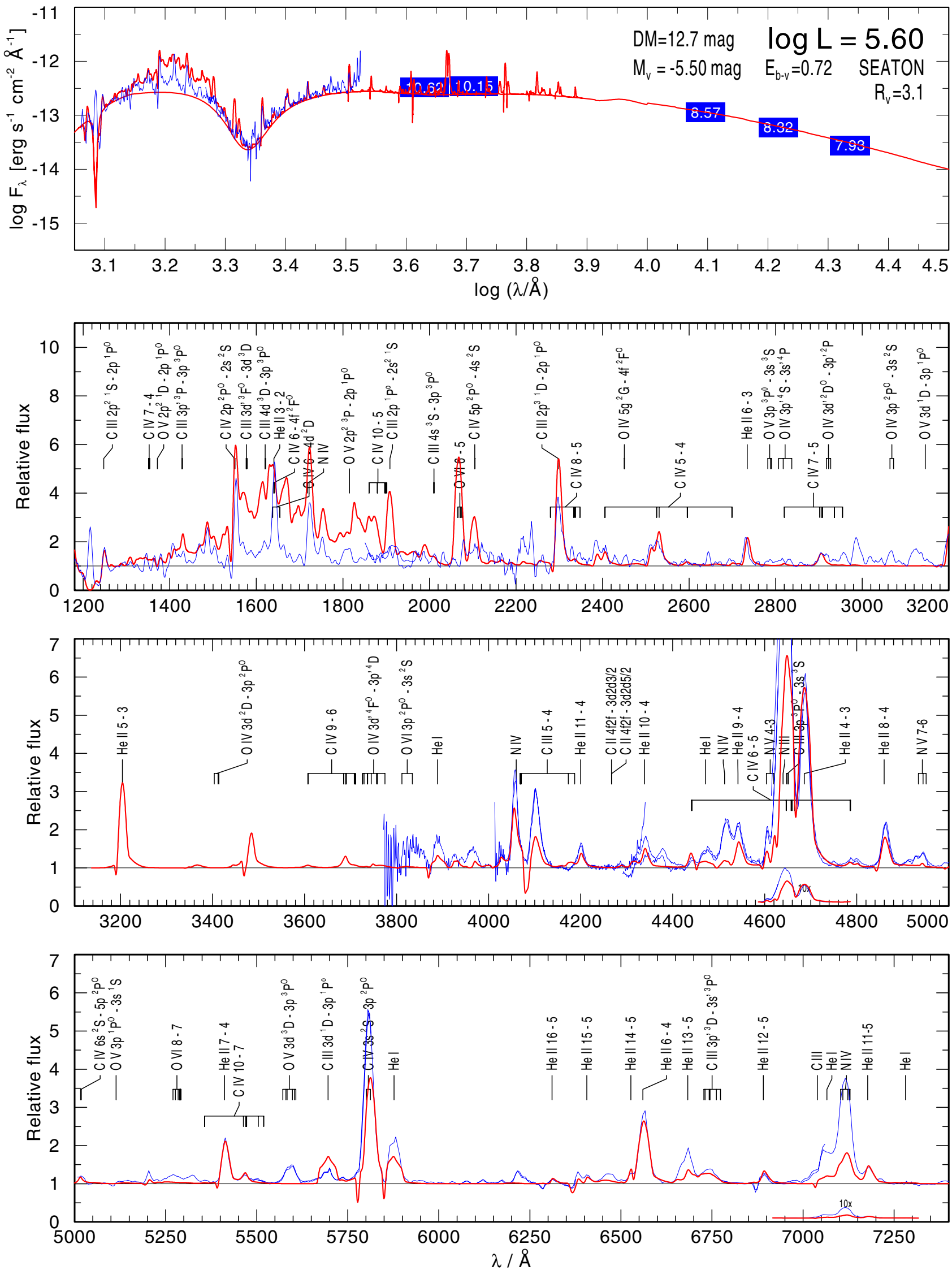

Fig. B.3. Spectral pseudo fit for WR 8, model did not contain oxygen. 
WR013

$T_{*}=79 \mathrm{kK} \log R_{t}=0.5$

WC6 $v_{\infty}=2000 \mathrm{~km} / \mathrm{s}-\mathrm{He}: \mathrm{C}: \mathrm{O}=55: 40: 5$
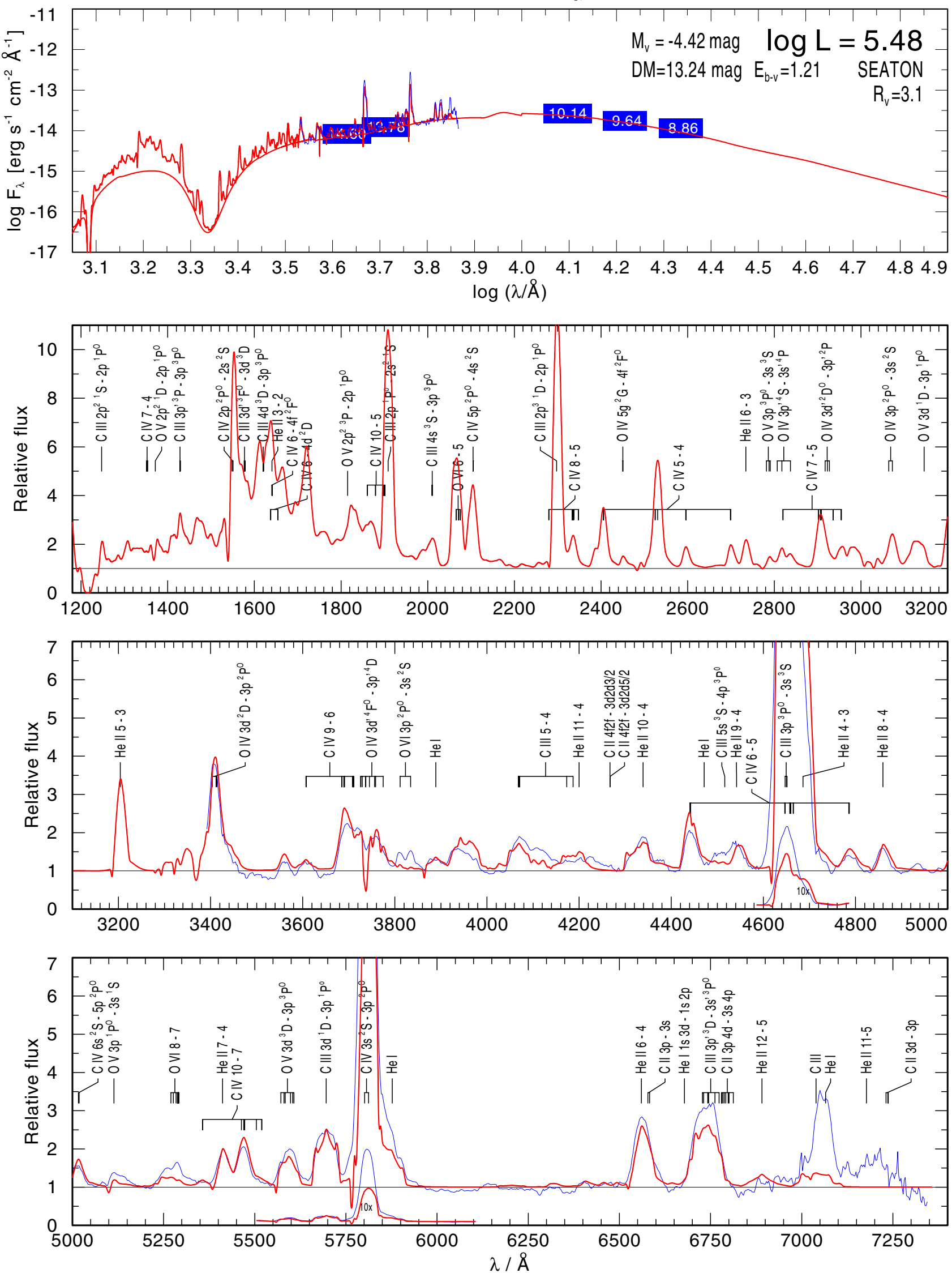

Fig. B.4. Spectral fit for WR 13. 
WR014

$T_{*}=71 \mathrm{kK} \log R_{t}=0.6$
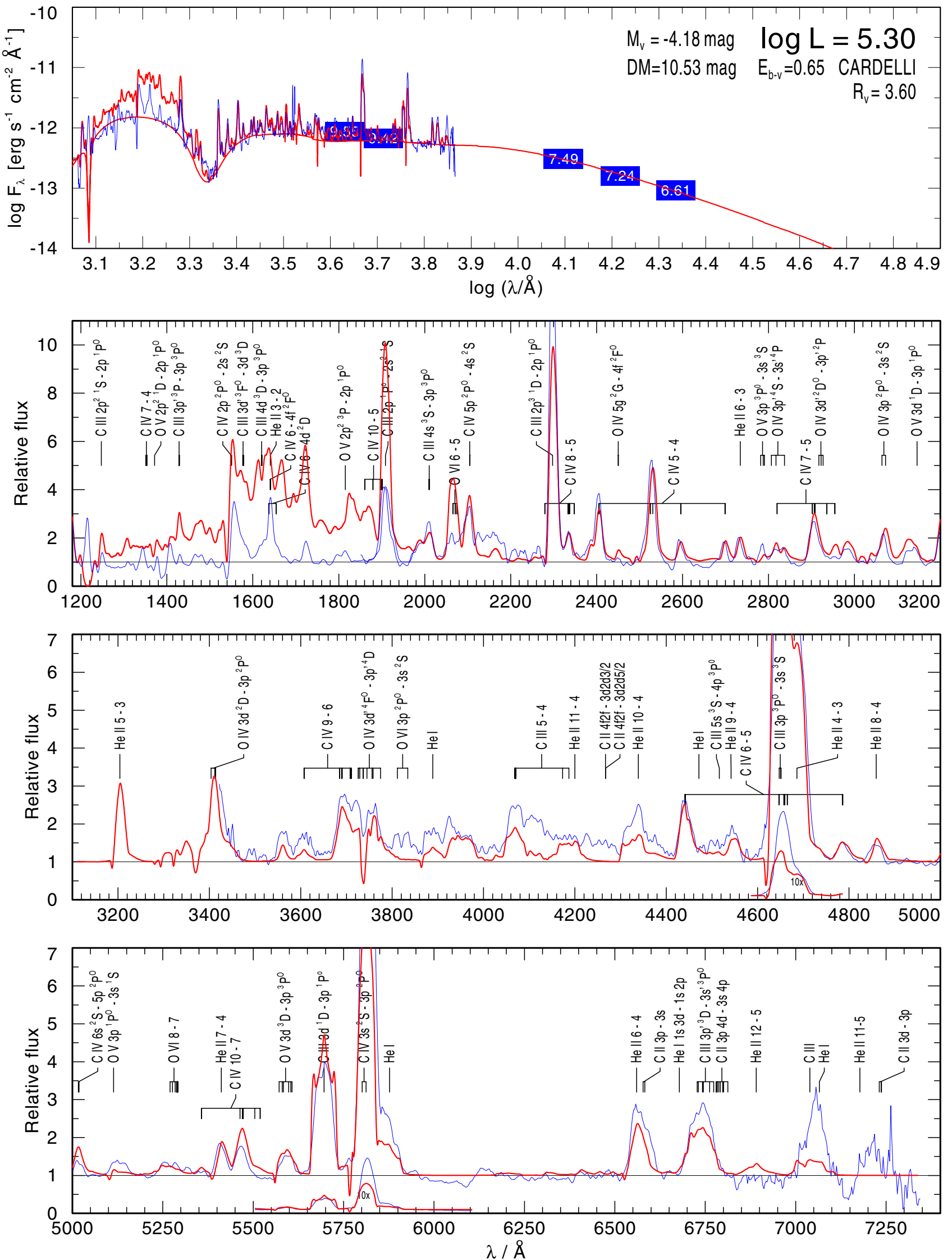

Fig. B.5. Spectral fit for WR 14 . 
A. Sander et al.: The Galactic WC stars

WR015

$\mathrm{T}_{*}=79 \mathrm{kK} \log \mathrm{R}_{\mathrm{t}}=0.5$

WC6

$\mathrm{v}_{\infty}=2000 \mathrm{~km} / \mathrm{s}-\mathrm{He}: \mathrm{C}: \mathrm{O}=55: 40: 5$
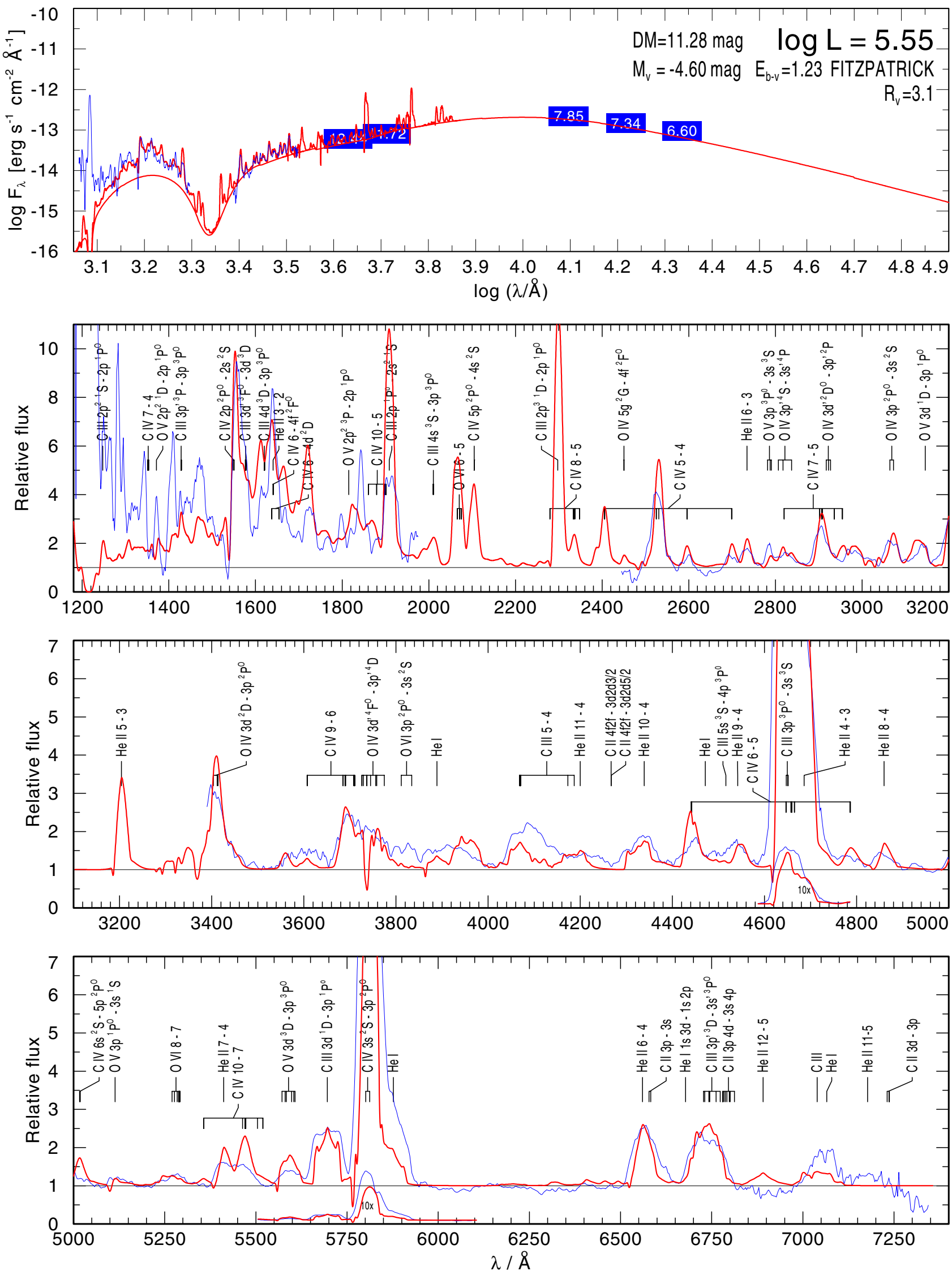

Fig. B.6. Spectral fit for WR 15: the IUE spectrum is underexposed in the range of the interstellar $2200 \AA$ absorption bump. 
WR017

$\mathrm{T}_{*}=79 \mathrm{kK} \log \mathrm{R}_{\mathrm{t}}=0.5$

WC5

$v_{\infty}=2000 \mathrm{~km} / \mathrm{s}-\mathrm{He}: \mathrm{C}: \mathrm{O}=55: 40: 5$
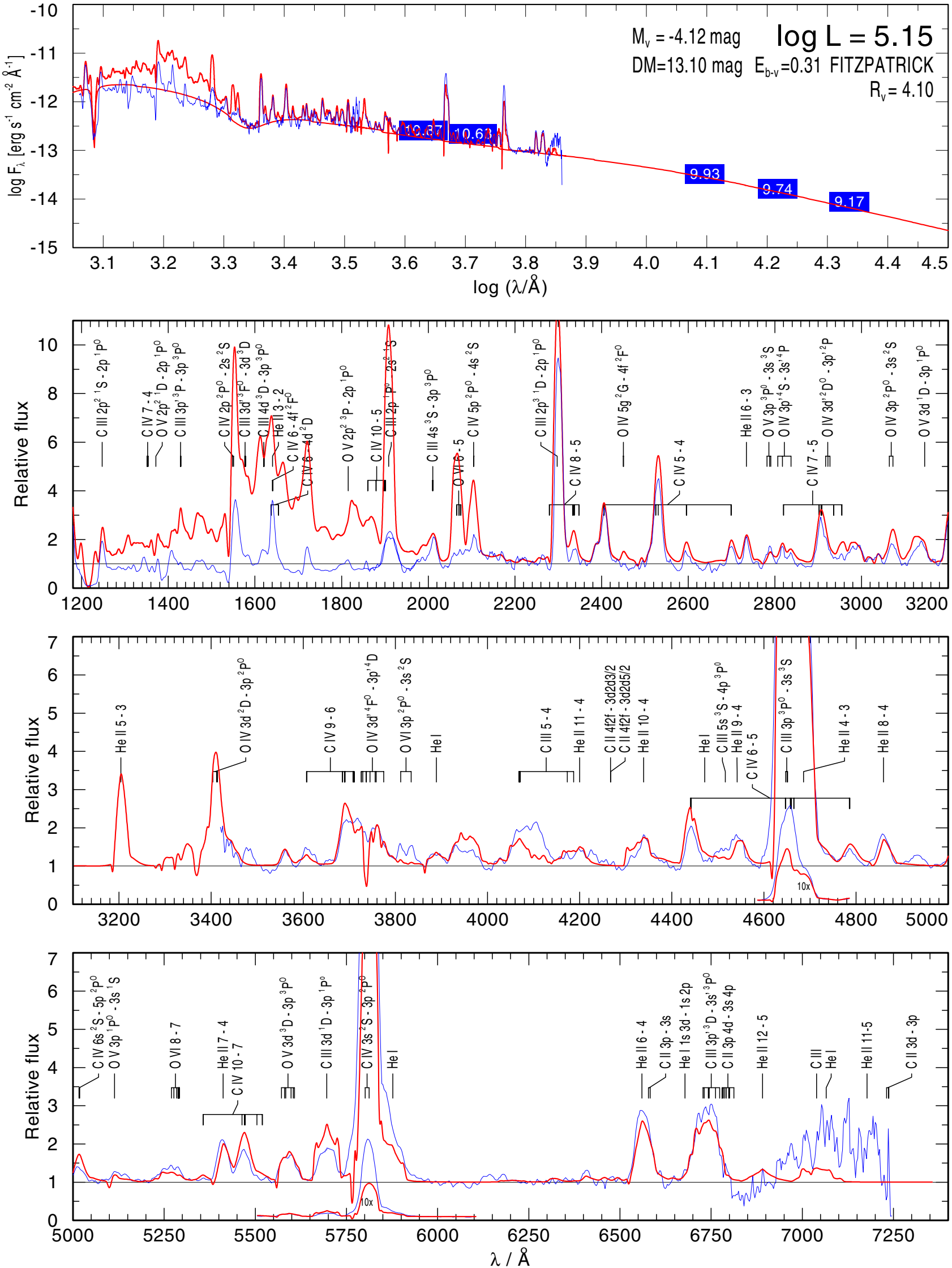

Fig. B.7. Spectral fit for WR 17 . 
WR023

$\mathrm{T}_{\star}=79 \mathrm{kK} \log \mathrm{R}_{\mathrm{t}}=0.5$

WC6

$\mathrm{v}_{\infty}=2000 \mathrm{~km} / \mathrm{s}-\mathrm{He}: \mathrm{C}: \mathrm{O}=55: 40: 5$

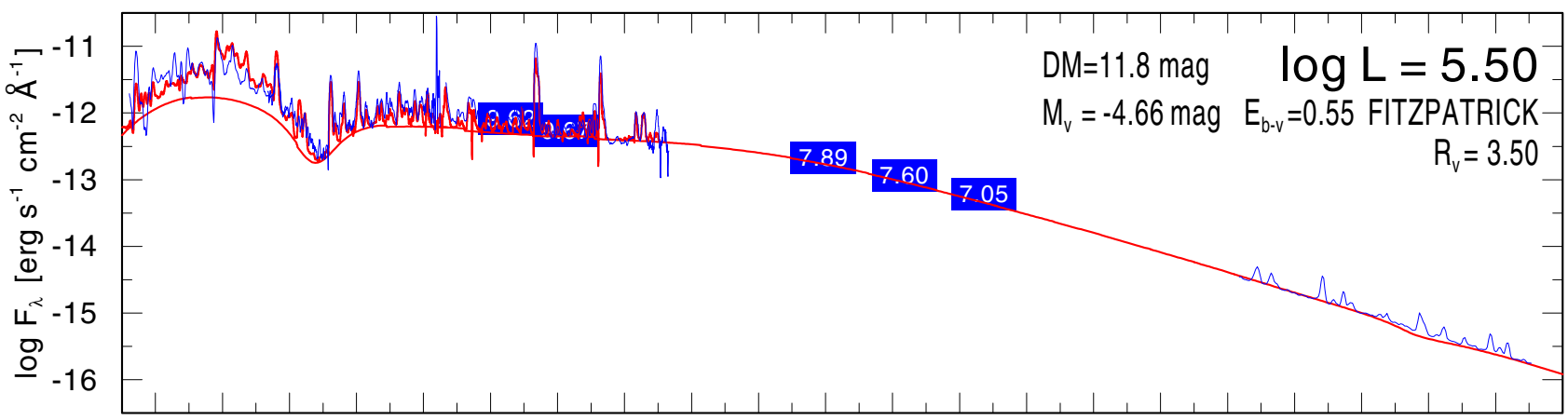

$\begin{array}{llllllllllllllllllllll}3.1 & 3.2 & 3.3 & 3.4 & 3.5 & 3.6 & 3.7 & 3.8 & 3.9 & 4.0 & 4.1 & 4.2 & 4.3 & 4.4 & 4.5 & 4.6 & 4.7 & 4.8 & 4.9 & 5.0 & 5.15 .2\end{array}$ $\log (\lambda / \AA)$
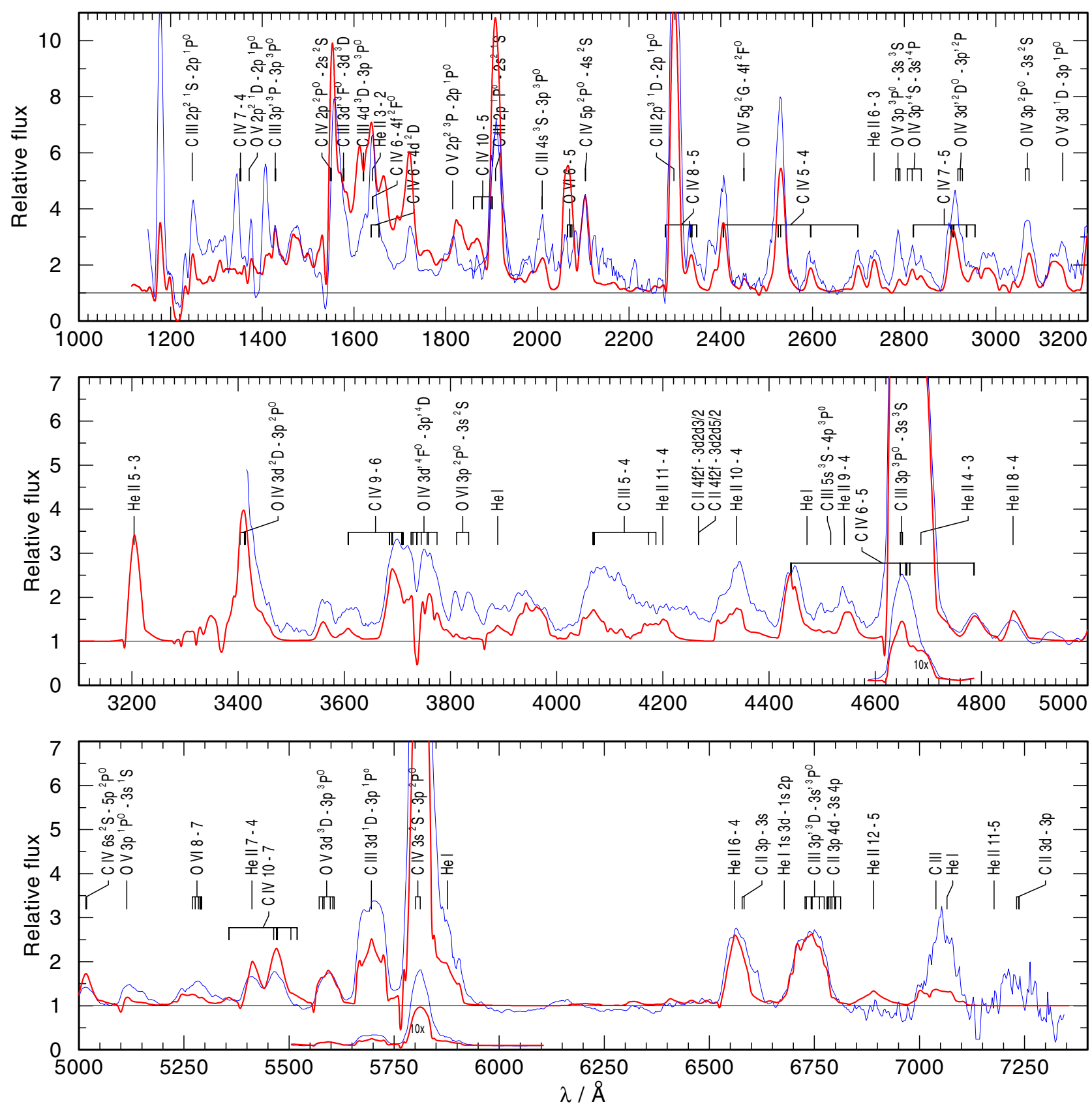

Fig. B.8. Spectral fit for WR 23. 
WR026

WN7/WCE

$T_{*}=79 \mathrm{kK} \log R_{t}=0.6$

$v_{\infty}=1600 \mathrm{~km} / \mathrm{s}-\mathrm{He}: \mathrm{C}: \mathrm{N}=78.5: 20: 1.5$
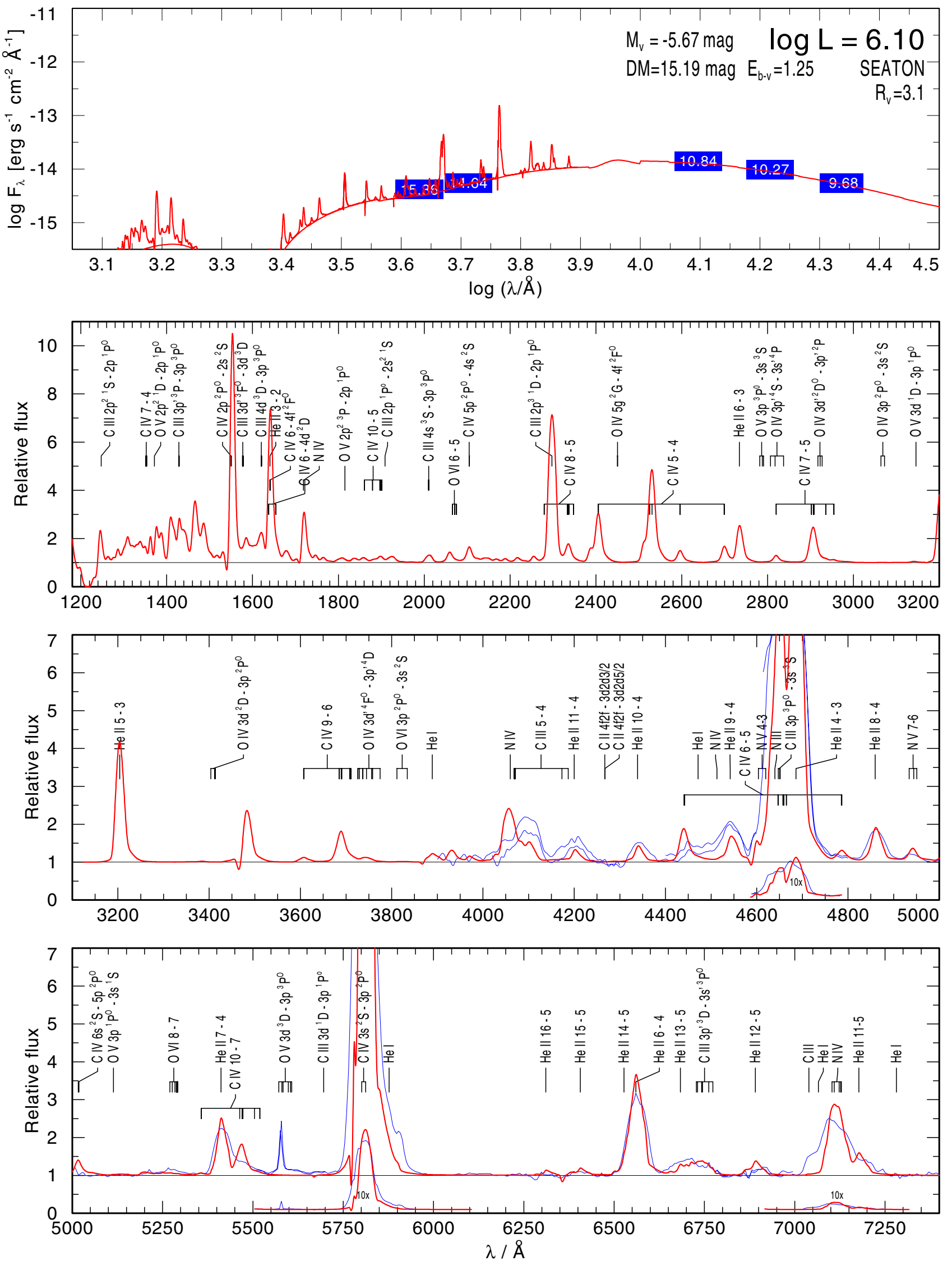

Fig. B.9. Spectral fit for WR 26. 
A. Sander et al.: The Galactic WC stars

WR027

$\mathrm{T}_{*}=79 \mathrm{kK} \log \mathrm{R}_{\mathrm{t}}=0.5$

WC6

$v_{\infty}=2000 \mathrm{~km} / \mathrm{s}-\mathrm{He}: \mathrm{C}: \mathrm{O}=55: 40: 5$
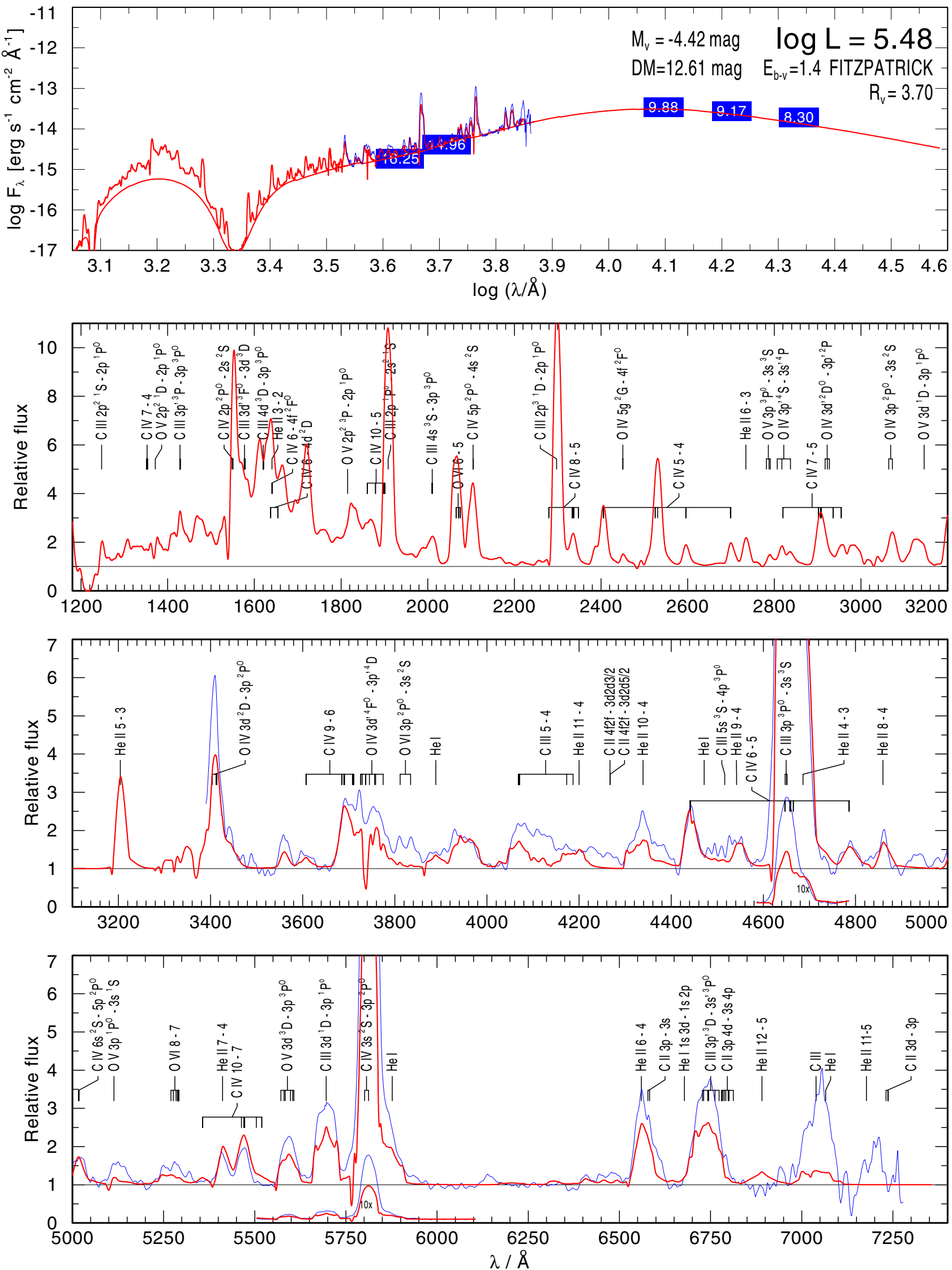

Fig. B.10. Spectral fit for WR 27. 
WR033

$T_{*}=79 \mathrm{kK} \log R_{t}=0.5$

WC5

$\mathrm{v}_{\infty}=2000 \mathrm{~km} / \mathrm{s}-\mathrm{He}: \mathrm{C}: \mathrm{O}=55: 40: 5$
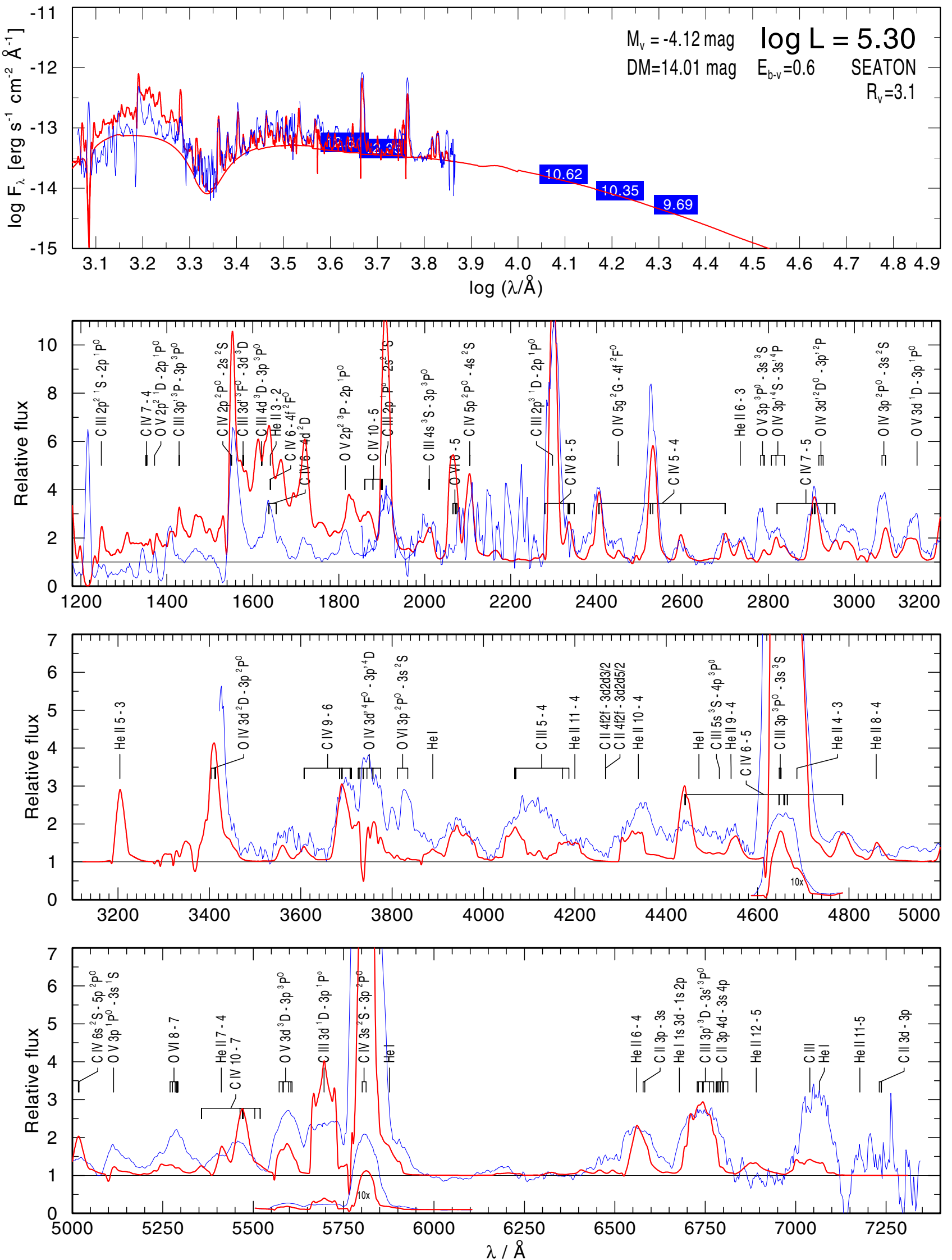

Fig. B.11. Spectral fit for WR 33. 
WR038

$T_{*}=126 \mathrm{kK} \log R_{\mathrm{t}}=0.1$
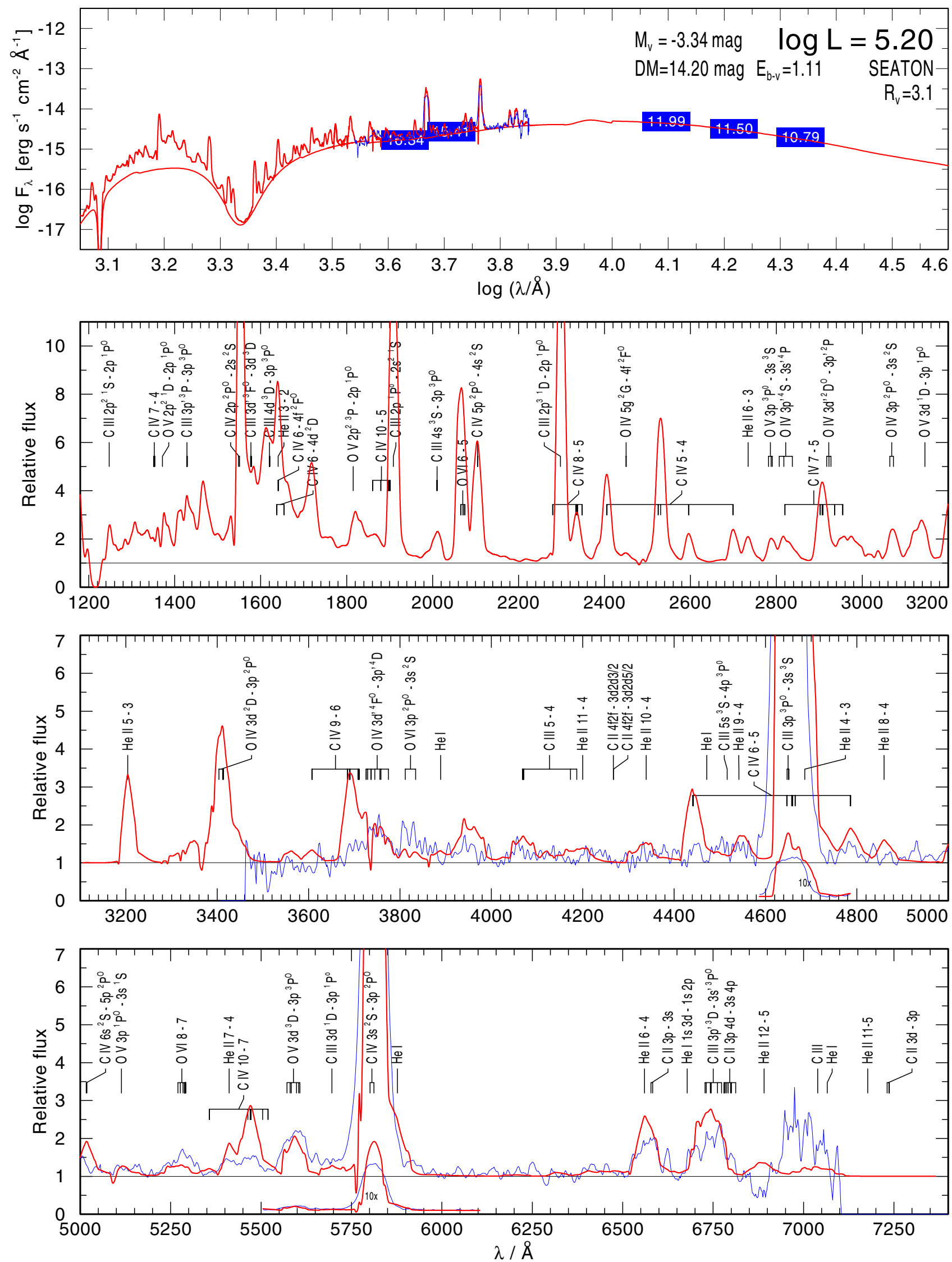

Fig. B.12. Spectral fit for WR 38 . 
WR039 (Binary)

$\mathrm{T}_{*}=56 \mathrm{kK} \log \mathrm{R}_{\mathrm{t}}=1.1$ $v_{\infty}=2000 \mathrm{~km} / \mathrm{s}-\mathrm{He}: \mathrm{C}: \mathrm{O}=55: 40: 5$
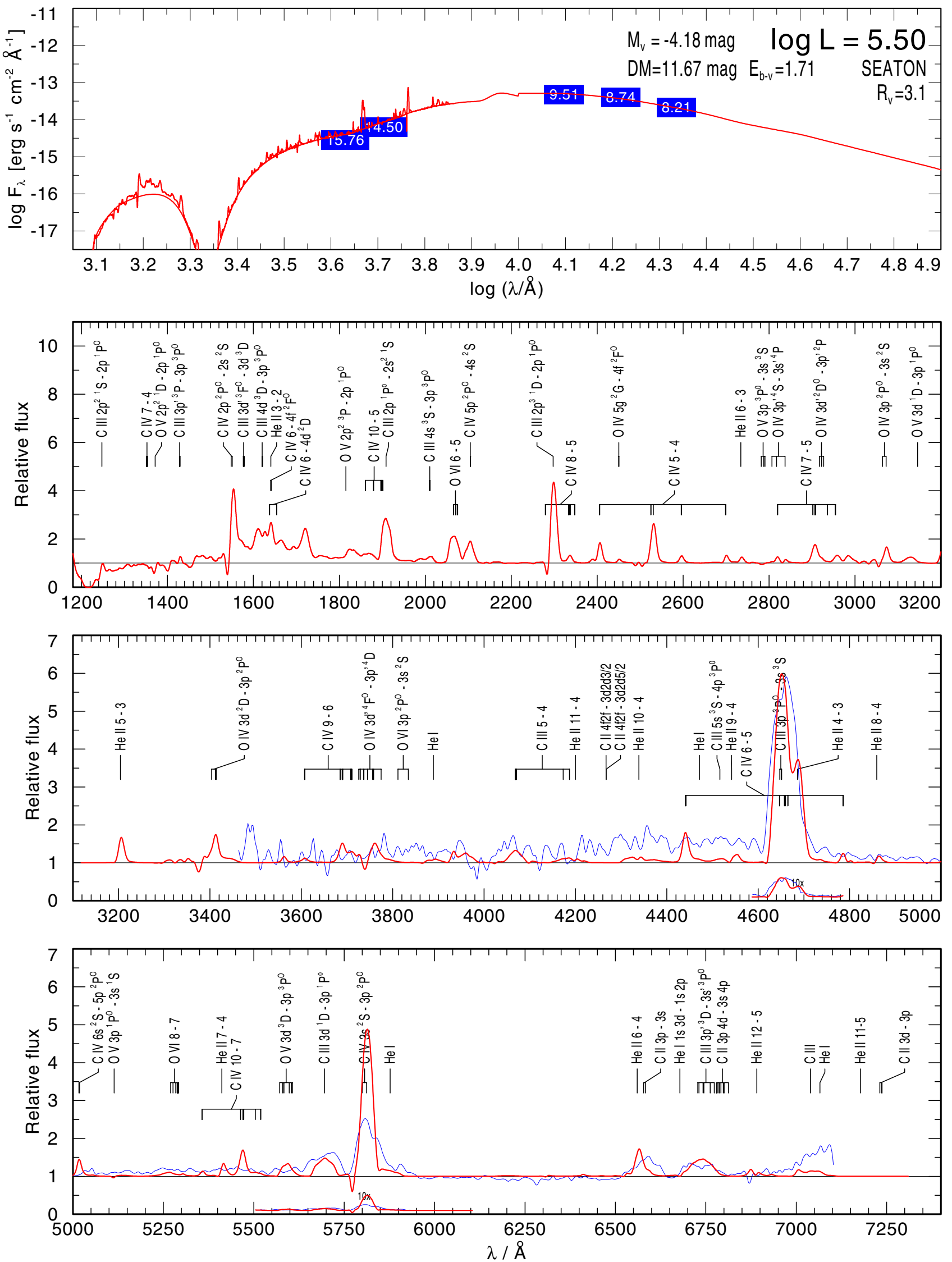

Fig. B.13. Spectral pseudo fit for WR 39. 
A. Sander et al.: The Galactic WC stars

WR045

$\mathrm{T}_{*}=79 \mathrm{kK} \log \mathrm{R}_{\mathrm{t}}=0.5$

WC6

$v_{\infty}=2000 \mathrm{~km} / \mathrm{s}-\mathrm{He}: \mathrm{C}: \mathrm{O}=55: 40: 5$
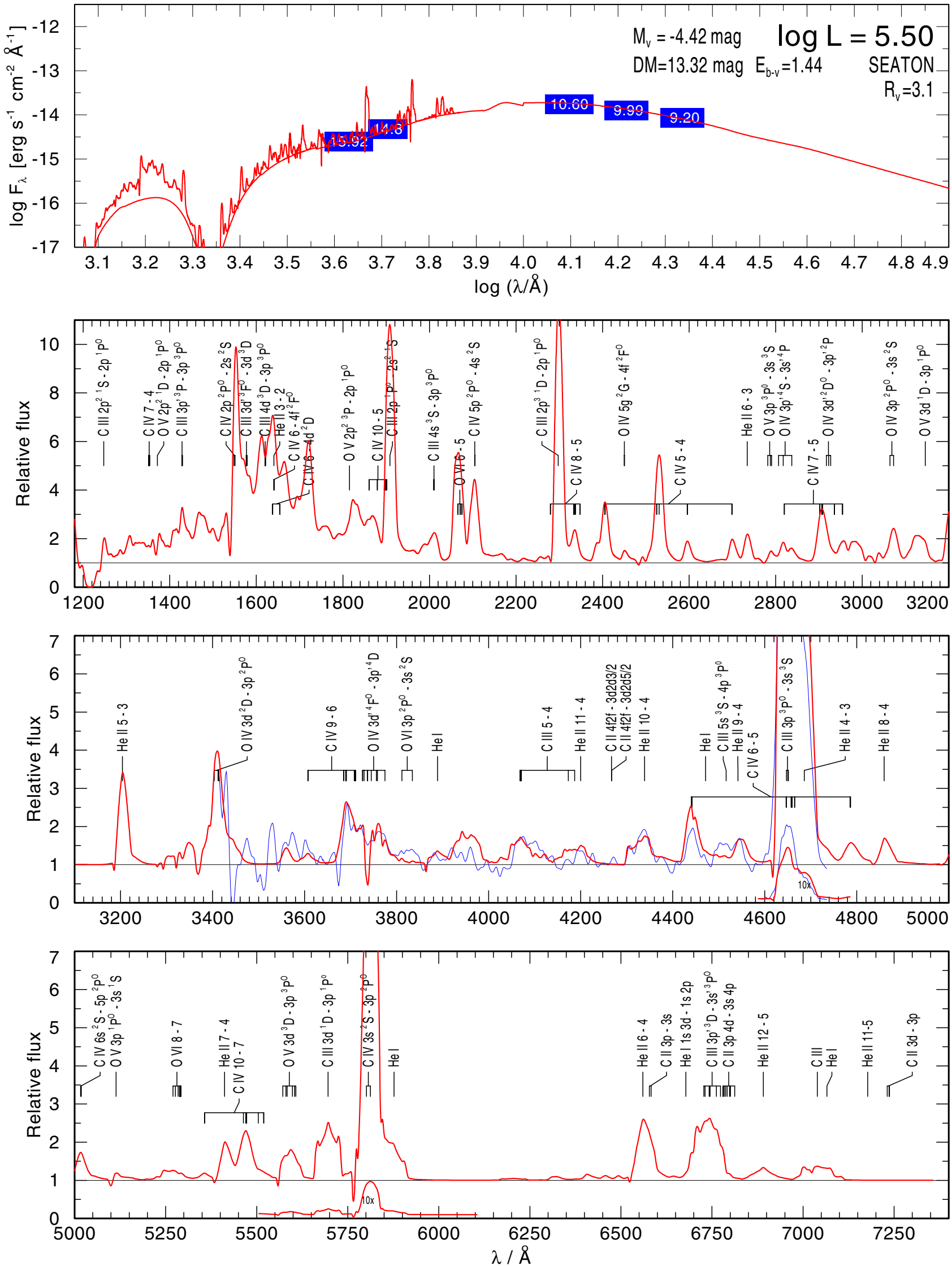

Fig. B.14. Spectral fit for WR 45. 
WR050 (Binary)

WC7

$\mathrm{T}_{*}=56 \mathrm{kK} \log \mathrm{R}_{\mathrm{t}}=1.0$

$v_{\infty}=2000 \mathrm{~km} / \mathrm{s}-\mathrm{He}: \mathrm{C}: \mathrm{O}=55: 40: 5$
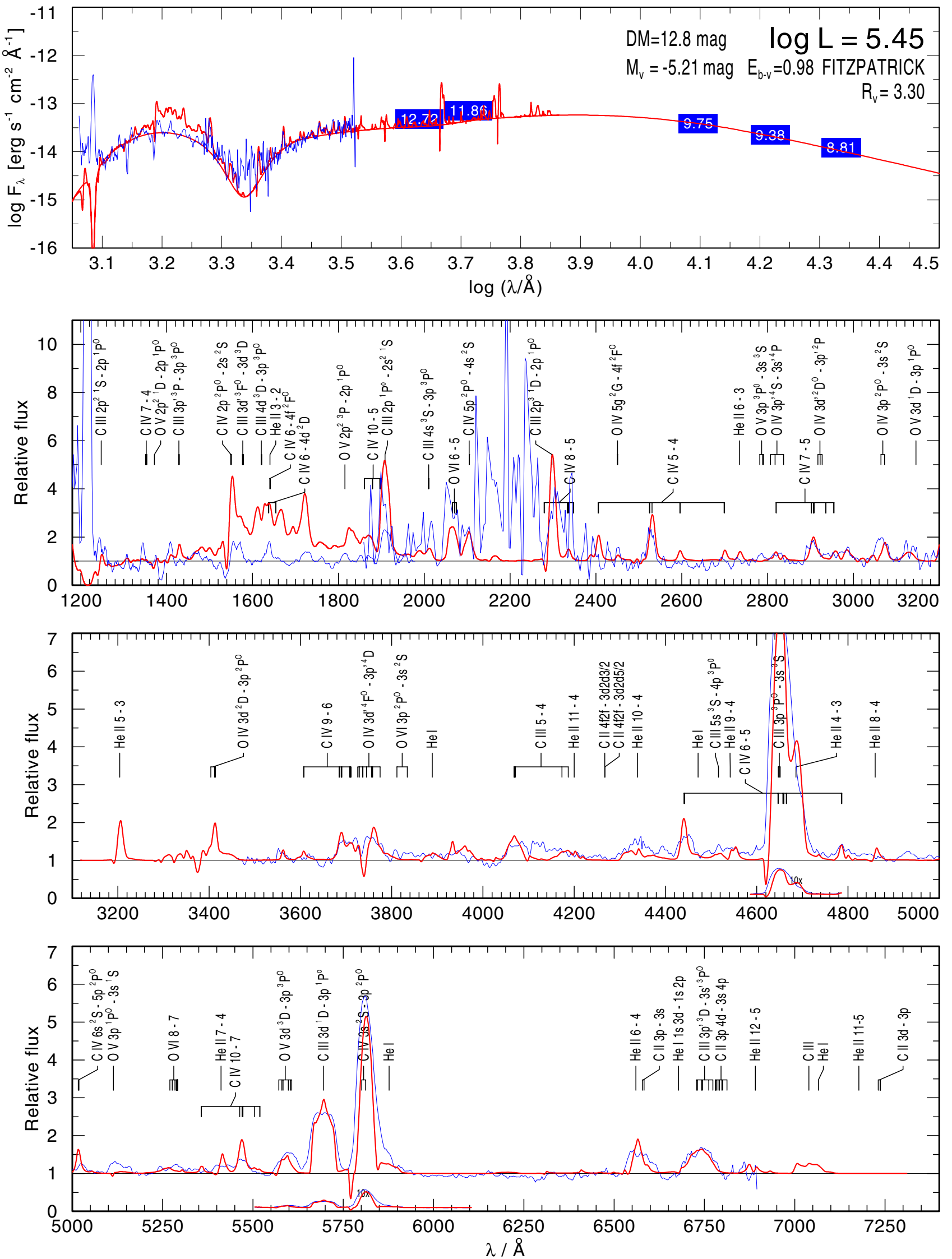

Fig. B.15. Spectral pseudo fit for WR 50. 

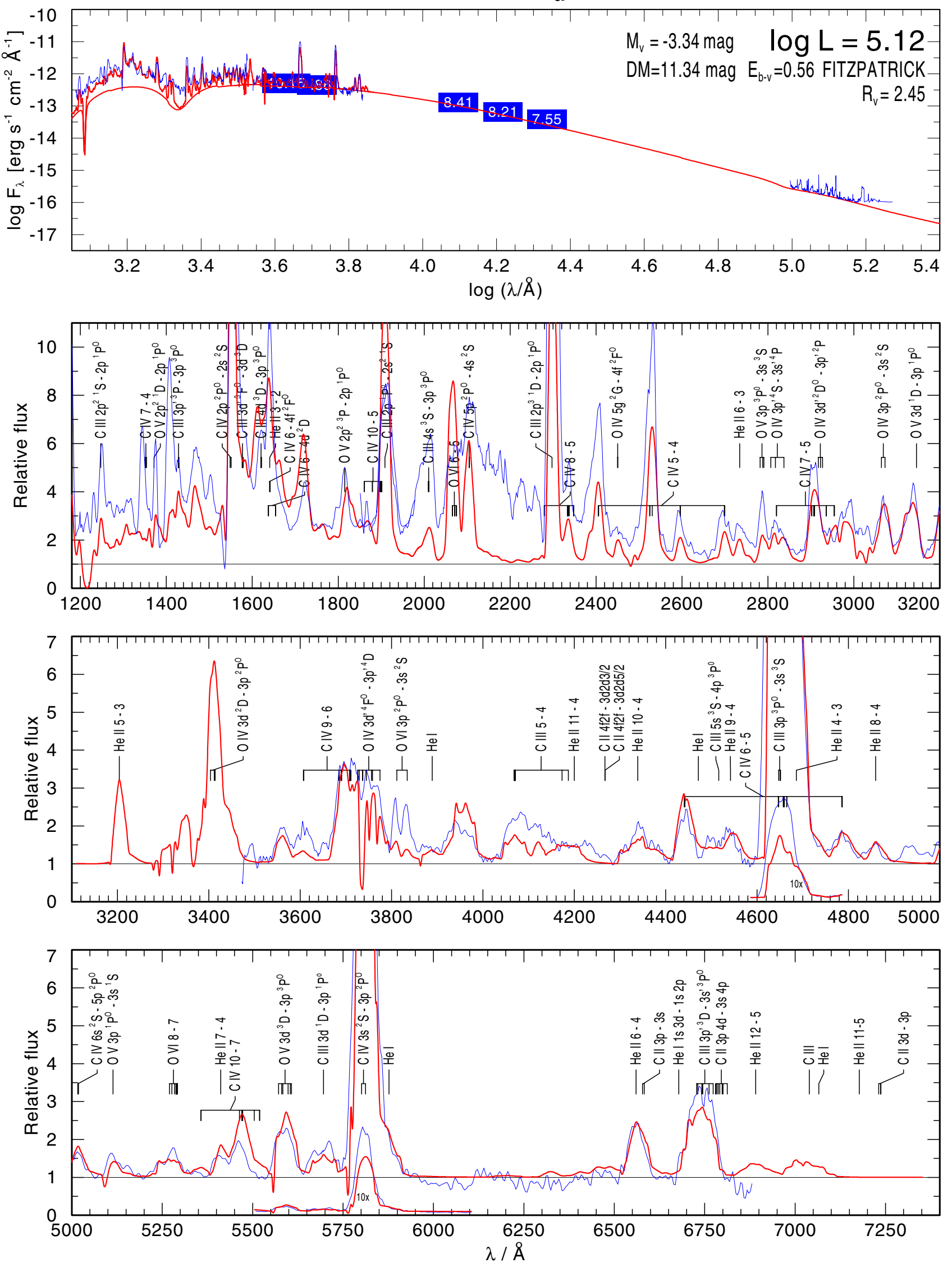

Fig. B.16. Spectral fit for WR 52. Note that the model is calculated with an oxygen mass fraction of $15 \%$. 
WR053

WC8d

$\mathrm{T}_{*}=50 \mathrm{kK} \log \mathrm{R}_{\mathrm{t}}=0.9$

$v_{\infty}=2000 \mathrm{~km} / \mathrm{s}-\mathrm{He}: \mathrm{C}: \mathrm{O}=55: 40: 5$
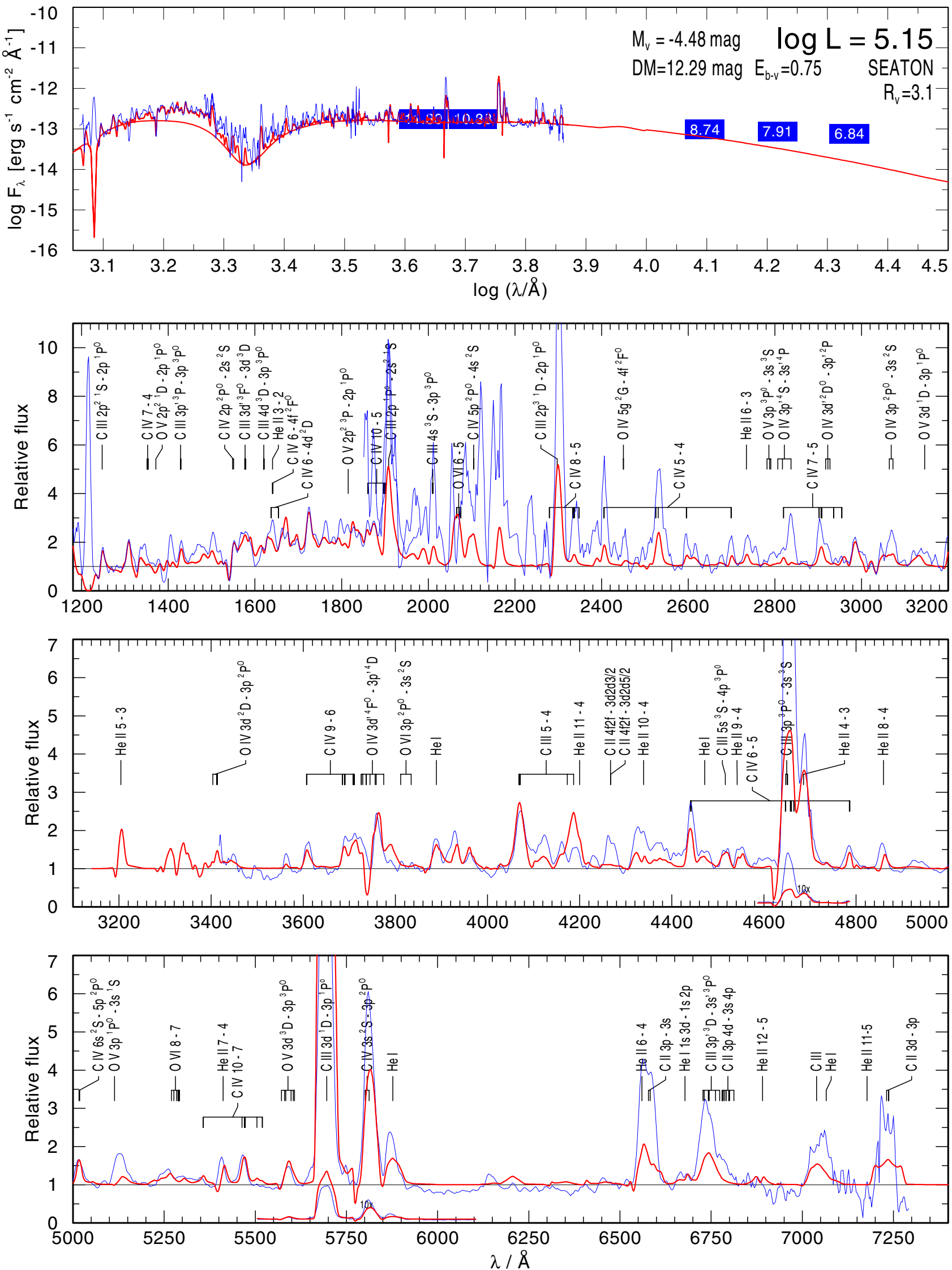

Fig. B.17. Spectral fit for WR 53. 
WR056
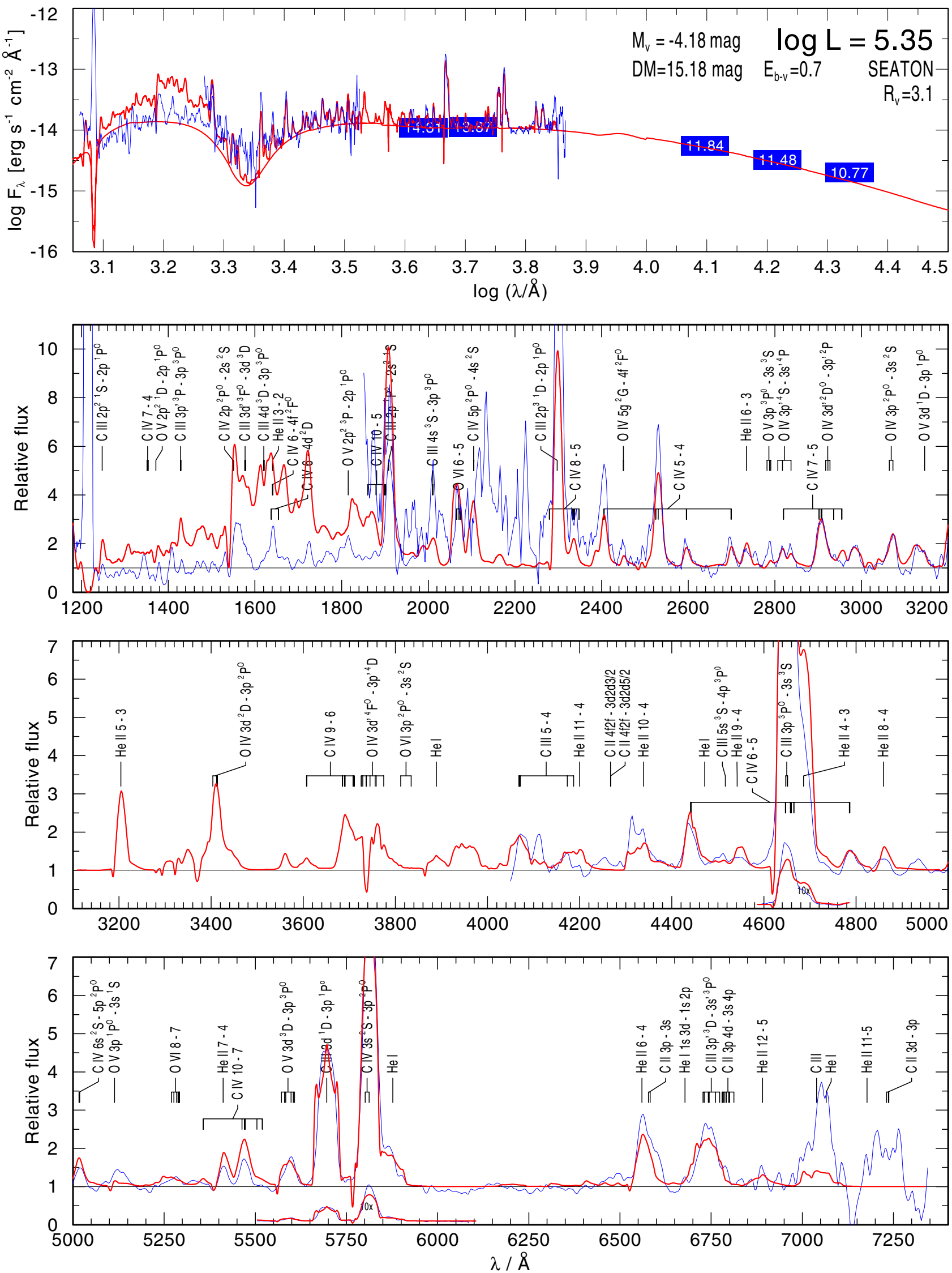

Fig. B.18. Spectral fit for WR 56. 
WR057

WC8

$T_{*}=63 \mathrm{kK} \log R_{t}=0.7$

$v_{\infty}=2000 \mathrm{~km} / \mathrm{s}-\mathrm{He}: \mathrm{C}: \mathrm{O}=55: 40: 5$
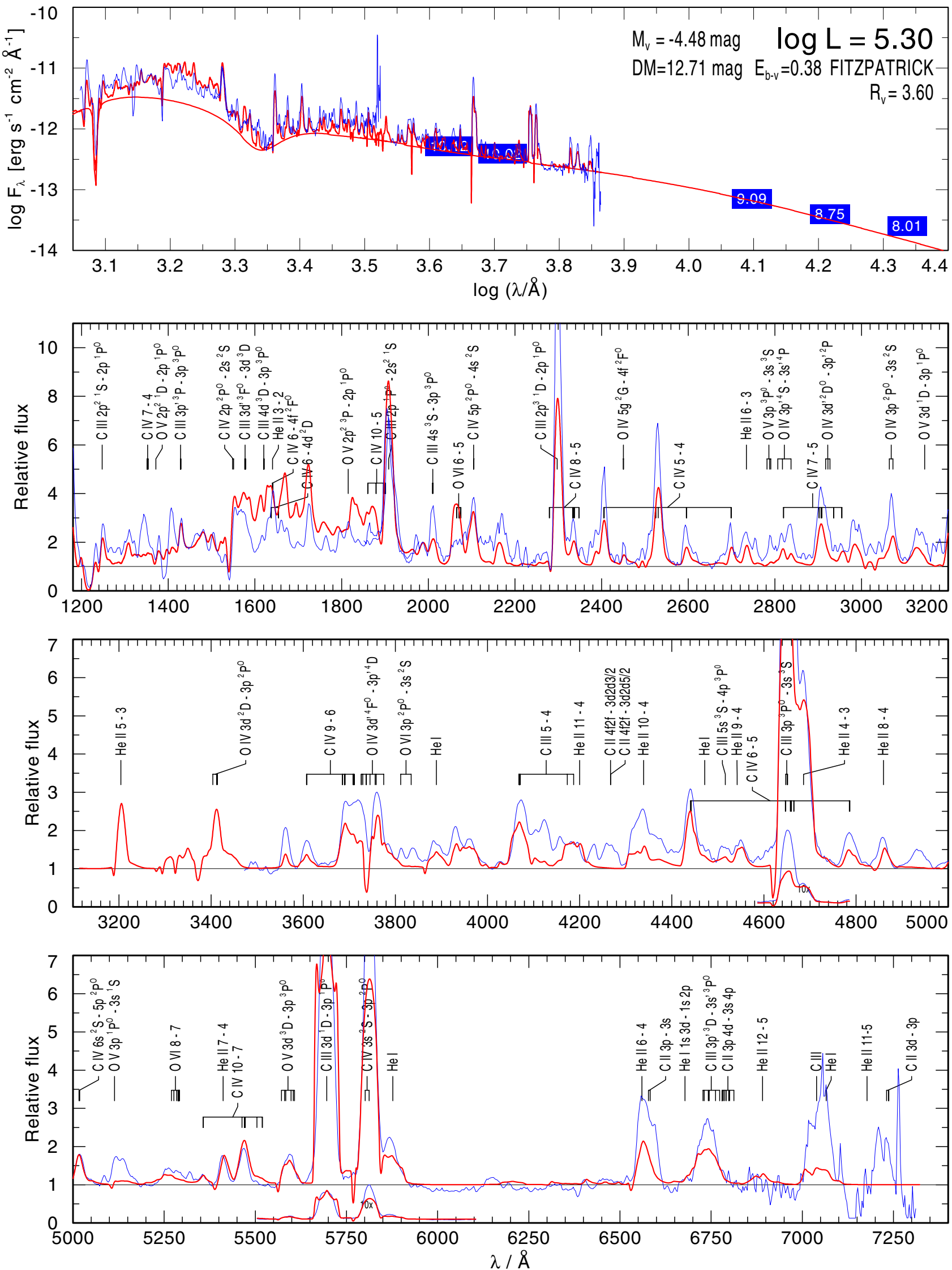

Fig. B.19. Spectral fit for WR 57. 
WR058

WN4/WCE

$\mathrm{T}_{*}=79 \mathrm{kK} \log \mathrm{R}_{\mathrm{t}}=0.5$

$v_{\infty}=1600 \mathrm{~km} / \mathrm{s}-\mathrm{He}: \mathrm{C}: \mathrm{N}=98: 0.1: 1.5$
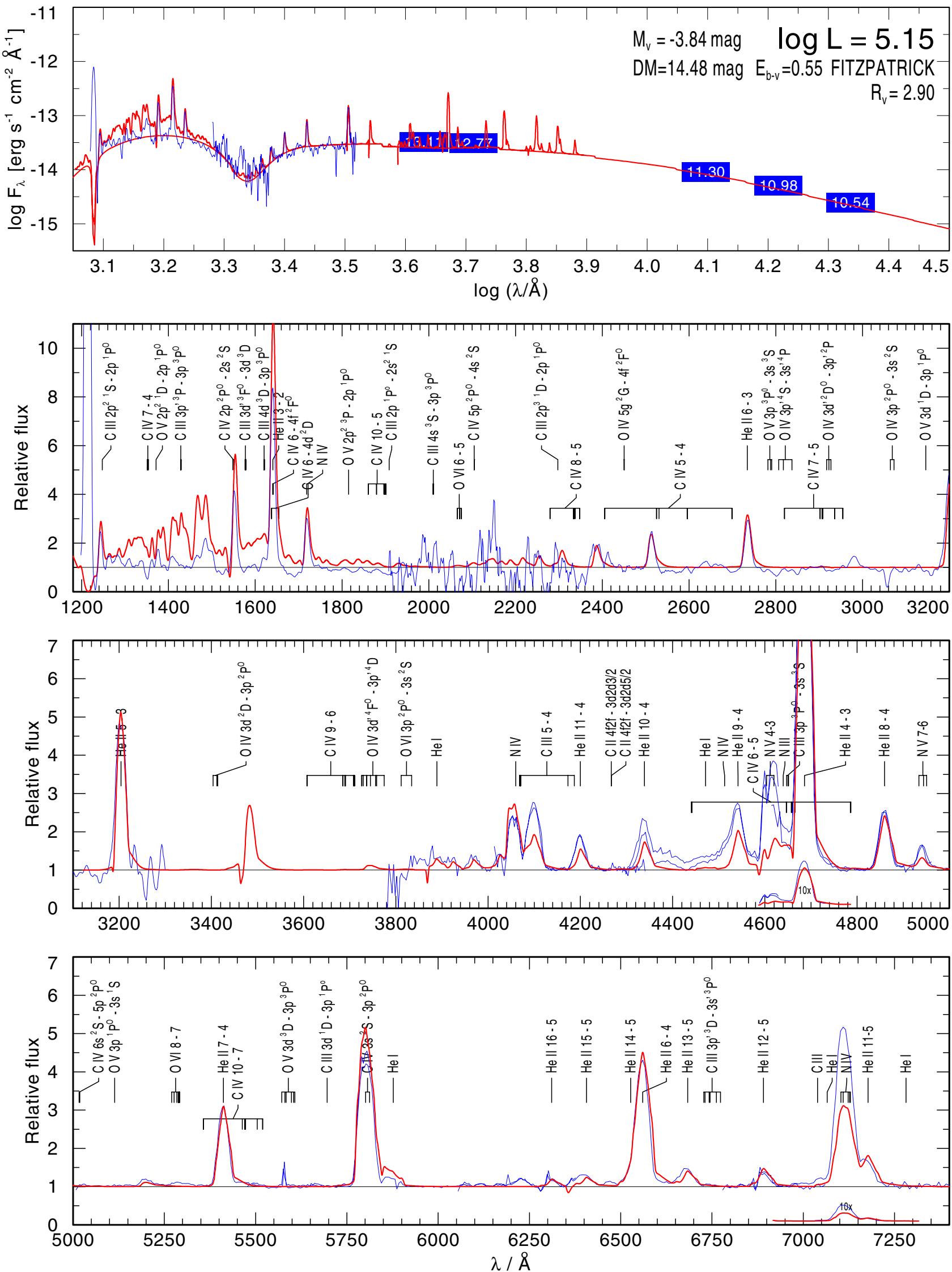

Fig. B.20. Spectral fit for WR 58 . 
WR059

WC9d

$\mathrm{T}_{*}=40 \mathrm{kK} \log \mathrm{R}_{\mathrm{t}}=1.0$

$v_{\infty}=1600 \mathrm{~km} / \mathrm{s}-\mathrm{He}: \mathrm{C}: \mathrm{O}=55: 40: 5$
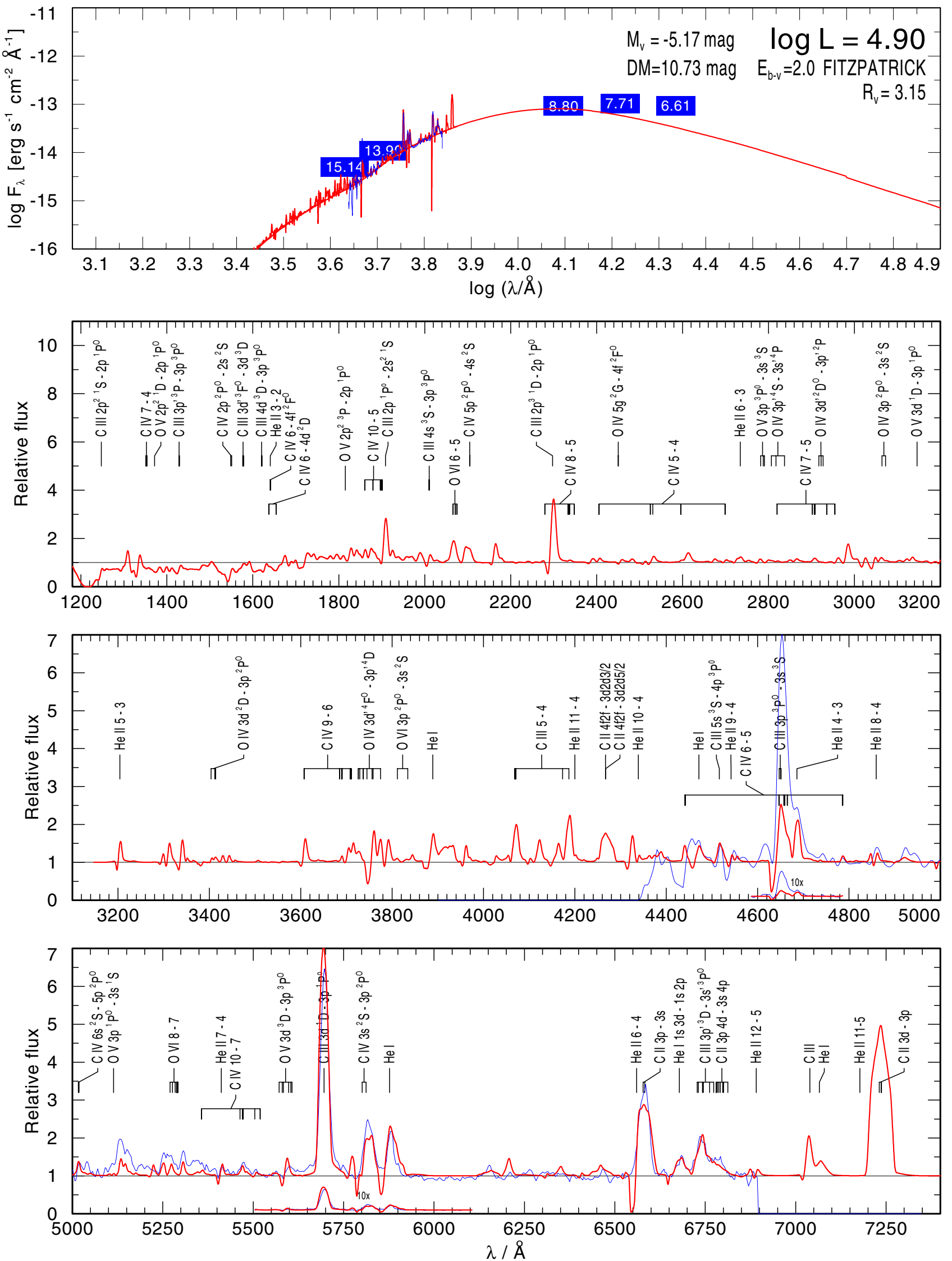

Fig. B.21. Spectral fit for WR 59. 

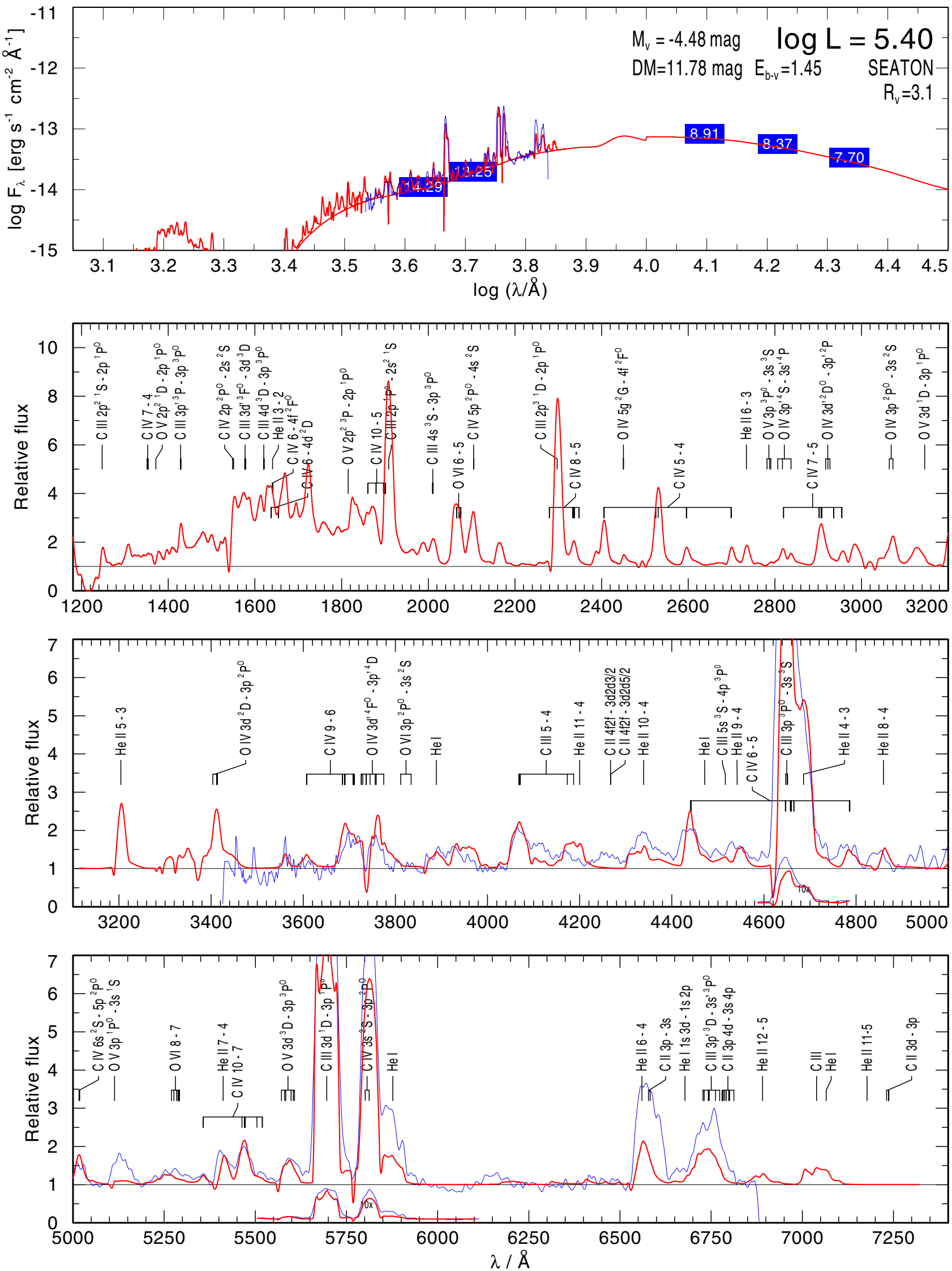

Fig. B.22. Spectral fit for WR 60 . 
WR064
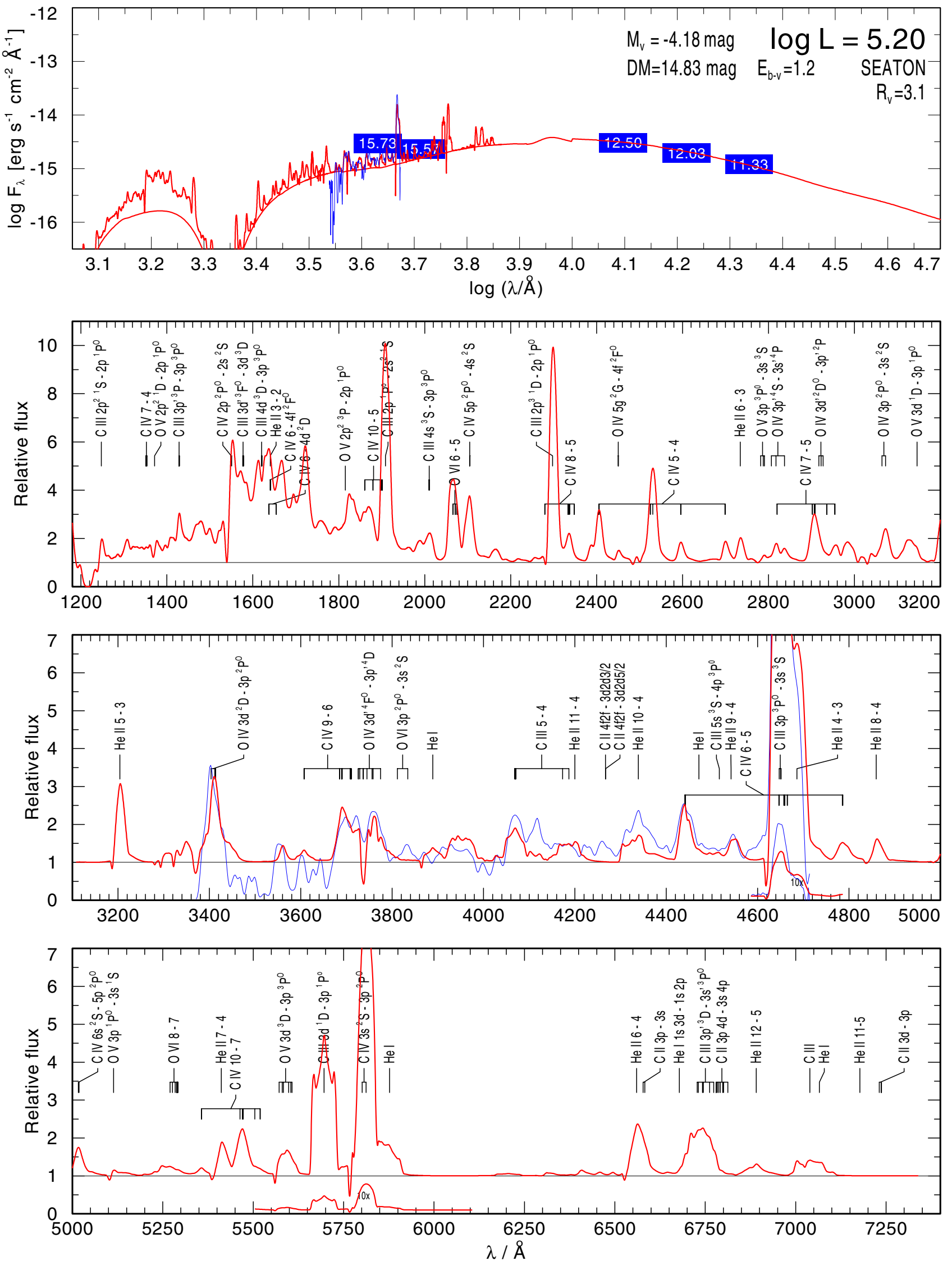

Fig. B.23. Spectral fit for WR 64 . 

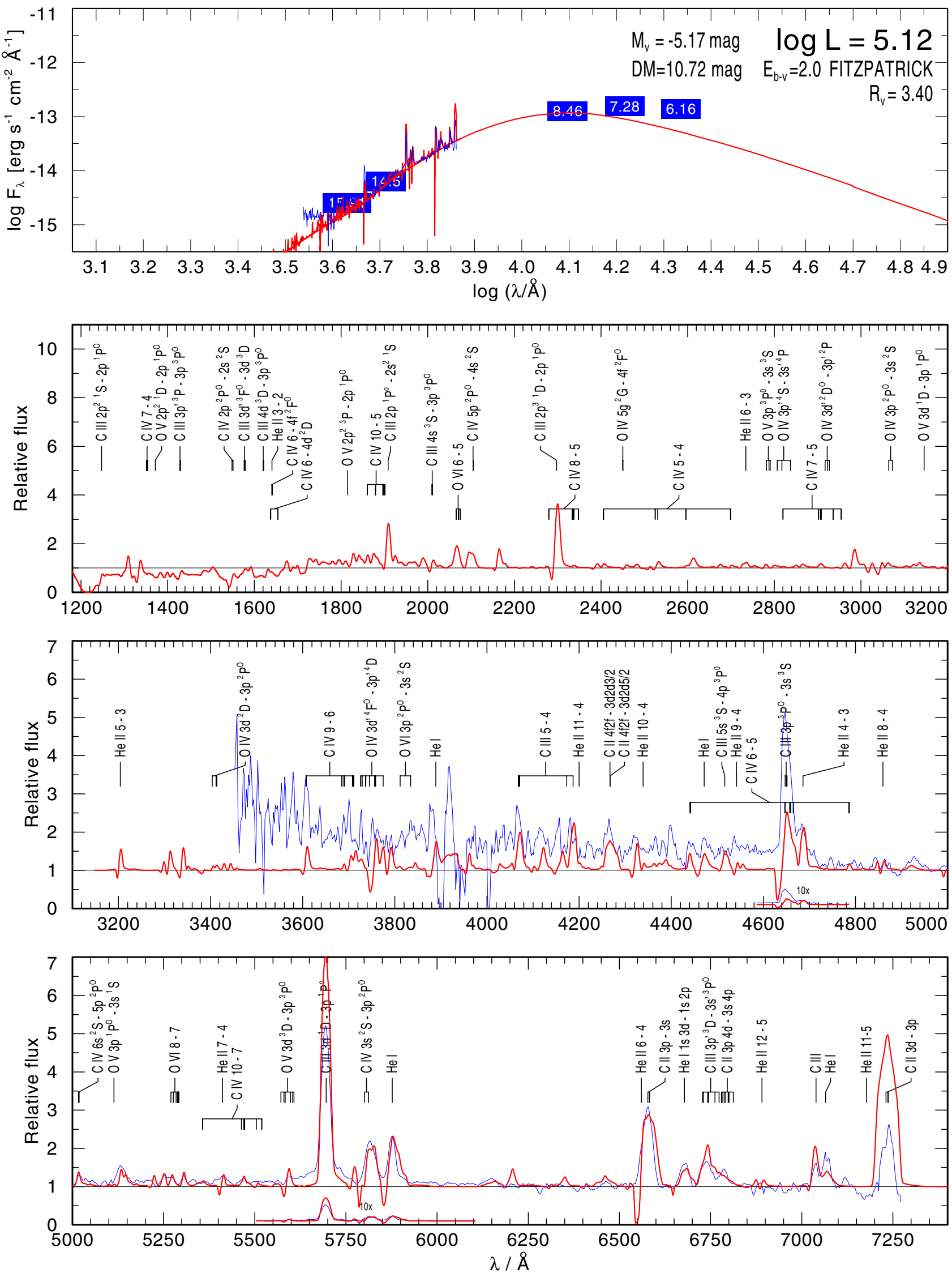

Fig. B.24. Spectral fit for WR 65 . 
WR068

$\mathrm{T}_{*}=71 \mathrm{kK} \log \mathrm{R}_{\mathrm{t}}=0.6$ $\mathrm{v}_{\infty}=2000 \mathrm{~km} / \mathrm{s}-\mathrm{He}: \mathrm{C}: \mathrm{O}=55: 40: 5$
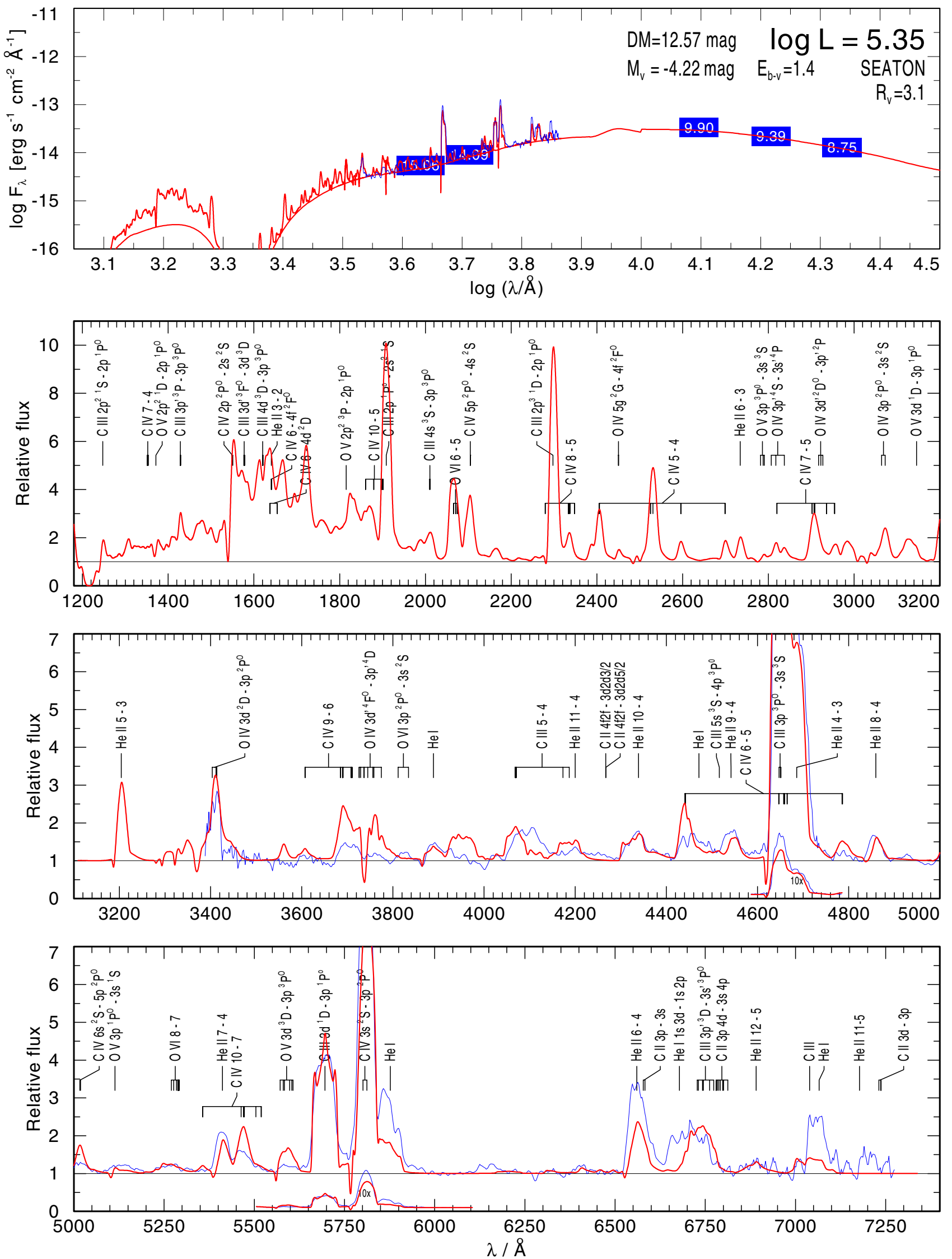

Fig. B.25. Spectral fit for WR 68 . 
WR069

$\mathrm{T}_{*}=40 \mathrm{kK} \log \mathrm{R}_{\mathrm{t}}=1.0$
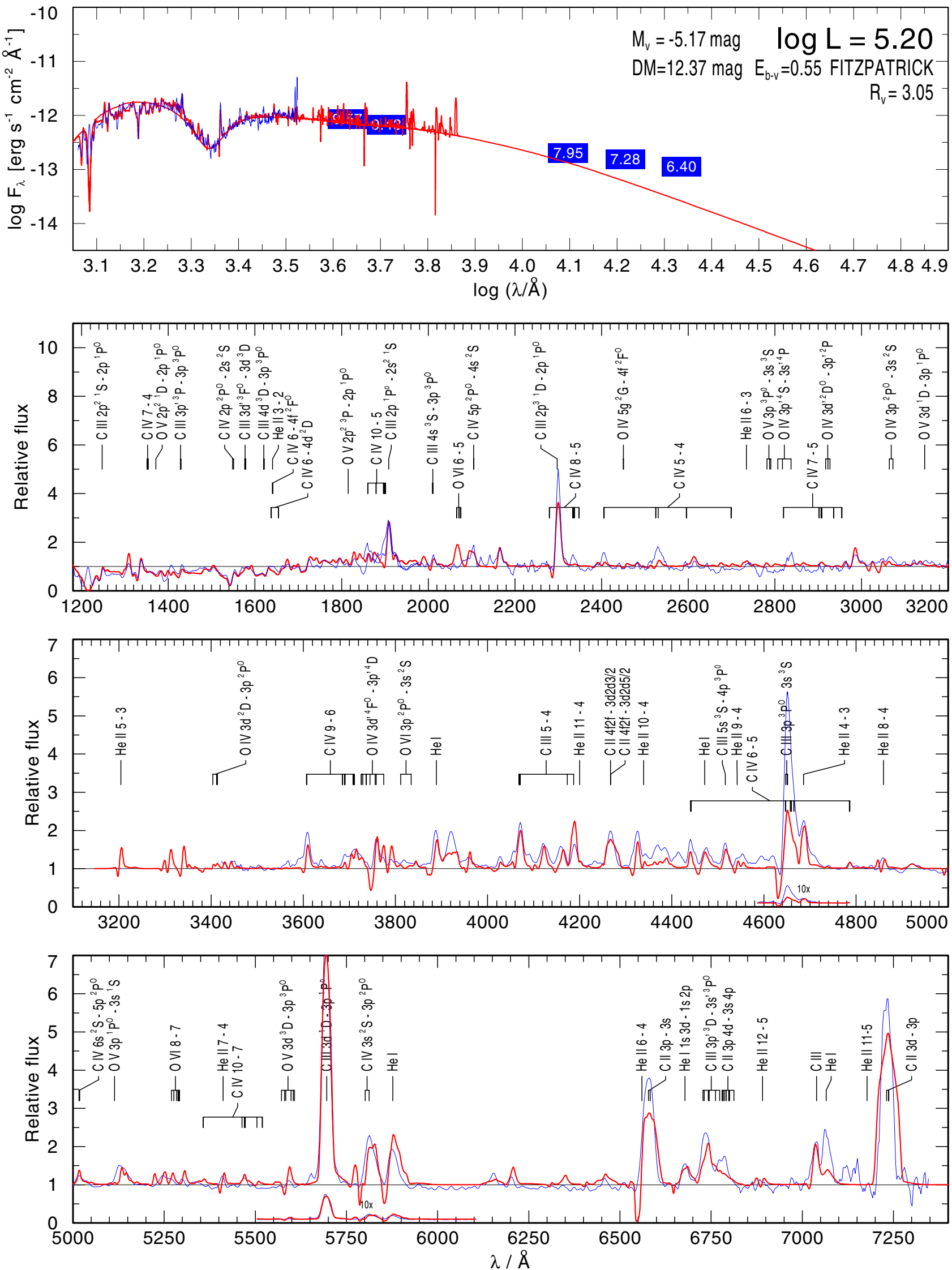

Fig. B.26. Spectral fit for WR 69. 

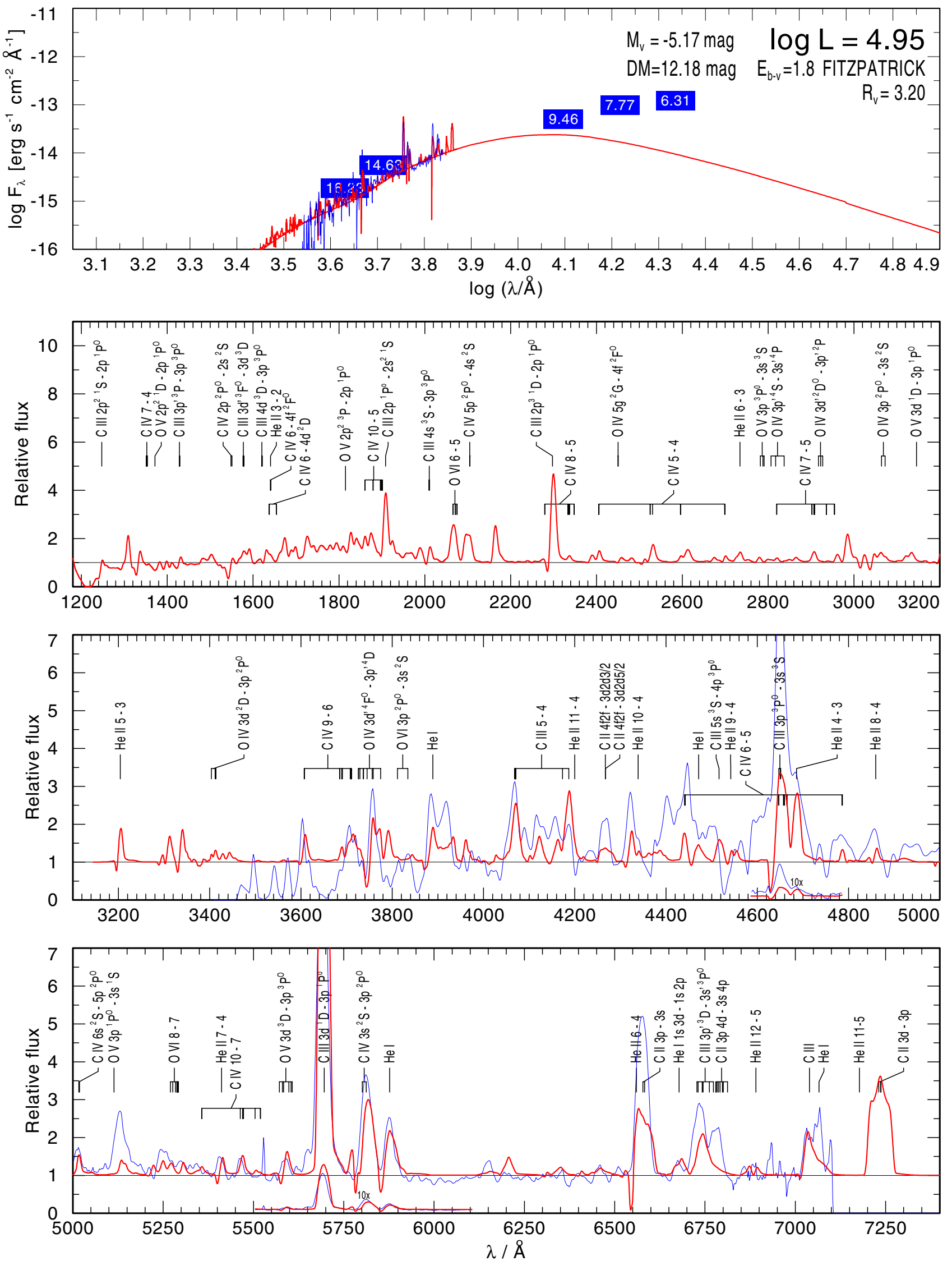

Fig. B.27. Spectral fit for WR 80 . 
WR081
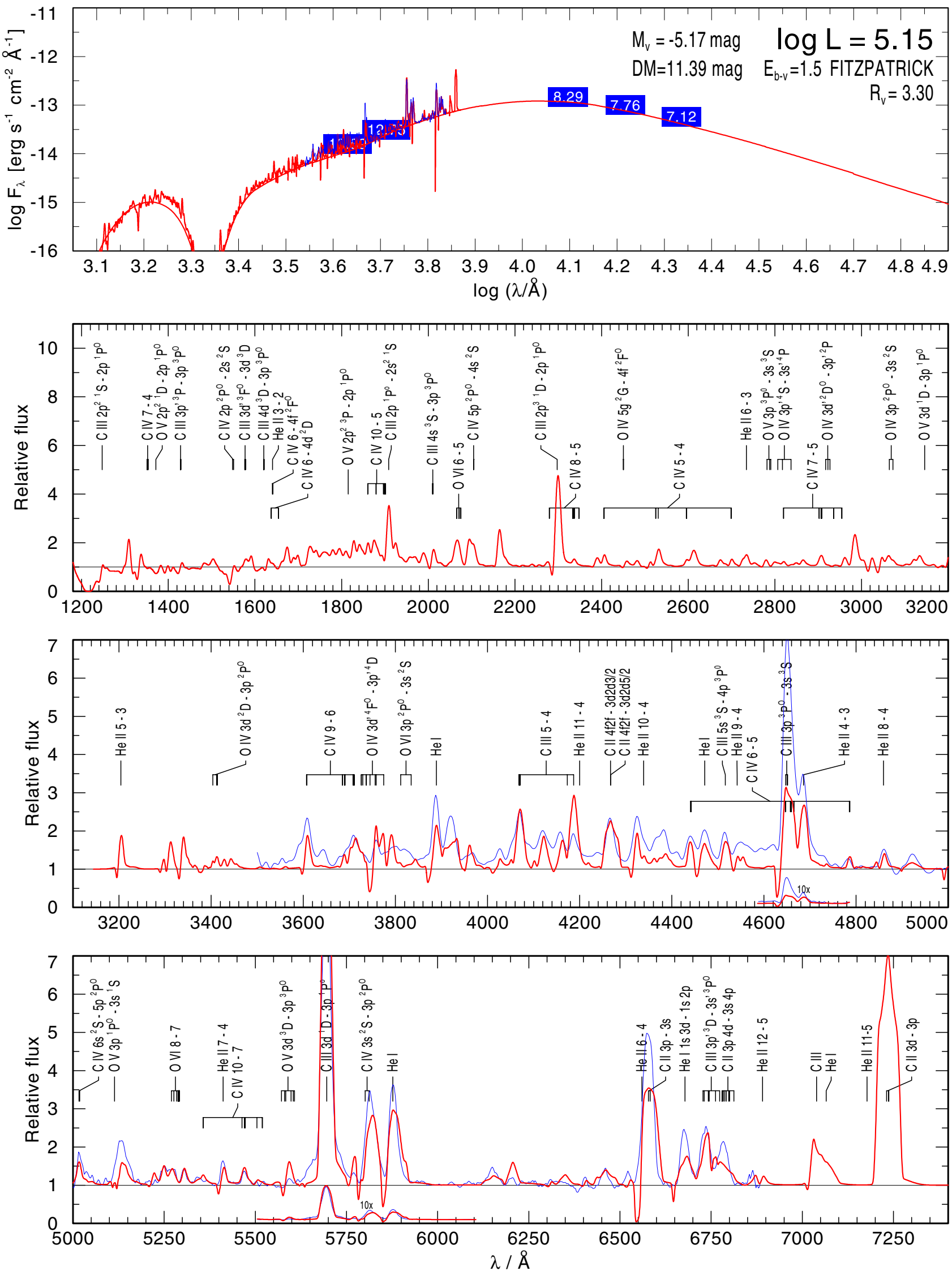

Fig. B.28. Spectral fit for WR 81 . 
WR086 (Binary)
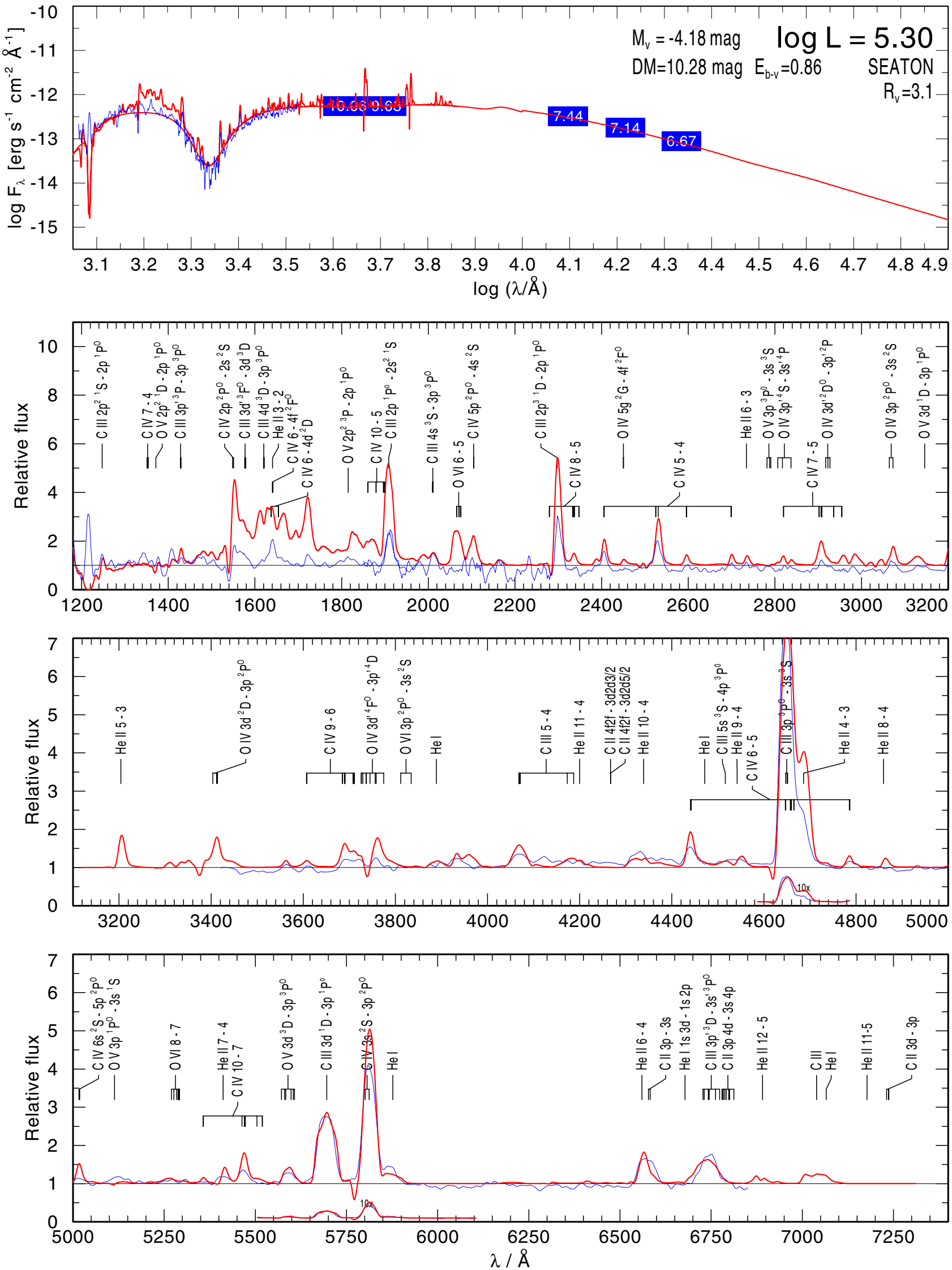

Fig. B.29. Spectral pseudo fit for WR 86 . 
WR088
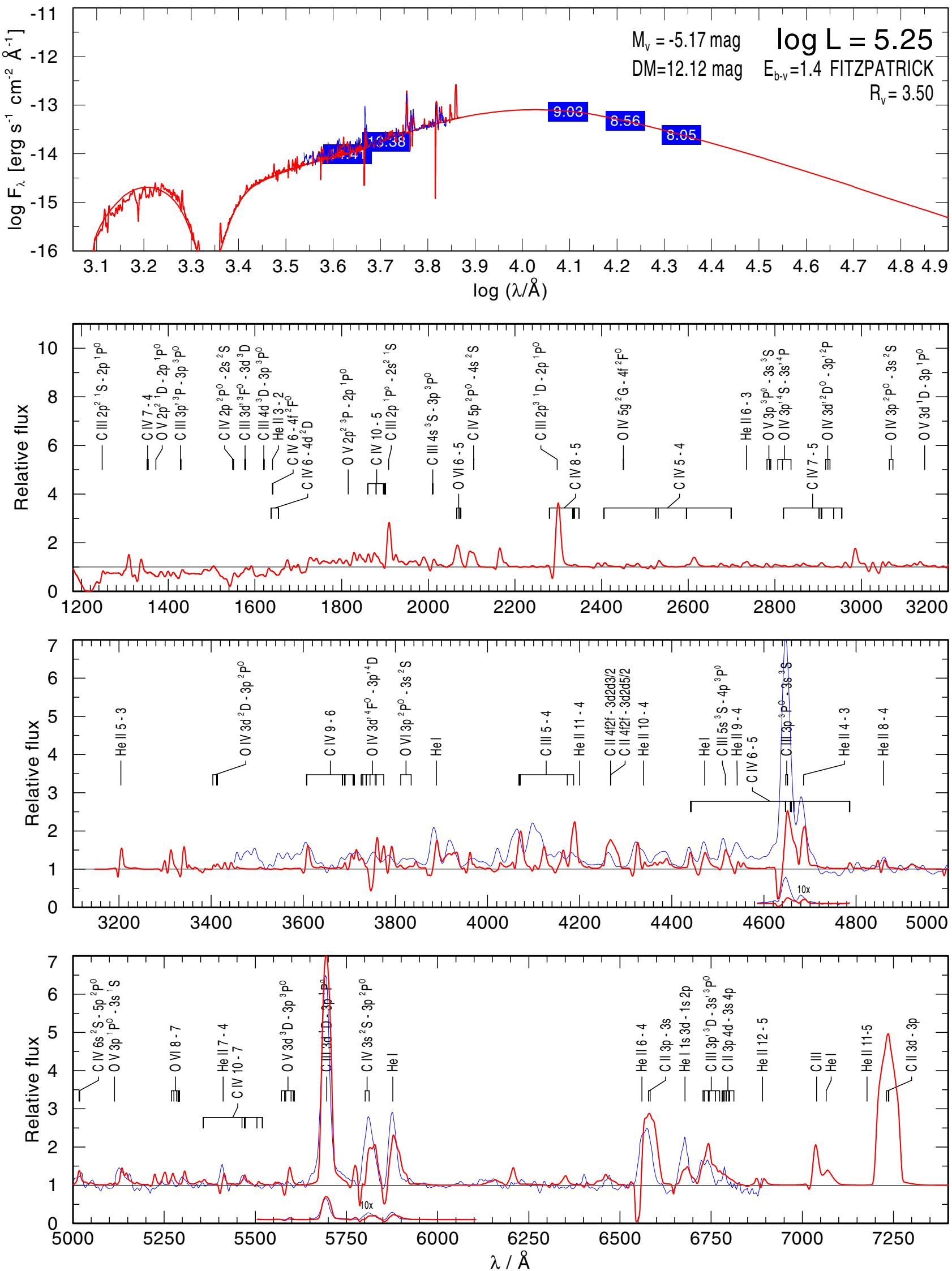

Fig. B.30. Spectral fit for WR 88 . 
WR090
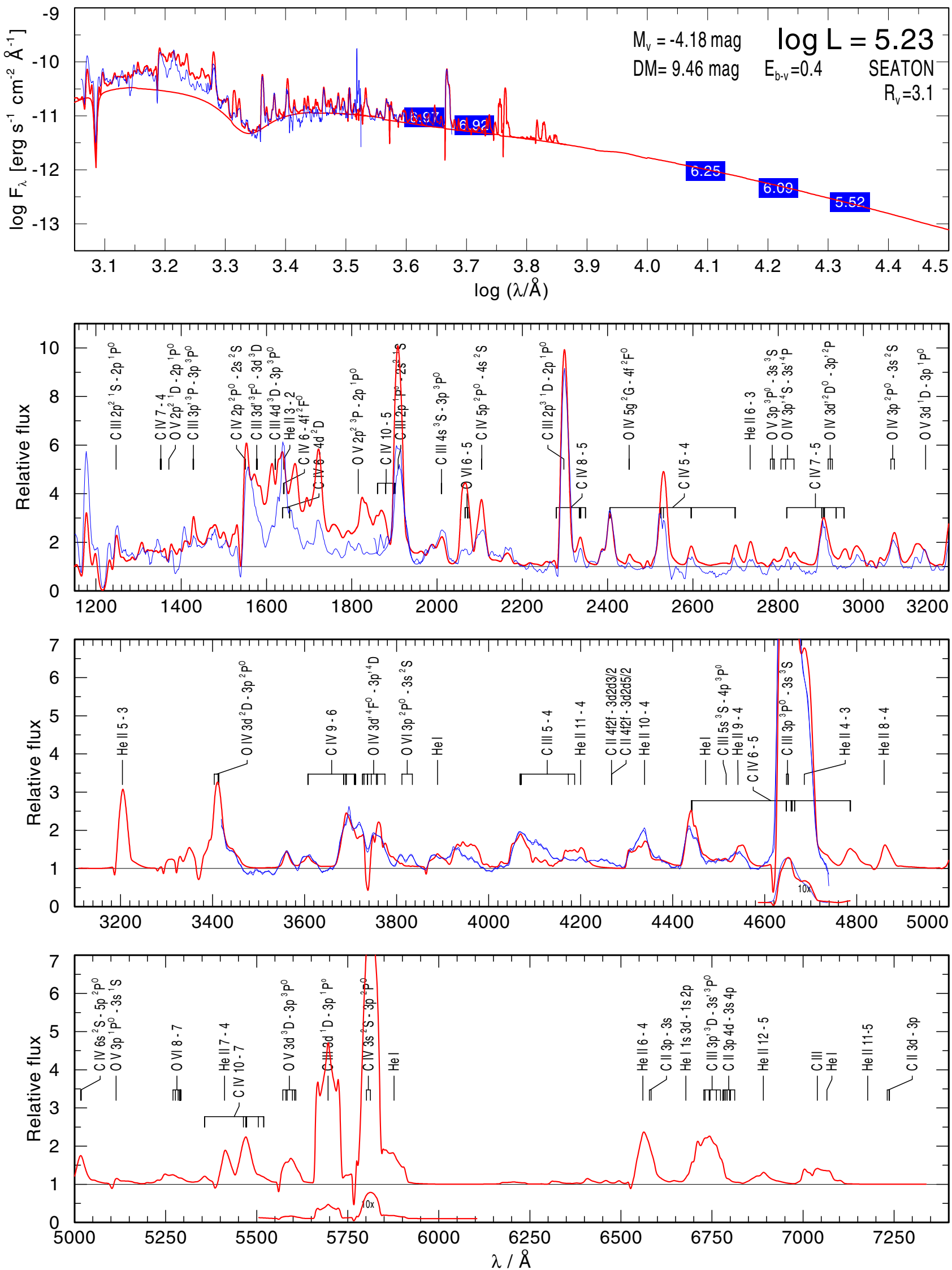

Fig. B.31. Spectral fit for WR 90. 
WR092

$\mathrm{T}_{*}=45 \mathrm{kK} \log \mathrm{R}_{\mathrm{t}}=0.8$
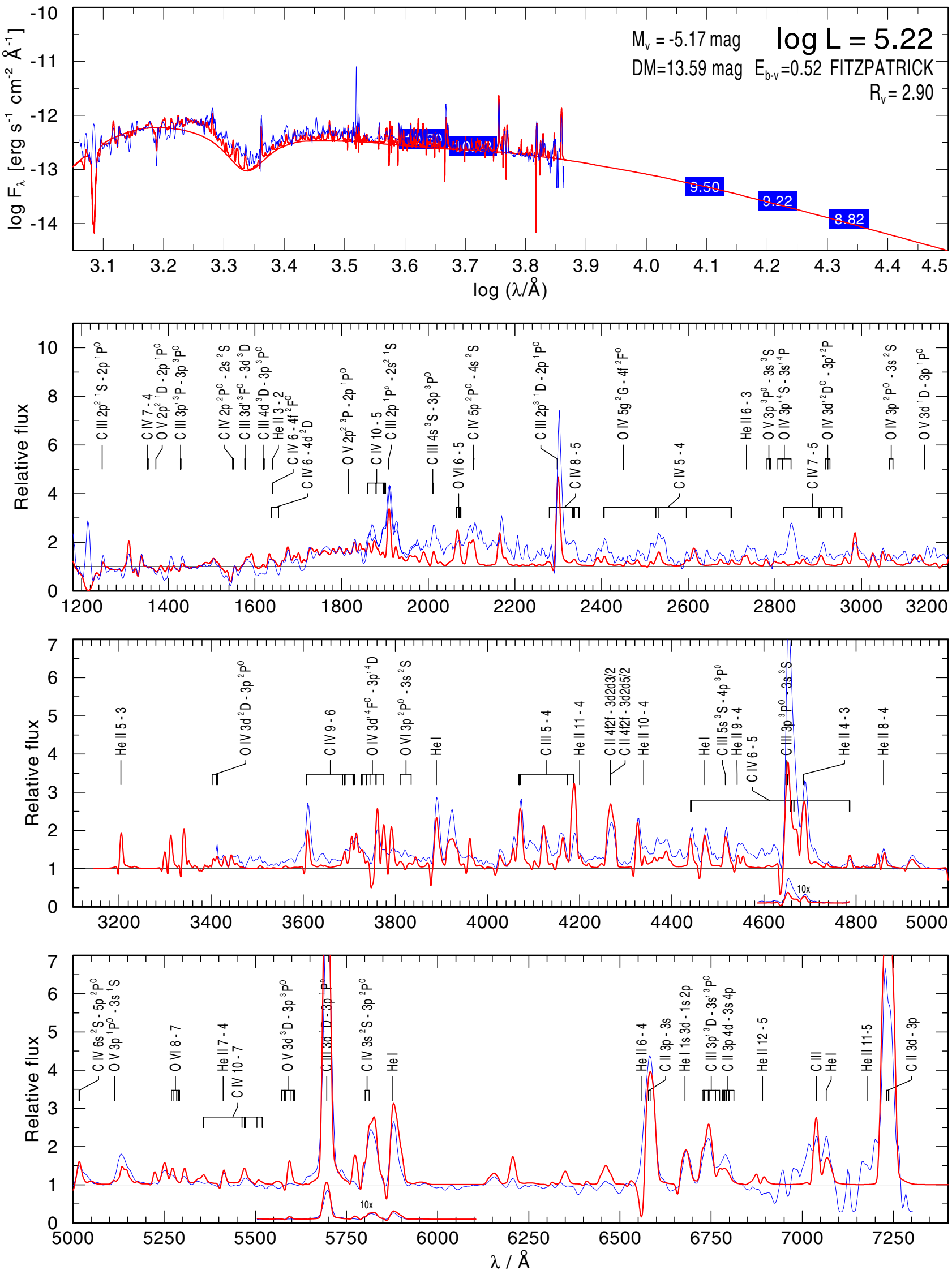

Fig. B.32. Spectral fit for WR 92. 
WR095

WC9d

$\mathrm{T}_{*}=45 \mathrm{kK} \log \mathrm{R}_{\mathrm{t}}=0.9$

$v_{\infty}=2000 \mathrm{~km} / \mathrm{s}-\mathrm{He}: \mathrm{C}: \mathrm{O}=55: 40: 5$
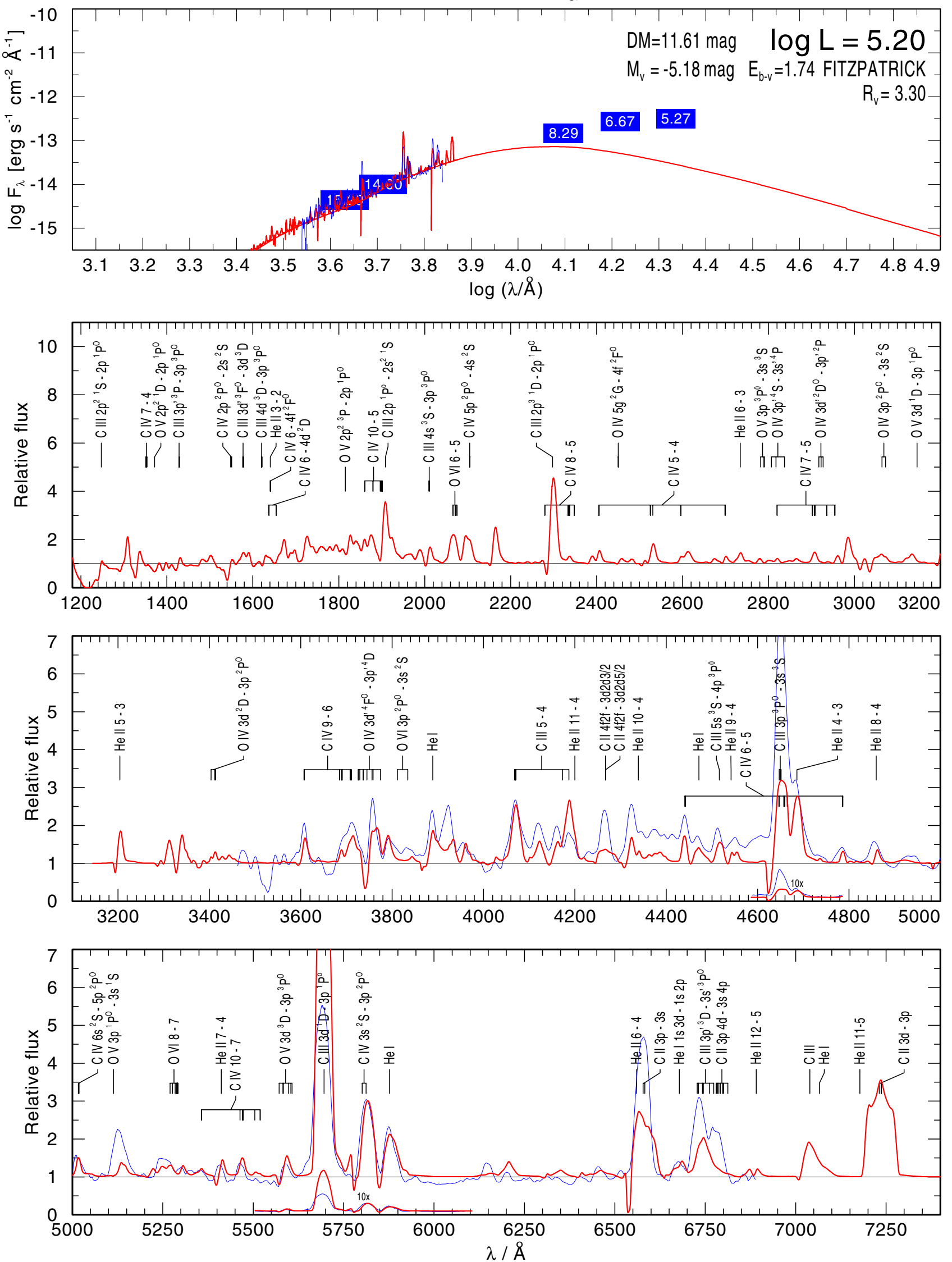

Fig. B.33. Spectral fit for WR 95. 
A. Sander et al.: The Galactic WC stars

WR098 (Binary)

$T_{*}=50 \mathrm{kK} \log R_{t}=1.0$

WN8/WC7

$\mathrm{v}_{\infty}=1600 \mathrm{~km} / \mathrm{s}-\mathrm{He}: \mathrm{C}: \mathrm{N}=93: 5: 1.5$
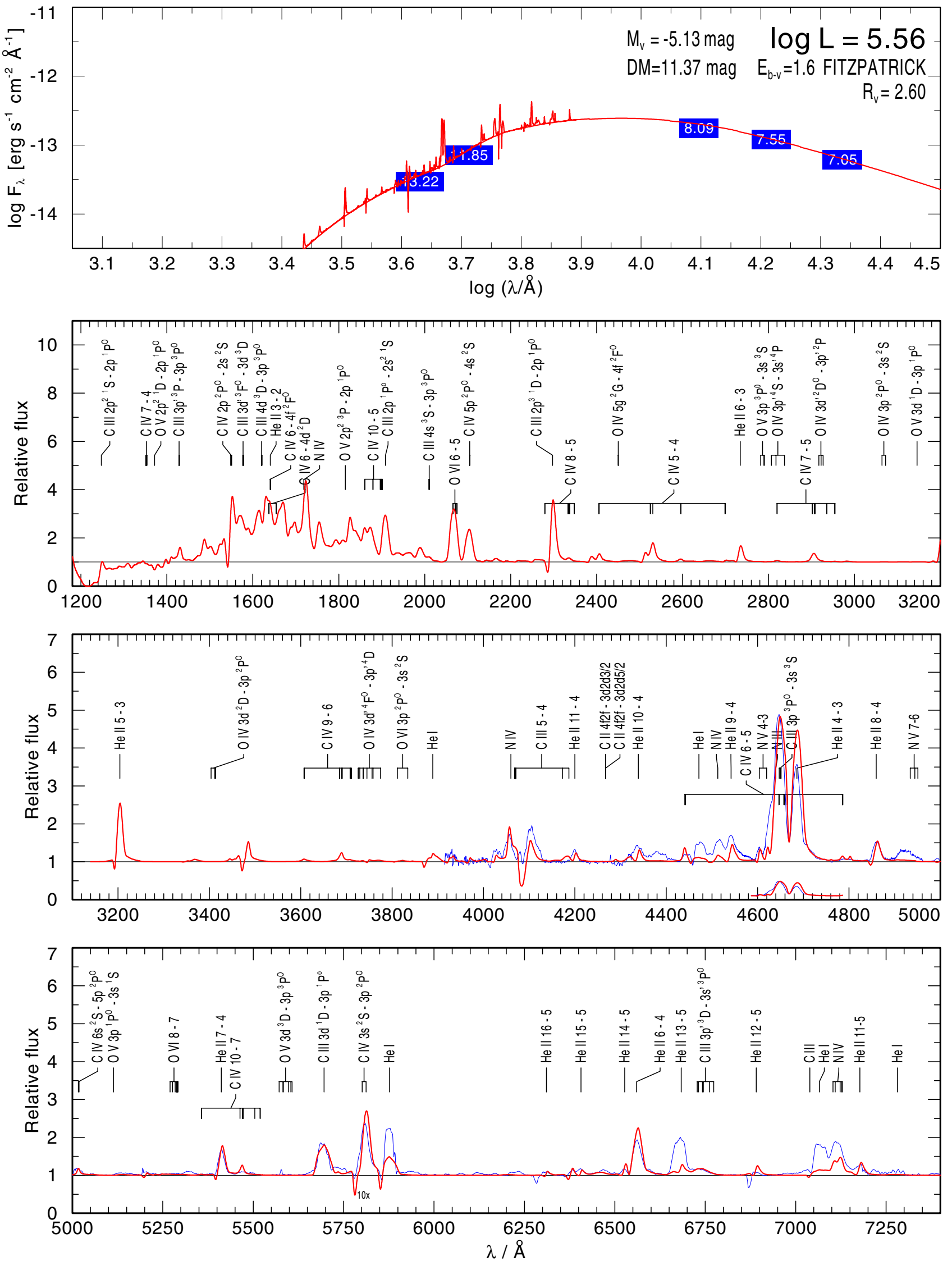

Fig. B.34. Spectral fit for WR 98. 
WR102

$T_{*}=200 \mathrm{kK} \log R_{t}=0.4$

WO2
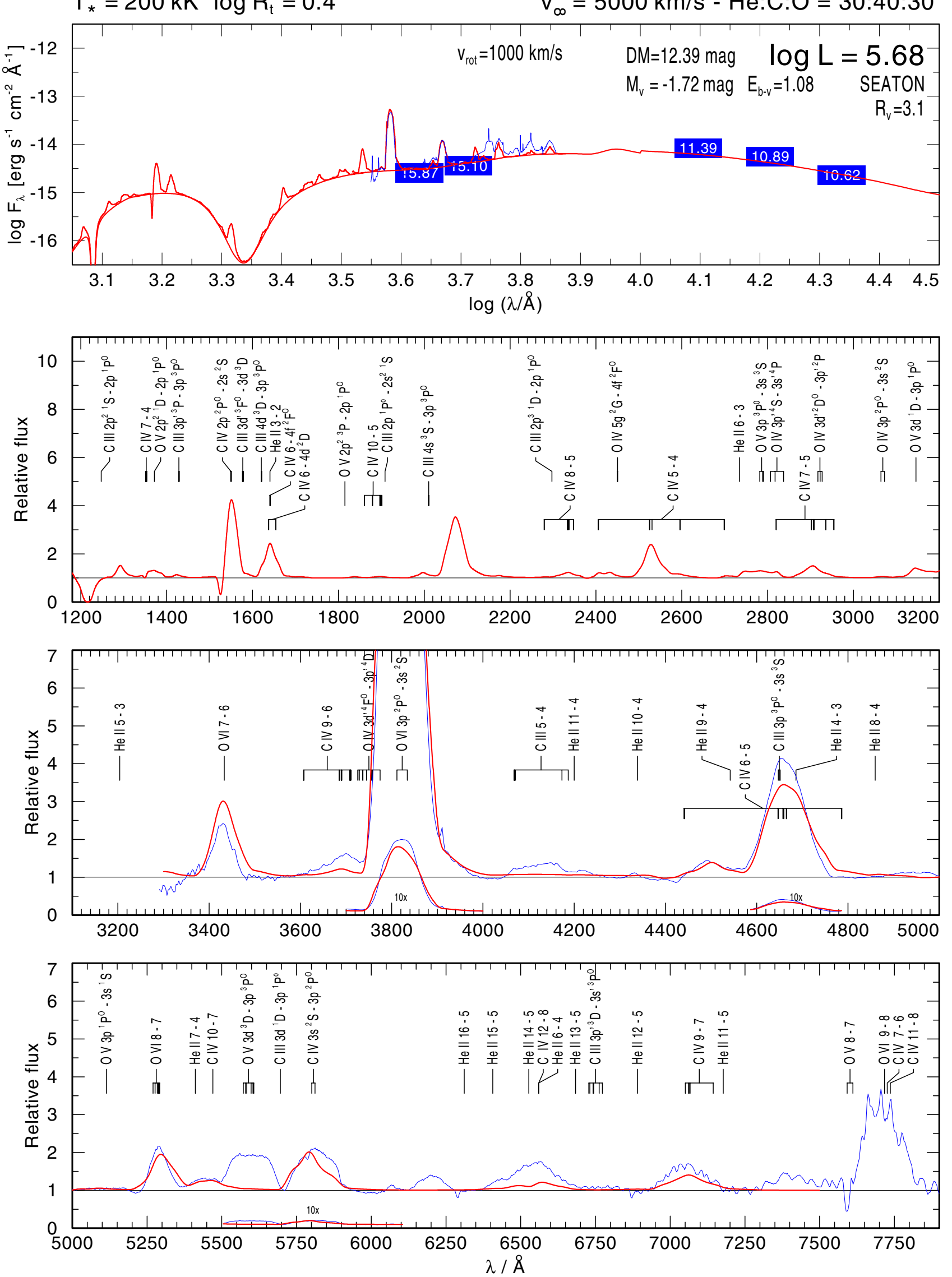

Fig. B.35. Spectral fit for WR 102 . 
A. Sander et al.: The Galactic WC stars

WR103

$T_{*}=45 \mathrm{kK} \log R_{t}=0.8$

WC9d

$v_{\infty}=1000 \mathrm{~km} / \mathrm{s}-\mathrm{He}: \mathrm{C}: \mathrm{O}=55: 40: 5$
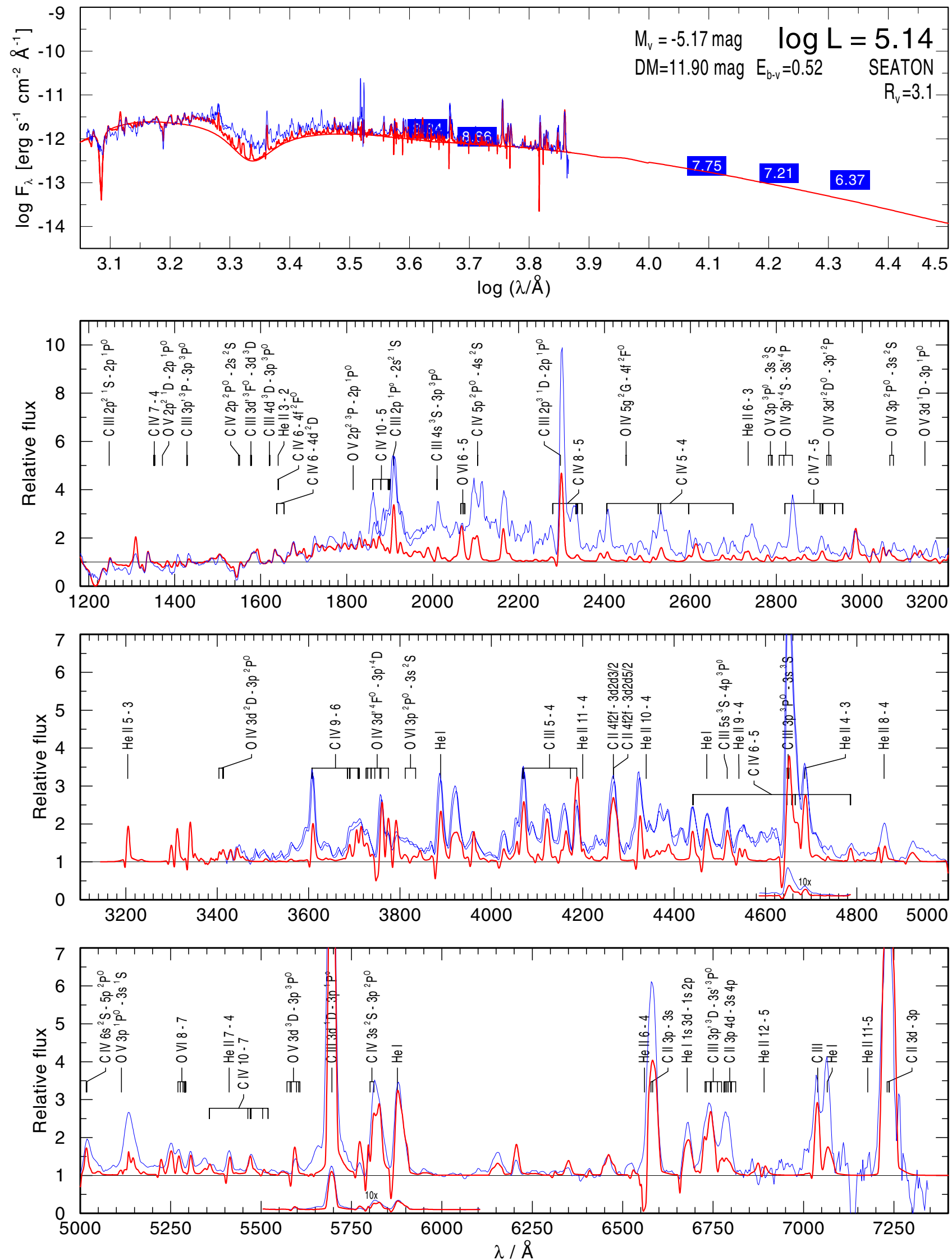

Fig. B.36. Spectral fit for WR 103. 
WR104 (Binary)

WC9d

$T_{*}=40 \mathrm{kK} \log R_{\mathrm{t}}=1.0$

$v_{\infty}=1600 \mathrm{~km} / \mathrm{s}-\mathrm{He}: \mathrm{C}: \mathrm{O}=55: 40: 5$
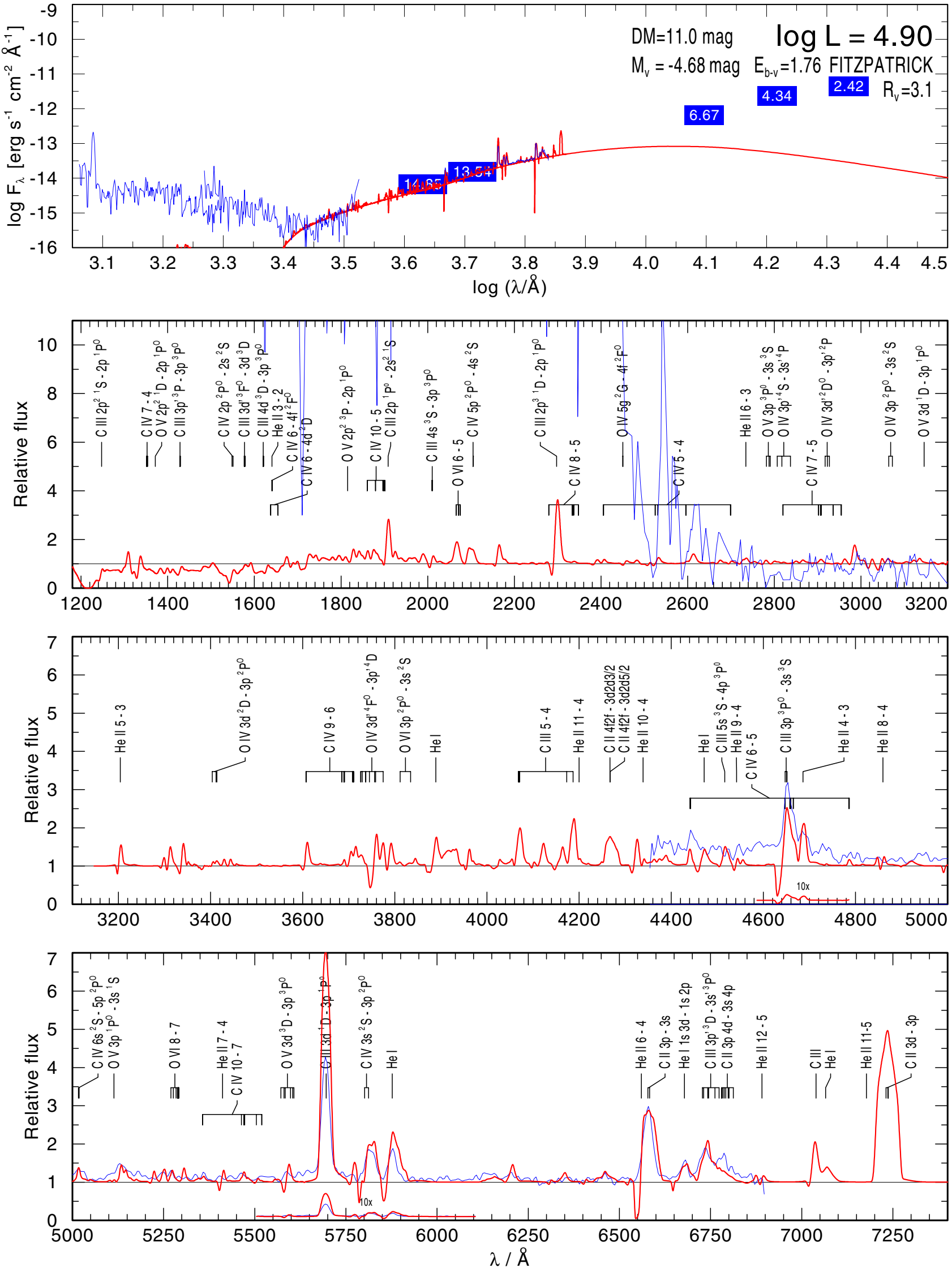

Fig. B.37. Spectral pseudo fit for WR 104. 
A. Sander et al.: The Galactic WC stars

WR106

$T_{*}=45 \mathrm{kK} \log R_{t}=0.8$

WC9d $v_{\infty}=1000 \mathrm{~km} / \mathrm{s}-\mathrm{He}: \mathrm{C}: \mathrm{O}=55: 40: 5$
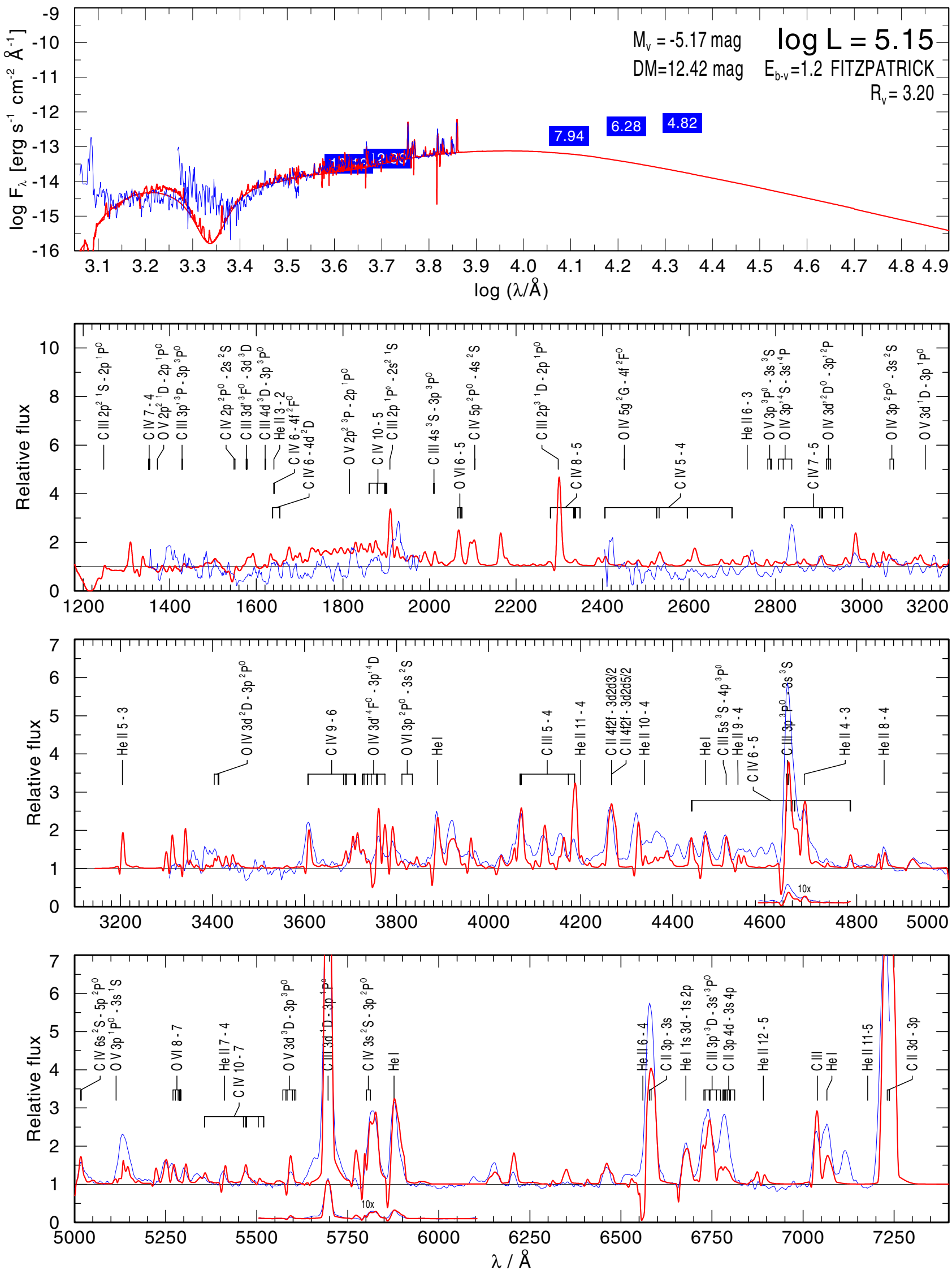

Fig. B.38. Spectral fit for WR 106. 
WR111

WC5

$T_{*}=89 \mathrm{kK} \log R_{\mathrm{t}}=0.4$

$v_{\infty}=2000 \mathrm{~km} / \mathrm{s}-\mathrm{He}: \mathrm{C}: \mathrm{O}=55: 40: 5$
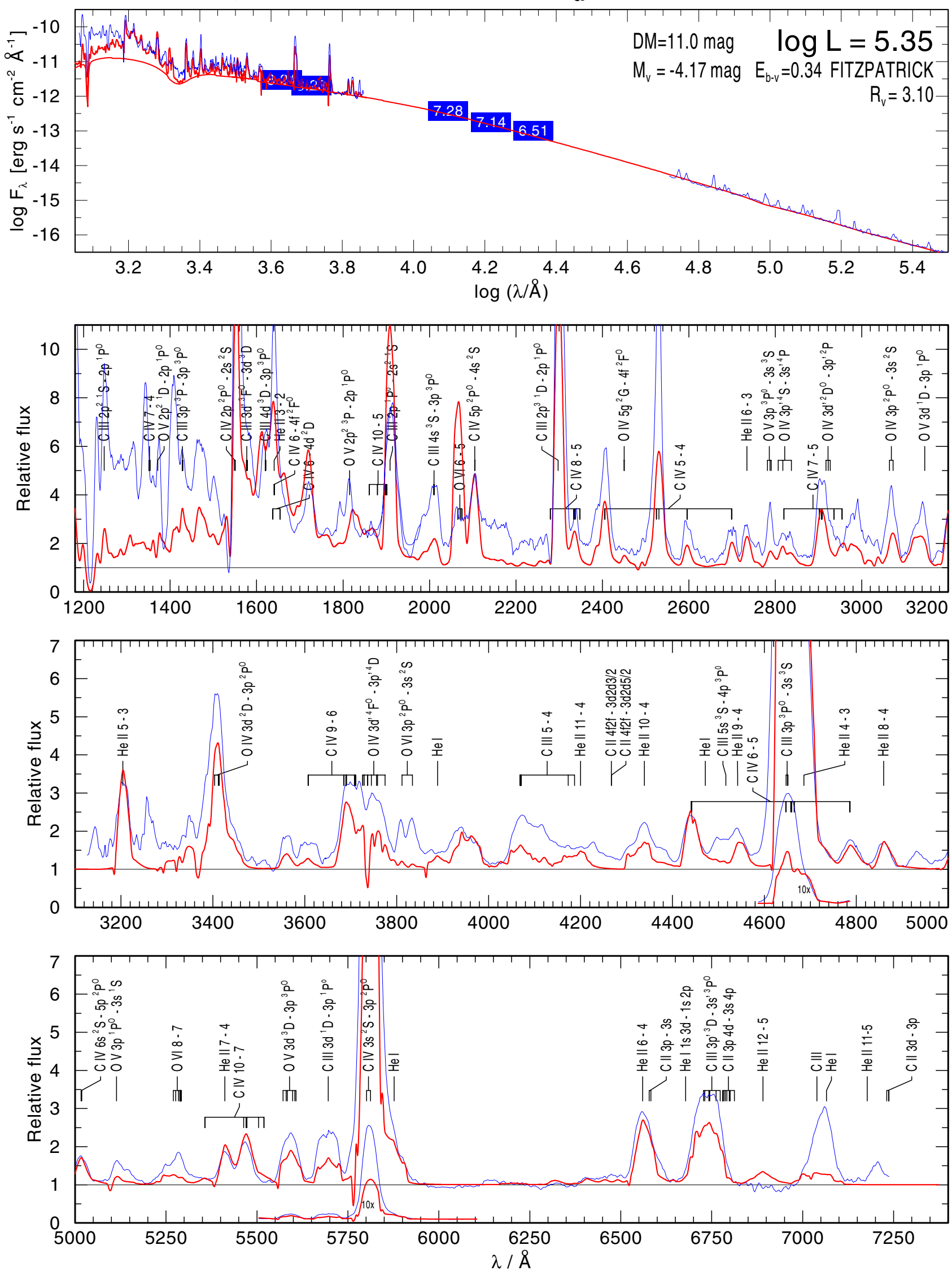

Fig. B.39. Spectral fit for WR 111 . 
A. Sander et al.: The Galactic WC stars

WR113 (Binary)

$T_{*}=50 \mathrm{kK} \log R_{t}=1.2$

WC8d

$v_{\infty}=2000 \mathrm{~km} / \mathrm{s}-\mathrm{He}: \mathrm{C}: \mathrm{O}=55: 40: 5$
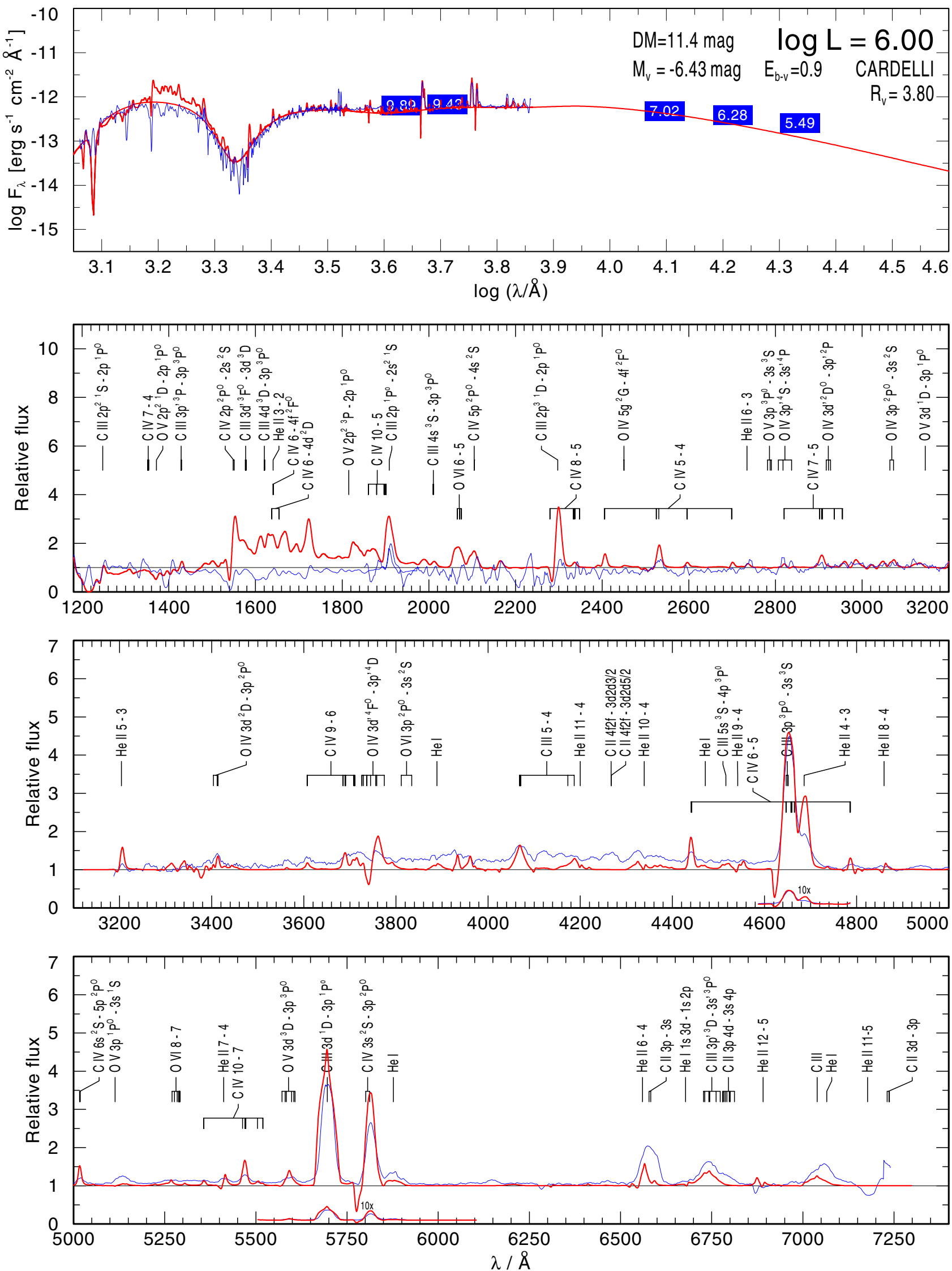

Fig. B.40. Spectral pseudo fit for WR 113. 
WR114

$\mathrm{T}_{*}=79 \mathrm{kK} \log \mathrm{R}_{\mathrm{t}}=0.5$

WC5

$\mathrm{v}_{\infty}=2000 \mathrm{~km} / \mathrm{s}-\mathrm{He}: \mathrm{C}: \mathrm{O}=55: 40: 5$
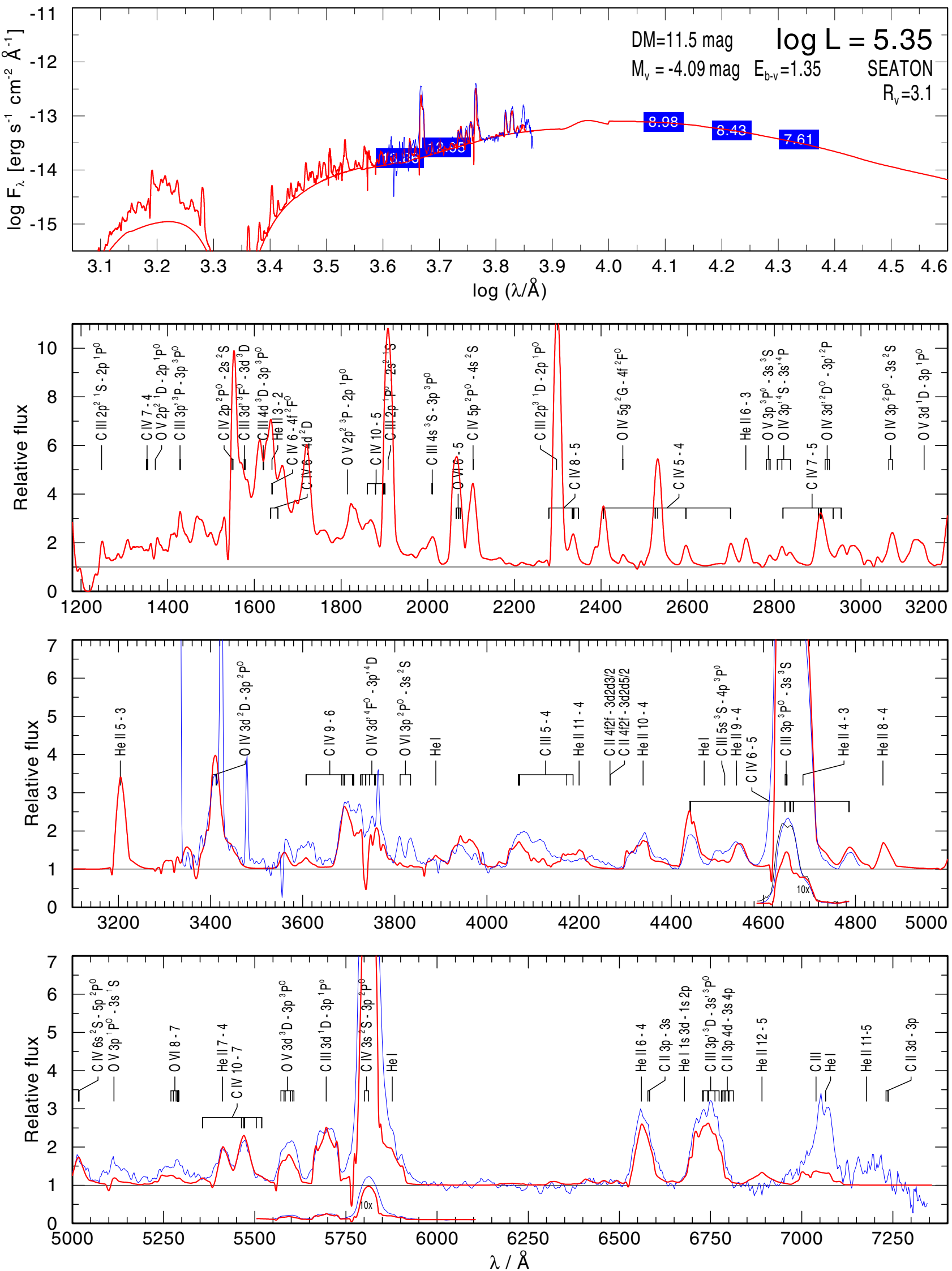

Fig. B.41. Spectral fit for WR 114 . 
WR117

$\mathrm{T}_{*}=56 \mathrm{kK} \log \mathrm{R}_{\mathrm{t}}=0.6$
WC9d $v_{\infty}=2000 \mathrm{~km} / \mathrm{s}-\mathrm{He}: \mathrm{C}: \mathrm{O}=55: 40: 5$
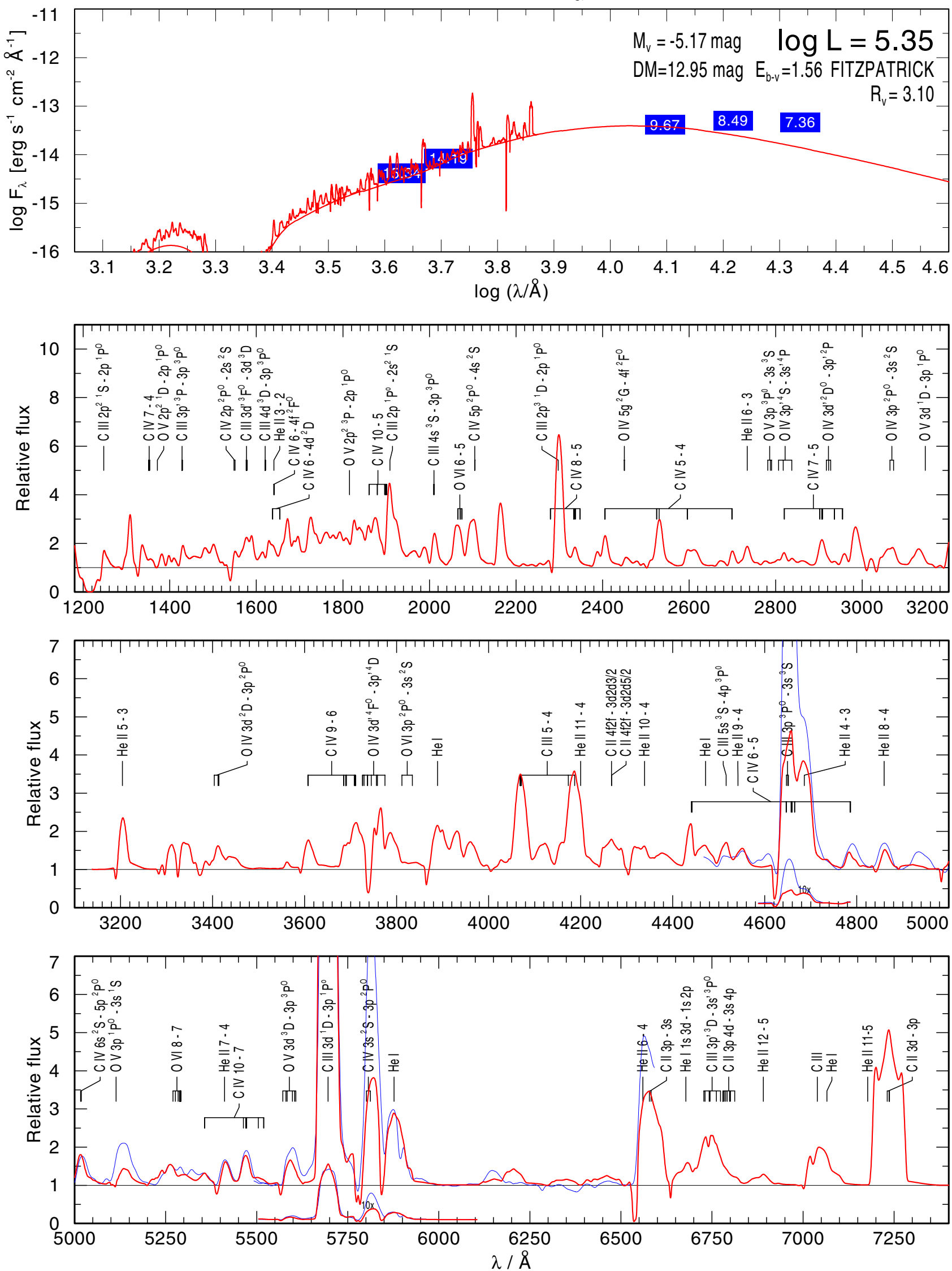

Fig. B.42. Spectral fit for WR 117 . 
WR119

WC9d

$\mathrm{T}_{*}=45 \mathrm{kK} \log \mathrm{R}_{\mathrm{t}}=0.8$

$v_{\infty}=1000 \mathrm{~km} / \mathrm{s}-\mathrm{He}: \mathrm{C}: \mathrm{O}=55: 40: 5$
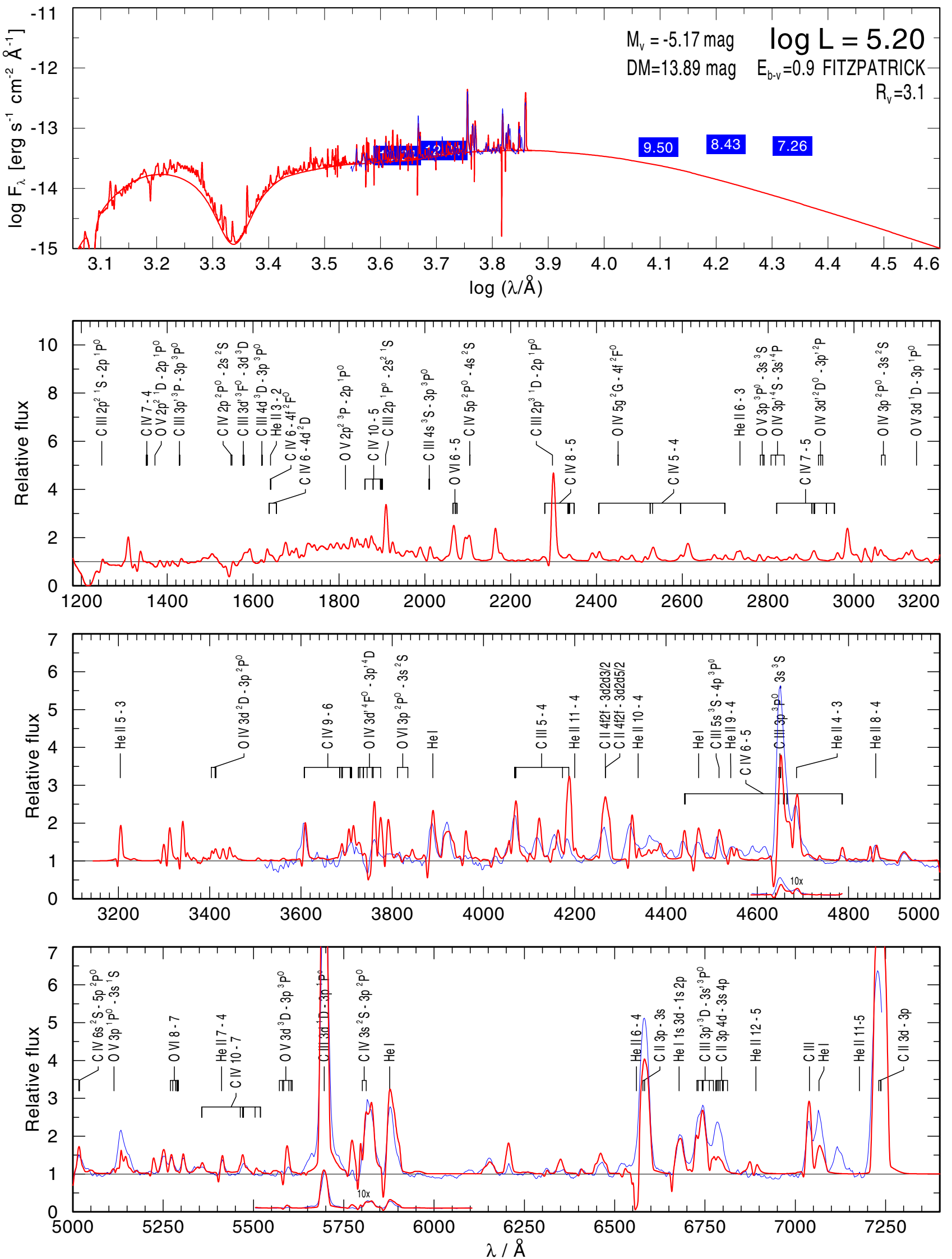

Fig. B.43. Spectral fit for WR 119. 
A. Sander et al.: The Galactic WC stars

WR121

WC9d

$\mathrm{T}_{*}=45 \mathrm{kK} \log \mathrm{R}_{\mathrm{t}}=0.8$

$v_{\infty}=1000 \mathrm{~km} / \mathrm{s}-\mathrm{He}: \mathrm{C}: \mathrm{O}=55: 40: 5$
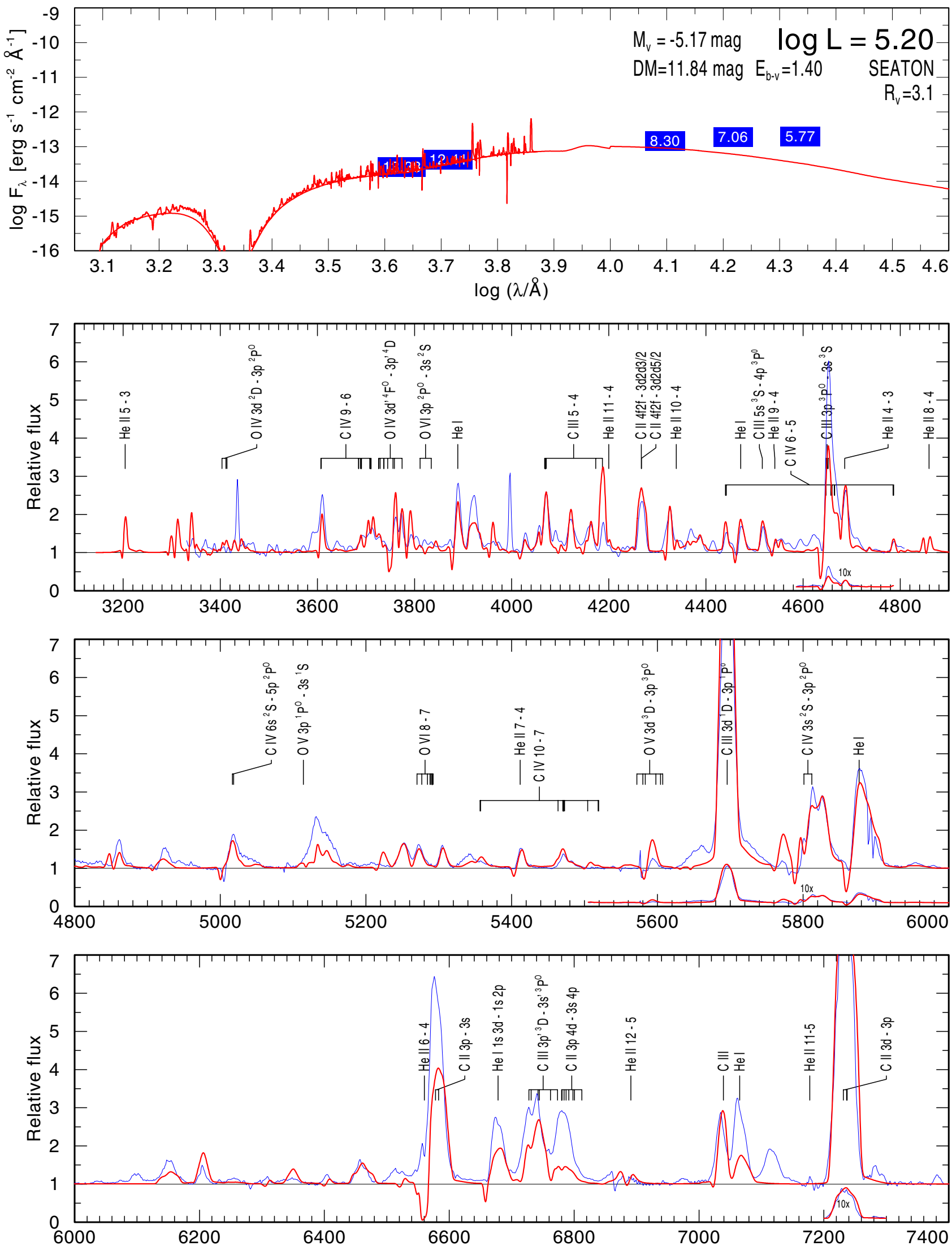

Fig. B.44. Spectral fit for WR 121 . 
WR125 (Binary)
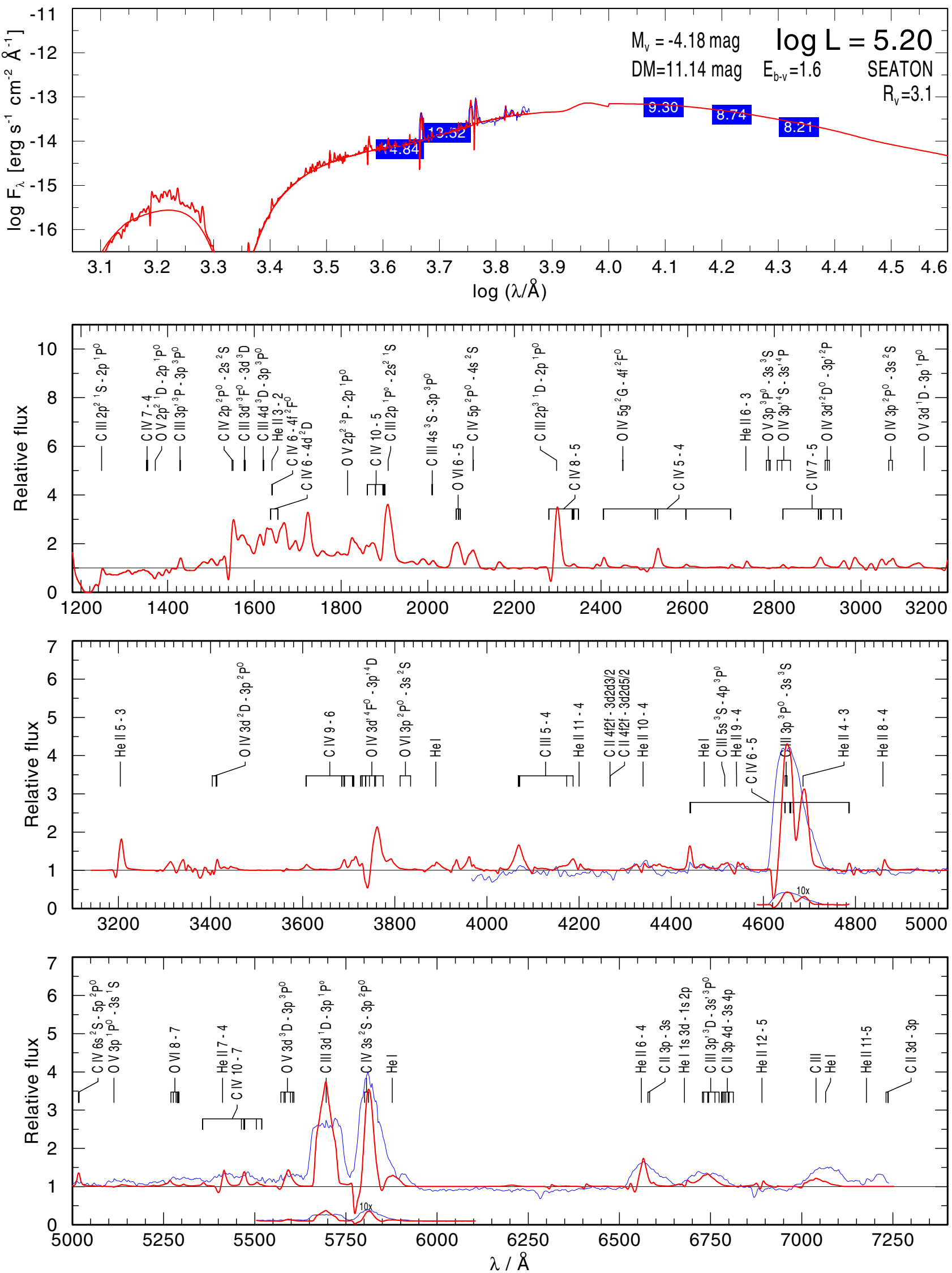

Fig. B.45. Spectral pseudo fit for WR 125 . 
WR126

$\mathrm{WC} 5 / \mathrm{WN}$

$T_{*}=63 \mathrm{kK} \log R_{t}=1.2$

$v_{\infty}=2000 \mathrm{~km} / \mathrm{s}-\mathrm{He}: \mathrm{C}: \mathrm{O}=75: 20: 5$
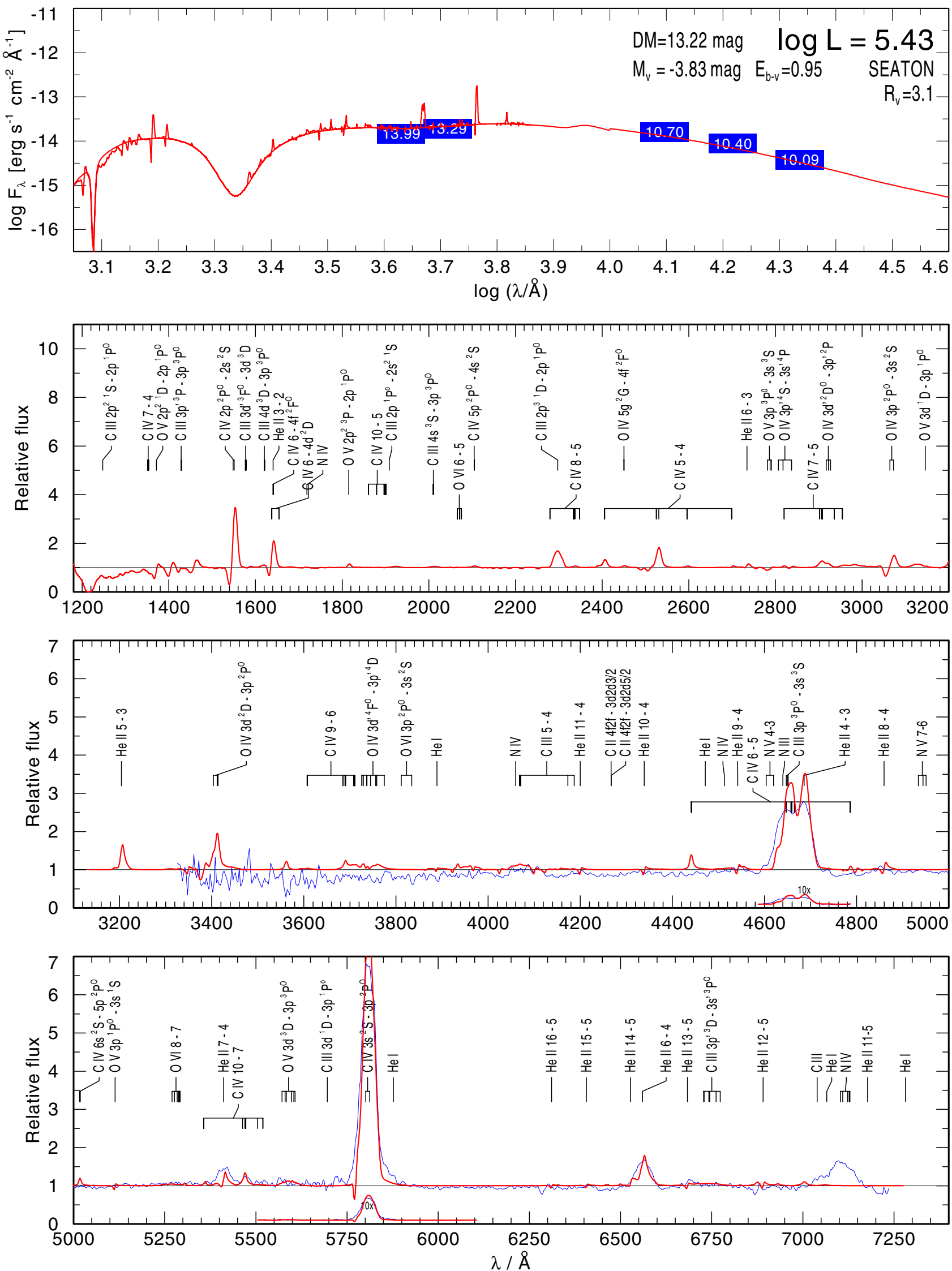

Fig. B.46. Spectral fit for WR 126. 
WR132

$T_{*}=71 \mathrm{kK} \log R_{t}=0.6$

WC6

$\mathrm{v}_{\infty}=2000 \mathrm{~km} / \mathrm{s}-\mathrm{He}: \mathrm{C}: \mathrm{O}=55: 40: 5$
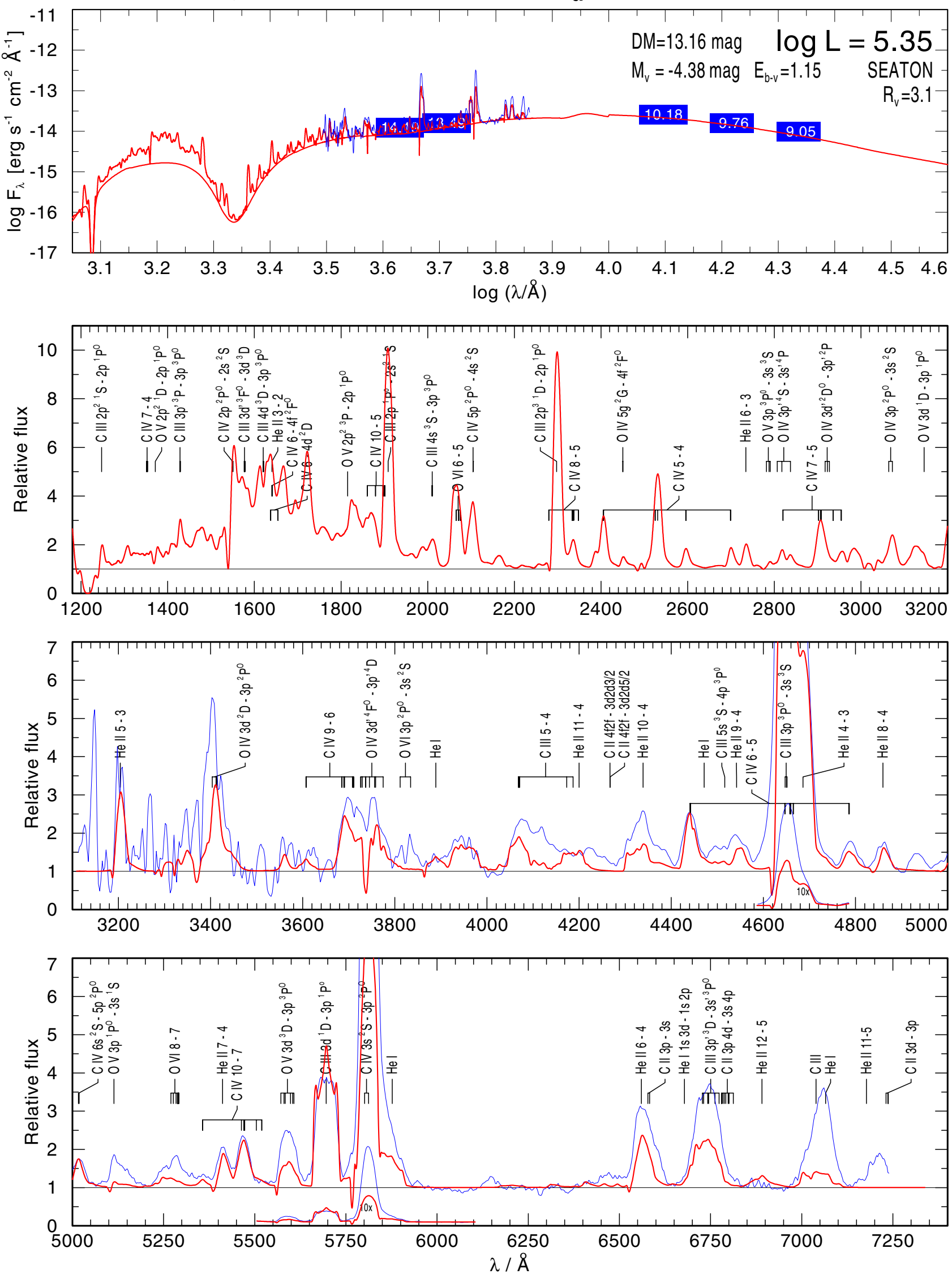

Fig. B.47. Spectral fit for WR 132. 
WR135

WC8

$T_{*}=63 \mathrm{kK} \log R_{t}=0.6$

$v_{\infty}=2000 \mathrm{~km} / \mathrm{s}-\mathrm{He}: \mathrm{C}: \mathrm{O}=75: 20: 5$
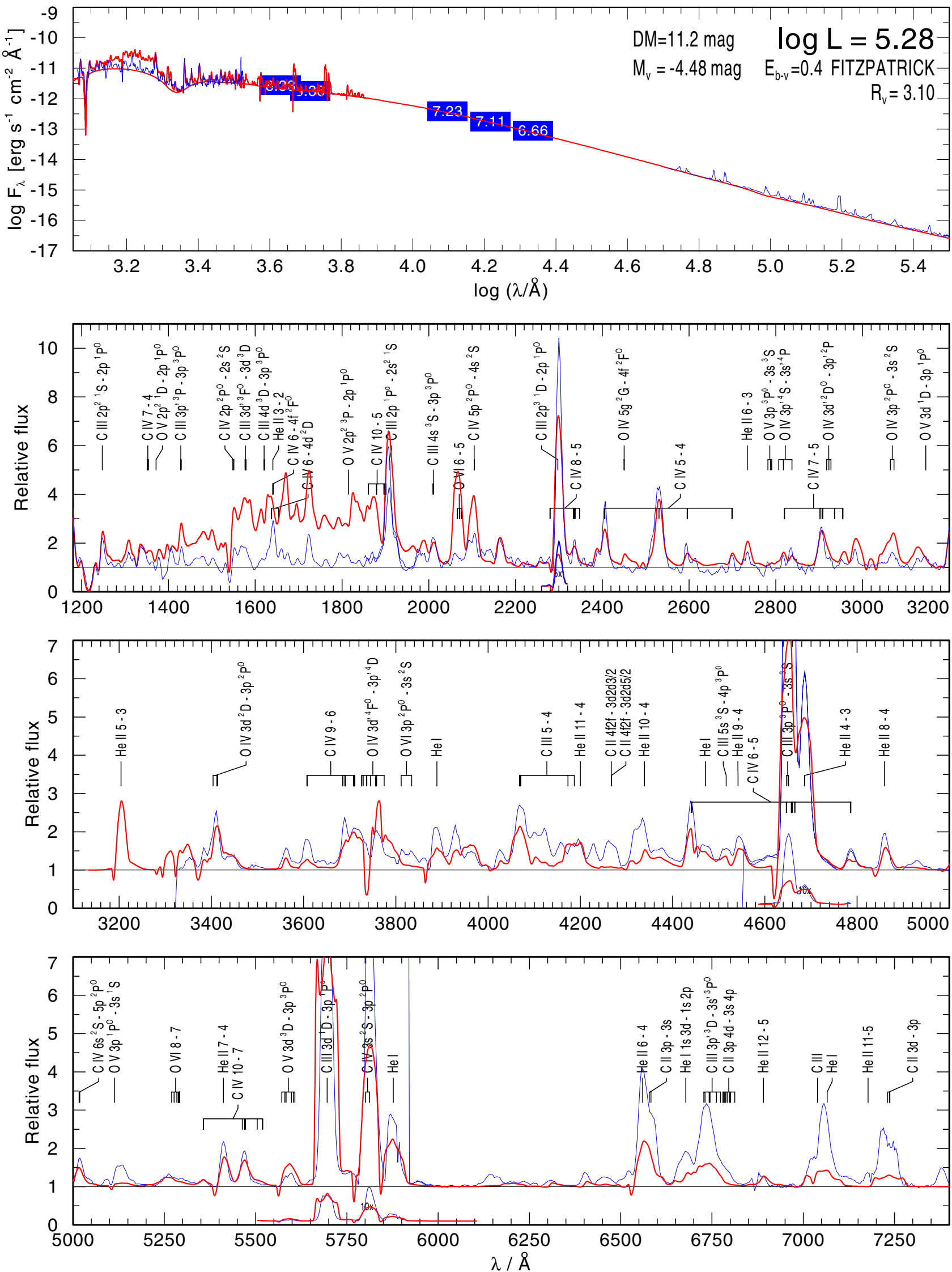

Fig. B.48. Spectral fit for WR 135 . 
WR137 (Binary)

$\mathrm{T}_{*}=56 \mathrm{kK} \log \mathrm{R}_{\mathrm{t}}=1.0$
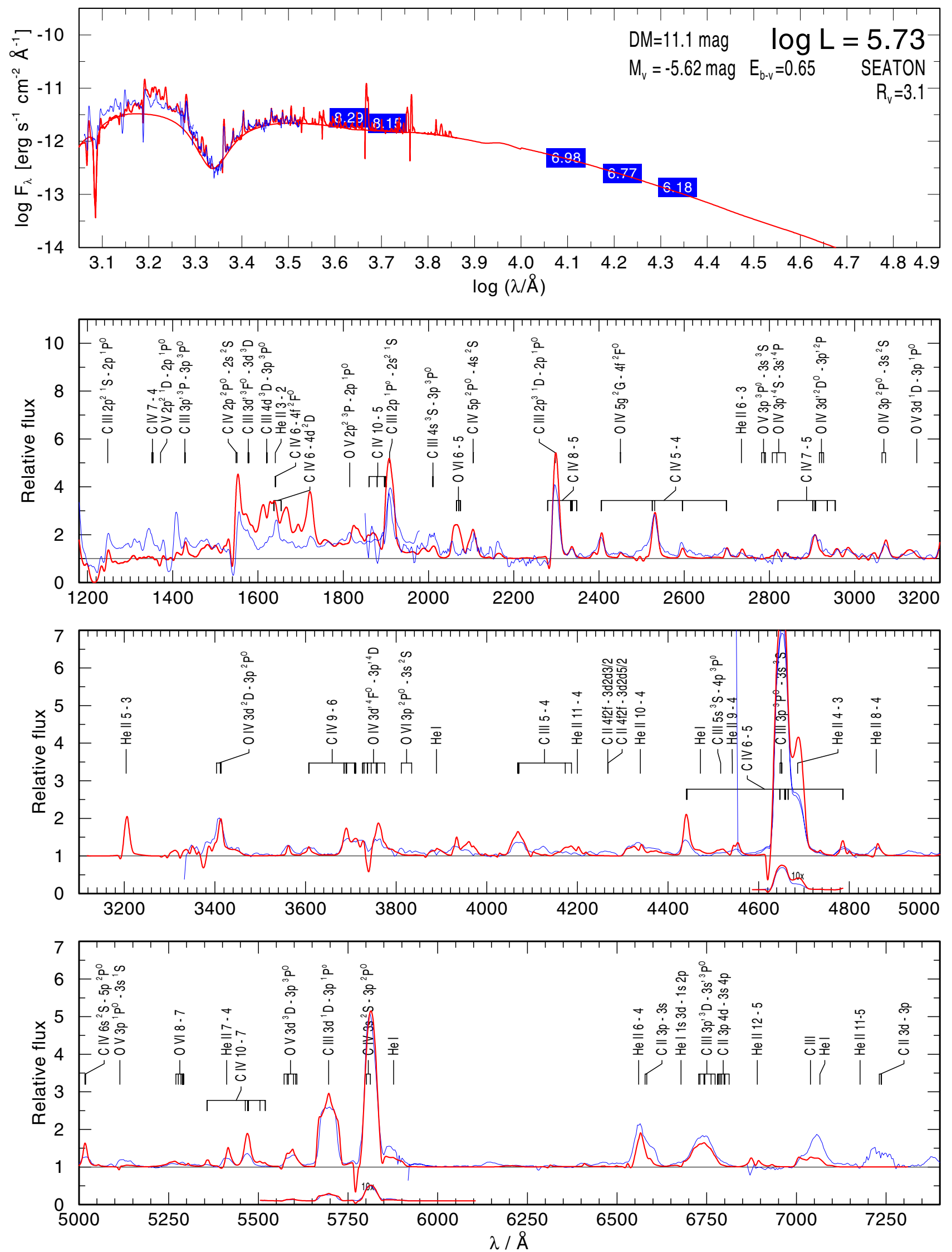

Fig. B.49. Spectral pseudo fit for WR 137. 
A. Sander et al.: The Galactic WC stars

WR142

$\mathrm{T}_{*}=200 \mathrm{kK} \log R_{\mathrm{t}}=0.3$

WO2
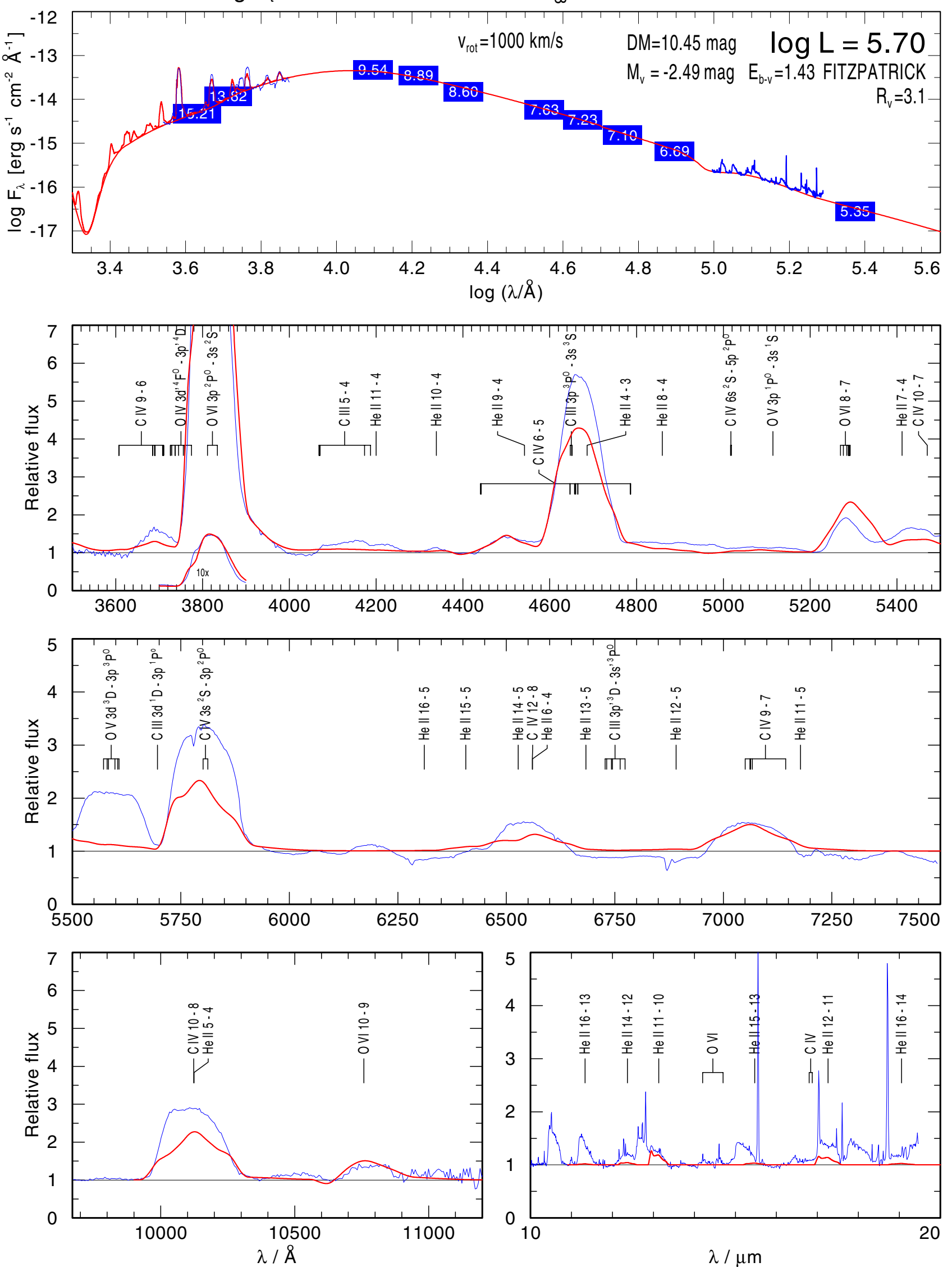

Fig. B.50. Spectral fit for WR 142 . 
WR143 (Binary)
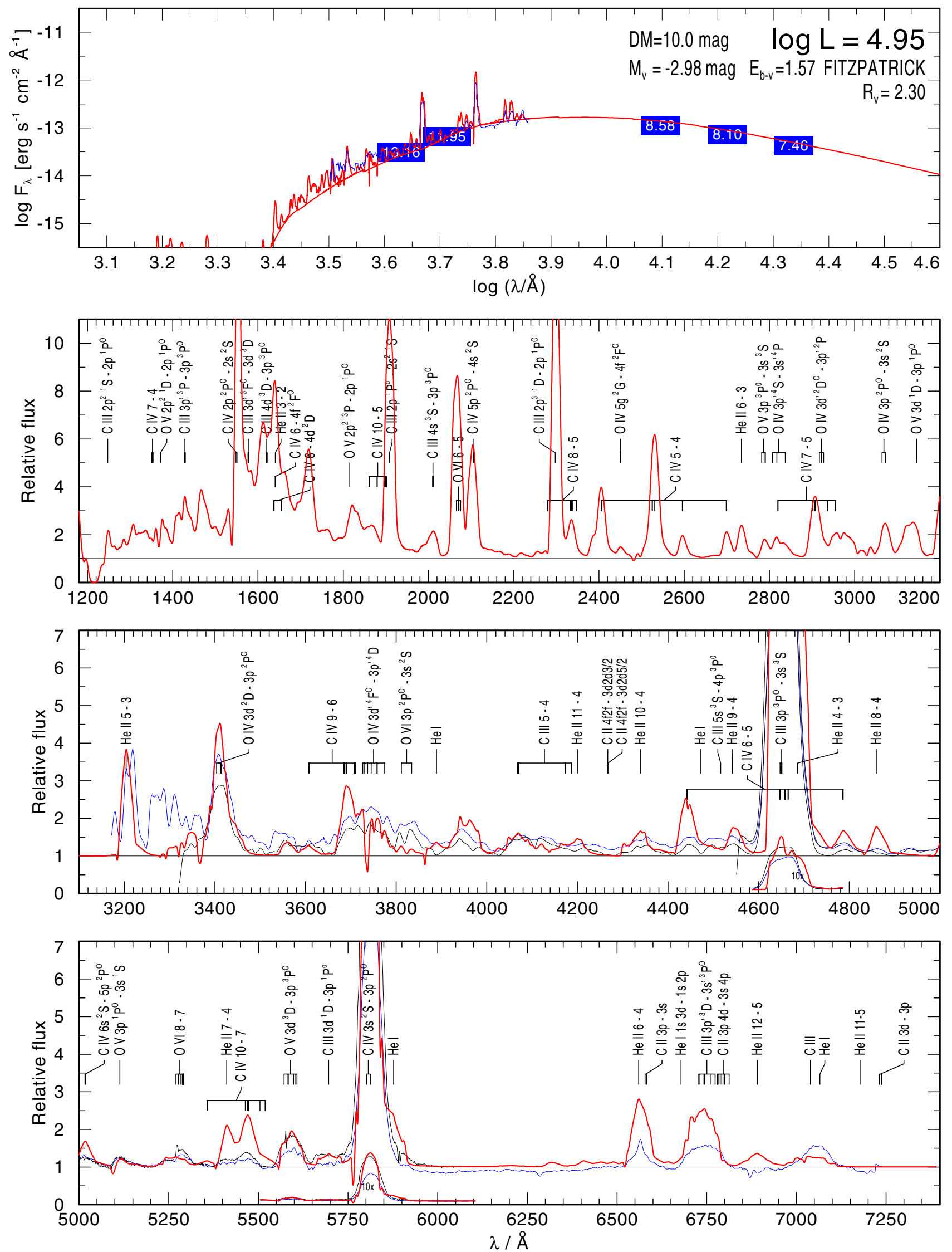

Fig. B.51. Spectral pseudo fit for WR 143 . 
A. Sander et al.: The Galactic WC stars

WR144

$T_{*}=112 \mathrm{kK} \log R_{\mathrm{t}}=0.2$

WC4

$\mathrm{v}_{\infty}=2000 \mathrm{~km} / \mathrm{s}-\mathrm{He}: \mathrm{C}: \mathrm{O}=35: 50: 15$
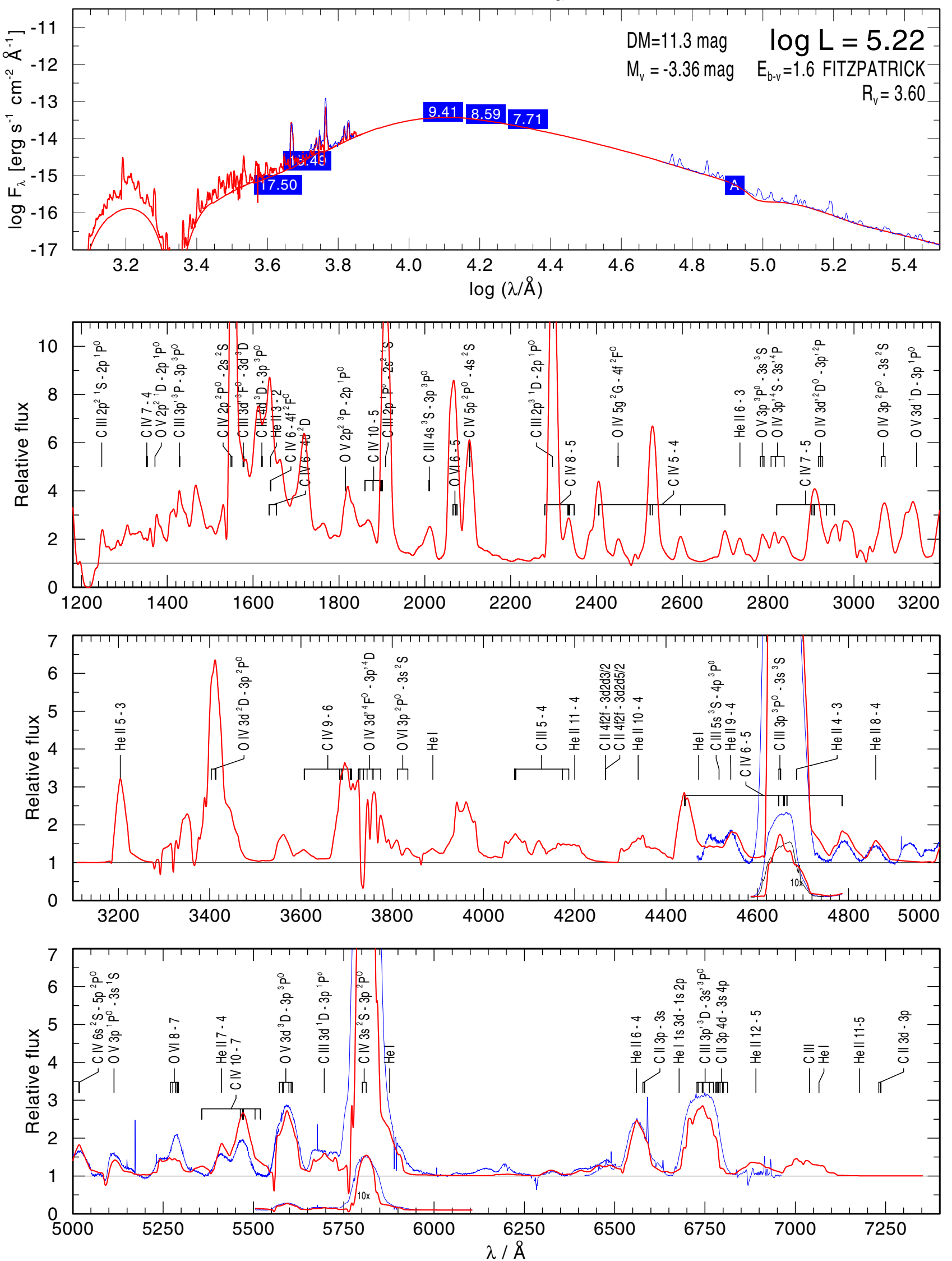

Fig. B.52. Spectral fit for WR 144 . 
WR145

WN7/WCE

$\mathrm{T}_{*}=50 \mathrm{kK} \log \mathrm{R}_{\mathrm{t}}=0.9$

$v_{\infty}=1600 \mathrm{~km} / \mathrm{s}-\mathrm{He}: \mathrm{C}: \mathrm{N}=98: 0.5: 1.5$
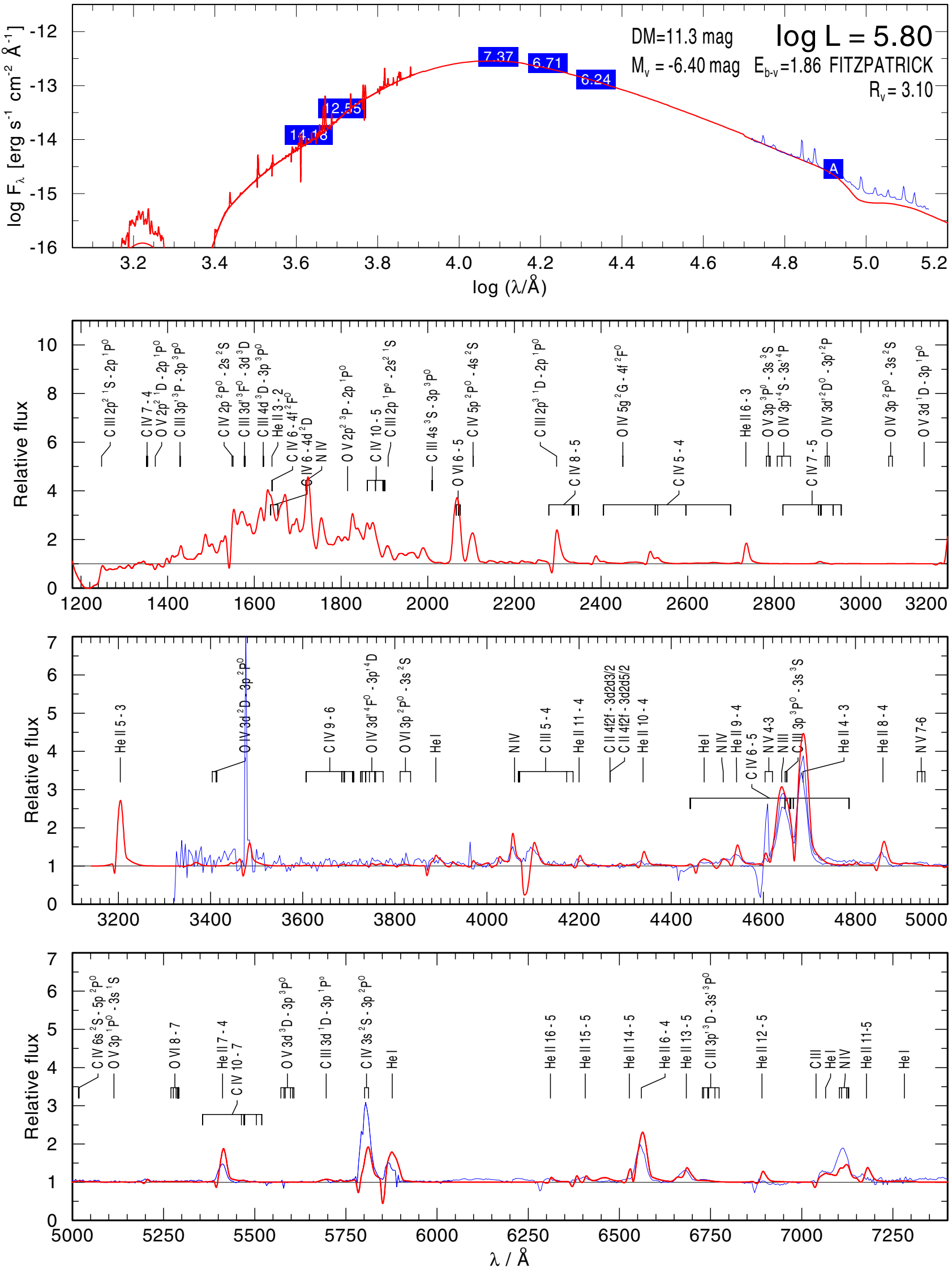

Fig. B.53. Spectral fit for WR 145 . 
A. Sander et al.: The Galactic WC stars

WR146 (Binary)

$\mathrm{T}_{*}=56 \mathrm{kK} \log R_{\mathrm{t}}=1.1$

WC5

$v_{\infty}=2000 \mathrm{~km} / \mathrm{s}-\mathrm{He}: \mathrm{C}: \mathrm{O}=35: 50: 15$
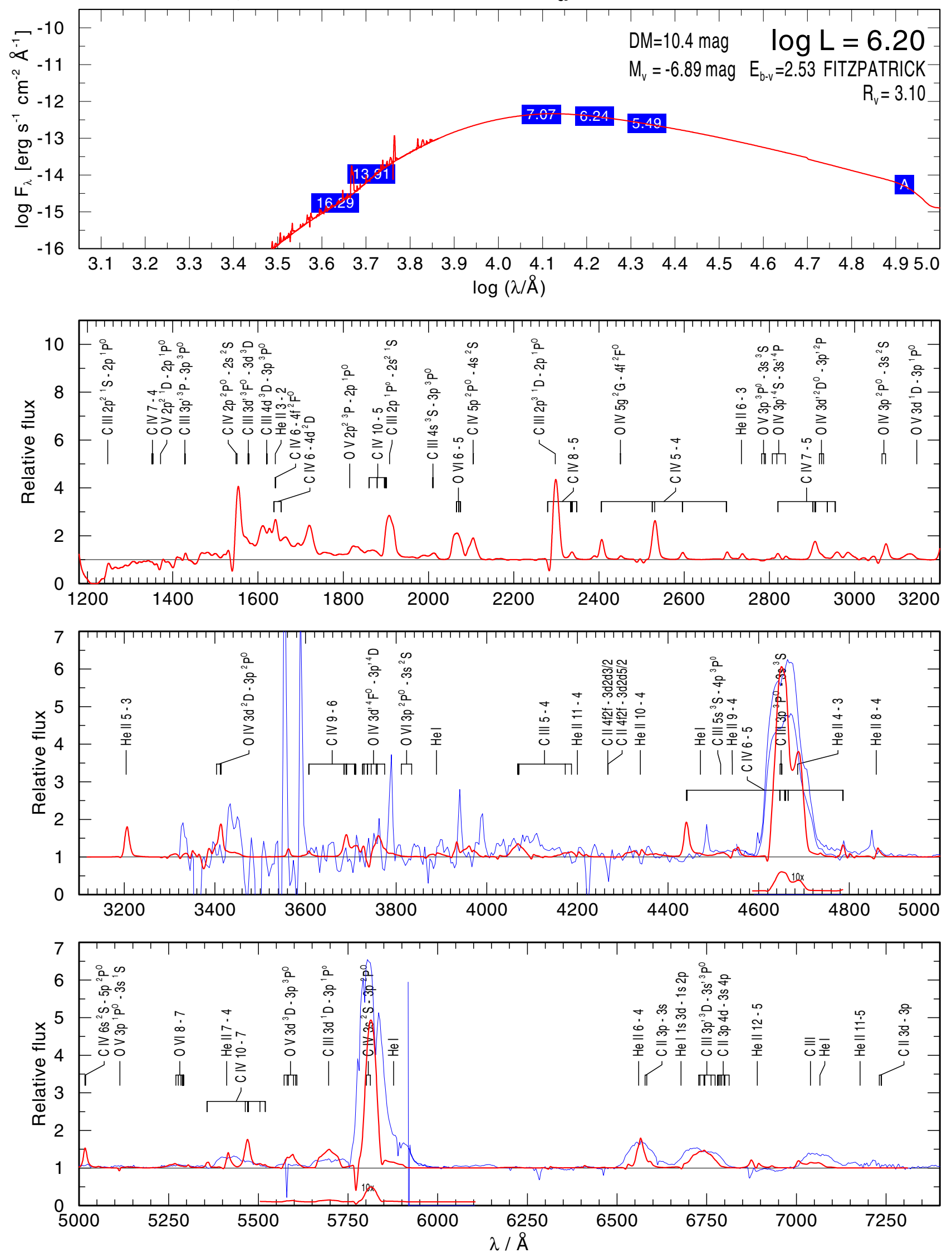

Fig. B.54. Spectral pseudo fit for WR 146. 
WR150

WC5

$\mathrm{T}_{*}=89 \mathrm{kK} \log \mathrm{R}_{\mathrm{t}}=0.4$

$v_{\infty}=2000 \mathrm{~km} / \mathrm{s}-\mathrm{He}: \mathrm{C}: \mathrm{O}=55: 40: 5$
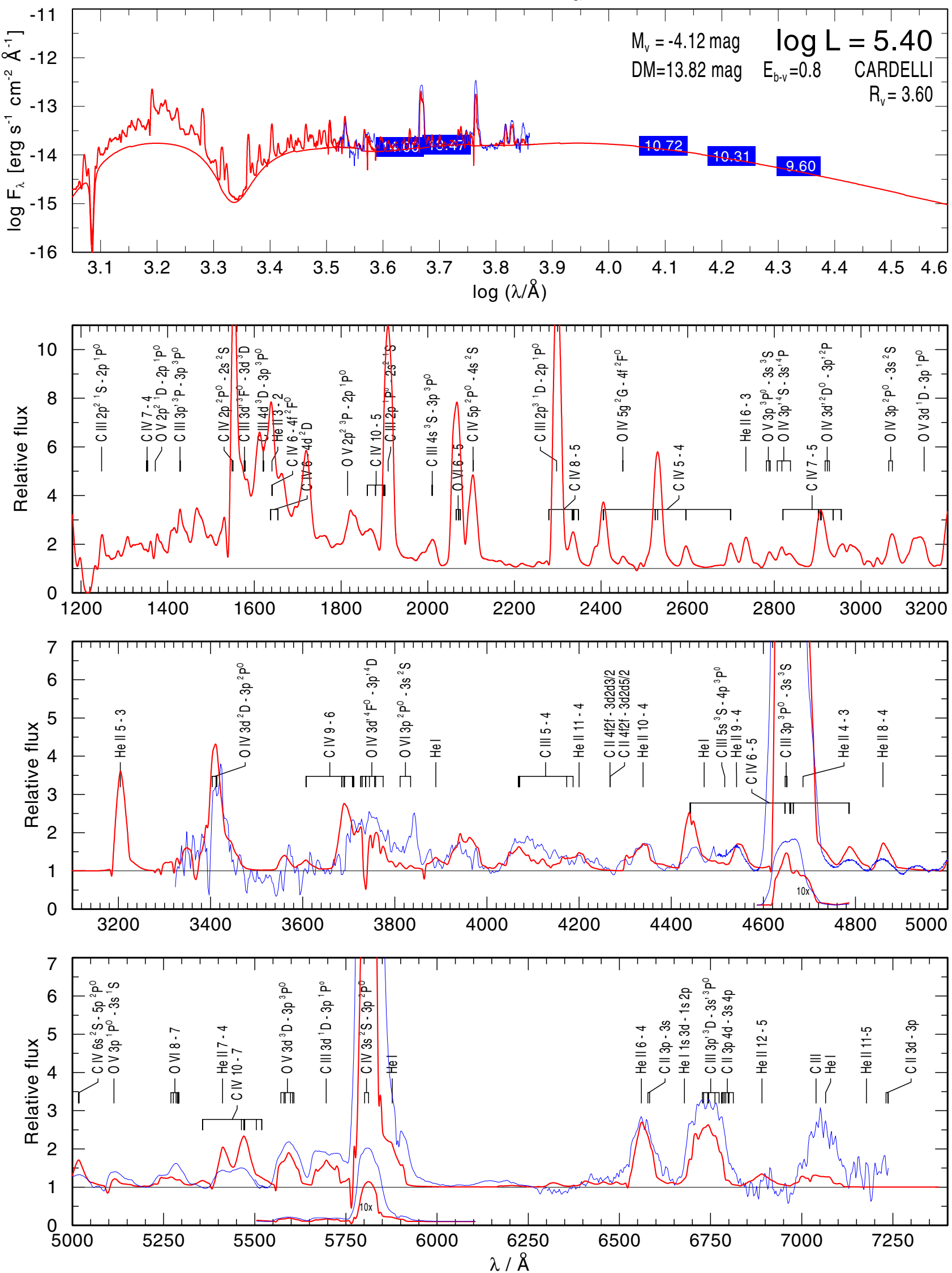

Fig. B.55. Spectral fit for WR 150 . 
A. Sander et al.: The Galactic WC stars

WR154

$\mathrm{T}_{*}=79 \mathrm{kK} \log \mathrm{R}_{\mathrm{t}}=0.5$

WC6

$v_{\infty}=2000 \mathrm{~km} / \mathrm{s}-\mathrm{He}: \mathrm{C}: \mathrm{O}=55: 40: 5$
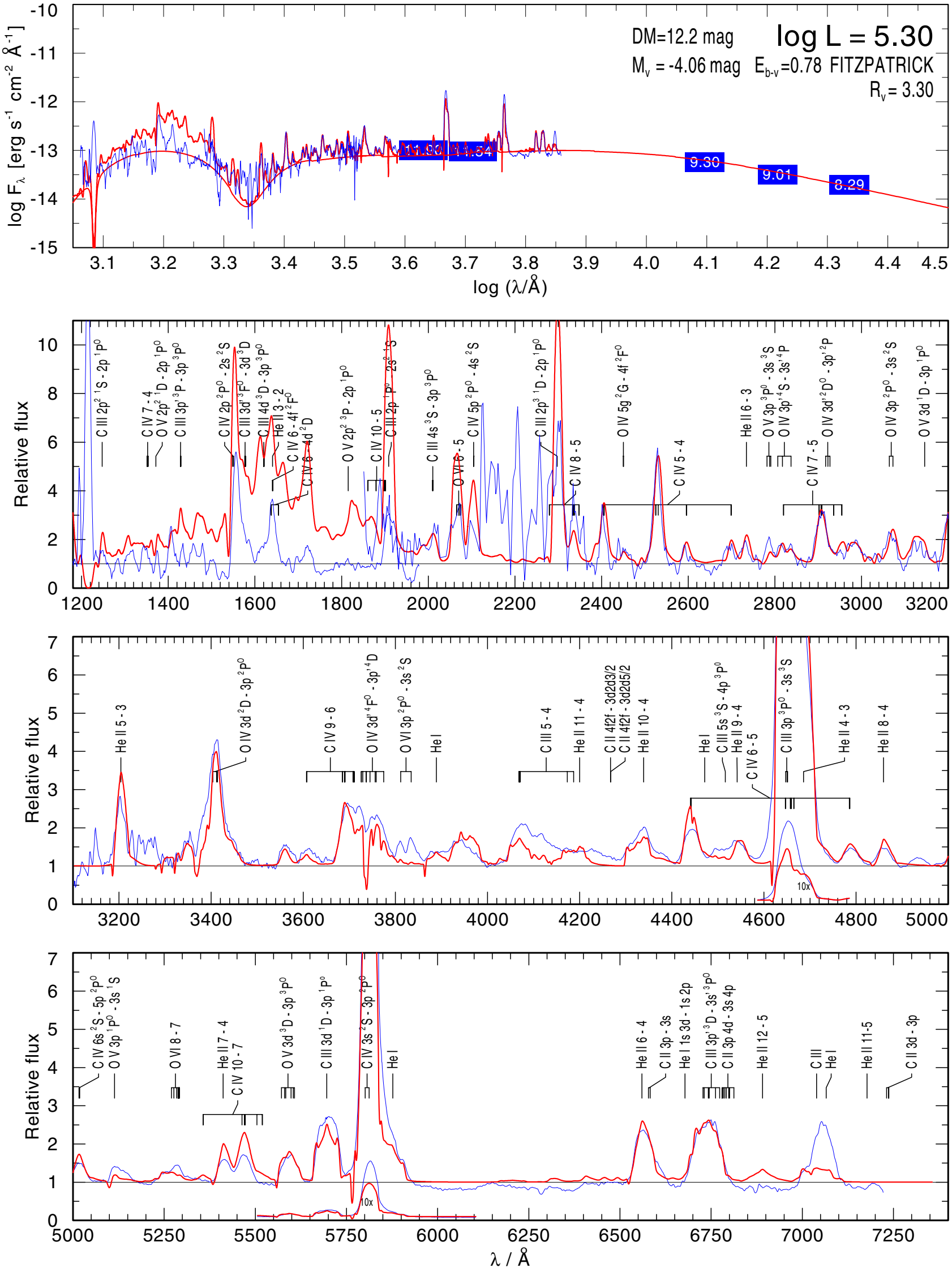

Fig. B.56. Spectral fit for WR 154 . 